\title{
WestVirginiaUniversity
}

THE RESEARCH REPOSITORY @ WVU

Graduate Theses, Dissertations, and Problem Reports

2005

\section{Actin nanokinematics under the influence of DC electric fields}

\author{
Raghu Chilakamarri \\ West Virginia University
}

Follow this and additional works at: https://researchrepository.wvu.edu/etd

\section{Recommended Citation}

Chilakamarri, Raghu, "Actin nanokinematics under the influence of DC electric fields" (2005). Graduate Theses, Dissertations, and Problem Reports. 1658.

https://researchrepository.wvu.edu/etd/1658

This Thesis is protected by copyright and/or related rights. It has been brought to you by the The Research Repository @ WVU with permission from the rights-holder(s). You are free to use this Thesis in any way that is permitted by the copyright and related rights legislation that applies to your use. For other uses you must obtain permission from the rights-holder(s) directly, unless additional rights are indicated by a Creative Commons license in the record and/ or on the work itself. This Thesis has been accepted for inclusion in WVU Graduate Theses, Dissertations, and Problem Reports collection by an authorized administrator of The Research Repository @ WVU. For more information, please contact researchrepository@mail.wvu.edu. 


\title{
Actin Nanokinematics under the influence of DC Electric fields
}

\author{
by \\ Raghu Chilakamarri \\ Thesis submitted to the \\ College of Engineering and Mineral Resources \\ at West Virginia University \\ in partial fulfillment of the requirements \\ for the degree of \\ Master of Science \\ in \\ Electrical Engineering \\ Professor Lawrence A. Hornak, Ph.D, Chair \\ Professor Parviz Famouri, Ph.D. \\ Professor Aaron Timperman, Ph.D (Chemistry) \\ Professor Dimitris Korakakis, Ph.D
}

Lane Department of Computer Science and Electrical Engineering

$$
\text { Morgantown, West Virginia }
$$

2005

Keywords: Actin, Actin with DC electric fields, Myosin 


\author{
ABSTRACT \\ Actin Nanokinematics under the influence of DC Electric fields \\ by

\section{Raghu Chilakamarri \\ Master of Science in Electrical Engineering} \\ West Virginia University

\section{Professor Lawrence A. Hornak, Ph.D., Chair}

Molecular shuttles are nanometer-sized machines capable of transporting single molecules over small distances under user control. Actin-myosin system is a motor protein system, which naturally evolved within the cell for the nanoscale transport. The Acto-Myosin system is fuelled by ATP, converting chemical energy into linear motion. The gliding geometry movement of actin wherein the tails of the motors are adsorbed to the surface and the heads of the motors move the actin filaments across the surface can be exploited to achieve the directional control. The motion of actin in a specific direction and the surface of travel can be modified either through lithographic methods or imprinting techniques. Additionally it has been shown that the direction control can be achieved by the use of electric fields. The electric field exerts a Lorentz force on the negatively charged actin filaments which increases or decreases the filament speed depending on the direction of motion with respect to the electric field. The displacements of filaments following Brownian trajectories can be controlled using electric fields. The aim of this thesis is to estimate and quantize the motion of Actin filaments under electric force fields. The required electrical fields are simulated and the mobility of actin under the electric force field is estimated quantitatively. Experimental exploration of this motion was targeted. 


\section{Acknowledgements}

I express my sincere appreciation to my thesis advisor, Dr. Lawrence Hornak, for his excellent advice, encouragement and moral support throughout my master's program. I would also like to extend my appreciation to the committee members Dr. Parviz Famouri Dr. Dimitris Korakakis, and Dr. Aaron Timperman for their valuable guidance and criticism. I also wish to acknowledge Dr Hideyo Takatsuki who devoted immense time and effort in this research.

I sincerely acknowledge Dr. Kolin Brown for his co-operation and necessary feedback throughout my research.

I express my sincere thanks for the timely help extended by all my friends, without which my thesis completion would not have been this easy. 


\section{Dedication}

It is with tremendous love and affection that I dedicate this work to my parents, and my sister without their endless support and encouragement that they have given me throughout all phases of my education, I would never have come this far. 


\section{CONTENTS}

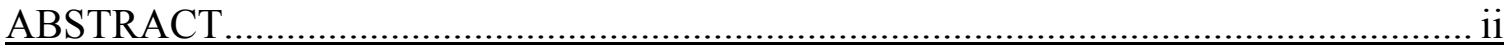

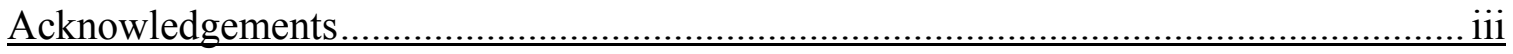

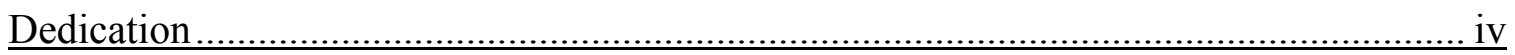

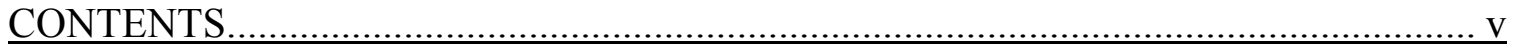

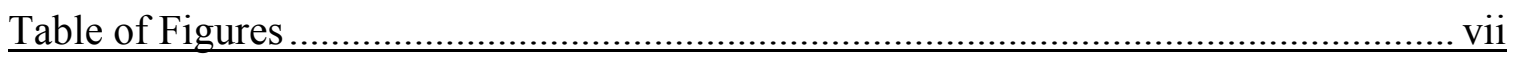

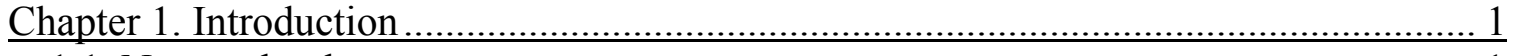

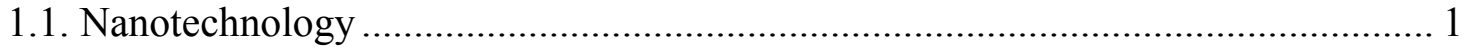

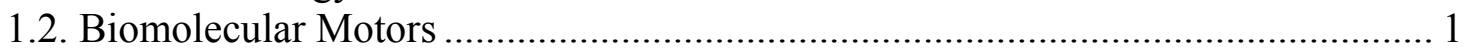

1.3. Proposed work and current motivation of the project......................................... 4

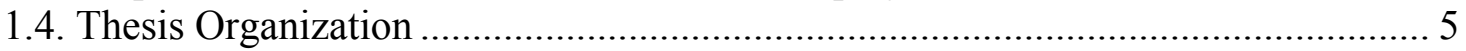

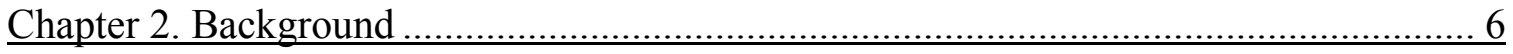

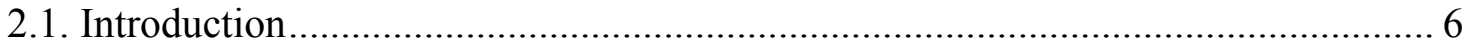

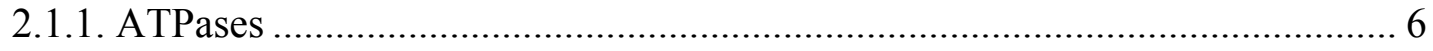

2.1.2. Hydrolysis .......................................................................................... 7

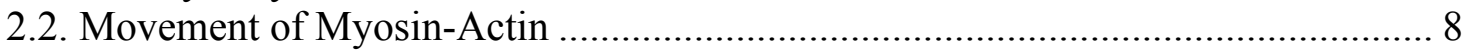

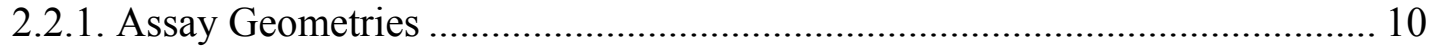

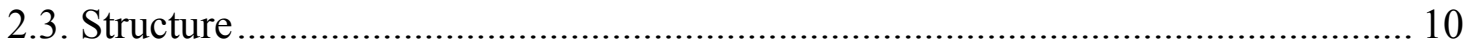

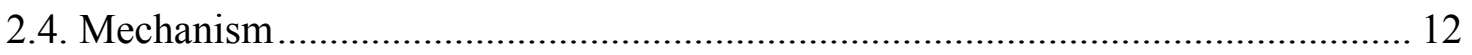

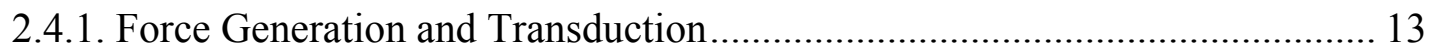

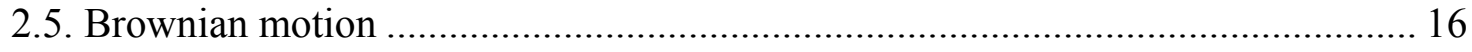

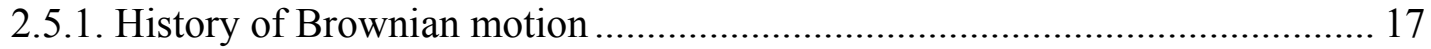

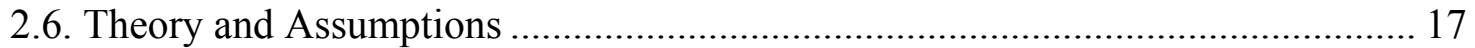

2.6.1. Brownian motion in Velocity Space: Fokker Planck Equation ..................... 19

2.7. Graphical description of Brownian motion in 2 and 3-Dimensions .................... 20

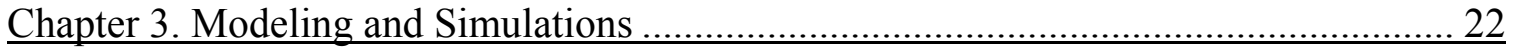

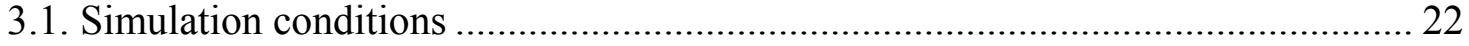

3.2. 2-D Simulations of Chrome Macro electrode structures ................................... 23

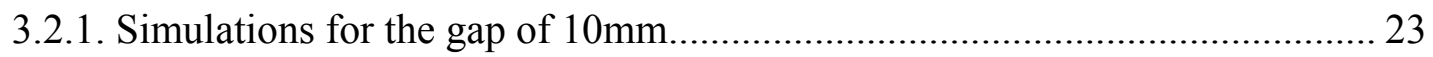

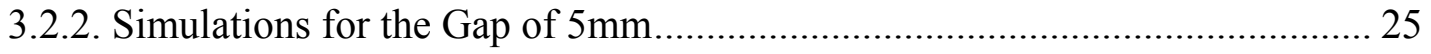

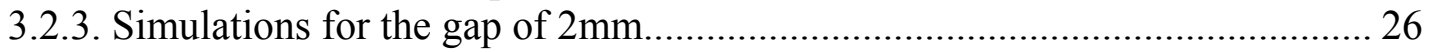

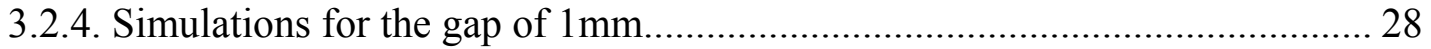

3.3. 3-D Simulations of Chrome Macro electrode structures. .................................... 29

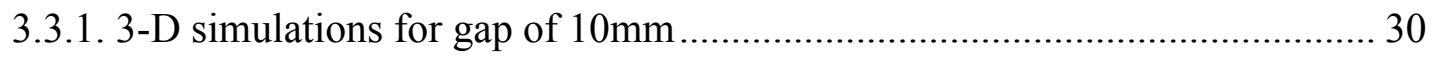

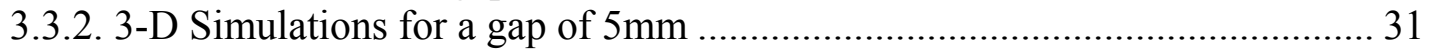

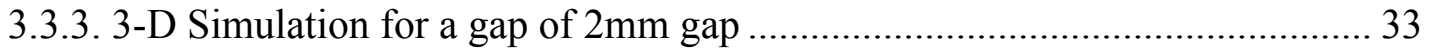

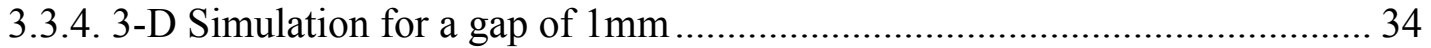

3.4. 2-D Simulations of Gold-Chrome Microelectrode Structures ............................ 36

3.4.1. Simulations of microelectrodes with gap 10 microns ................................. 36

3.4.2. Simulations of microelectrode with gap of 20 microns ............................... 39

3.4.3. Simulation of microelectrodes with gap of 40 microns ................................ 41 
Chapter 4. Design and Fabrication ................................................................................... 43

4.1. Gold Chrome Microelectrode Mask Design ............................................................ 43

4.2. Chrome Macro Electrode Mask design................................................................. 45

4.3. Fabrication of Micro and Macro Electrode structures .......................................... 47

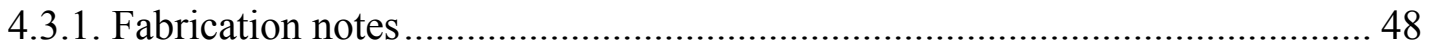

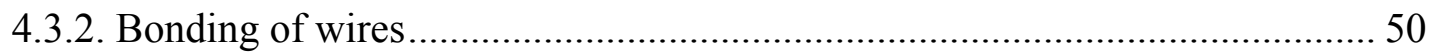

4.3.3 Fabrication of Macro electrode structures …………....................................... 51

4.3.4. Bonding of wires to the Chrome slides....................................................... 51

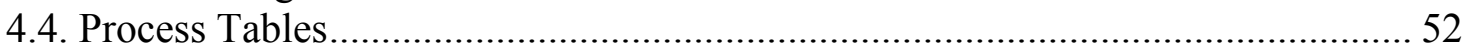

Chapter 5. Experimental Setup ………………………………………………………. 54

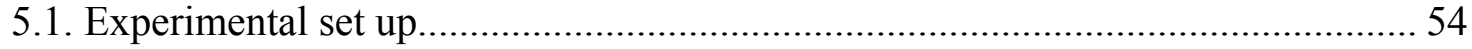

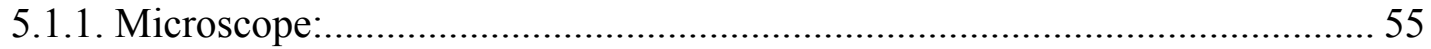

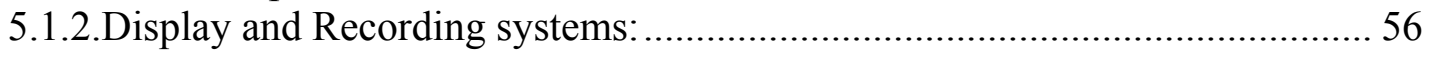

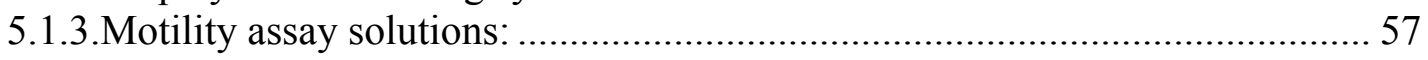

5.1.4. Nitrocellulose (Collodion)-coated coverslip .................................................. 57

5.2. Buffer Solutions and their importance in the Mobility Assays................................ 58

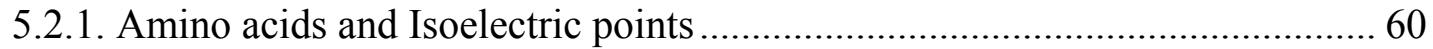

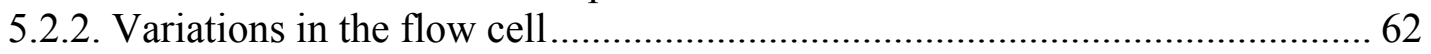

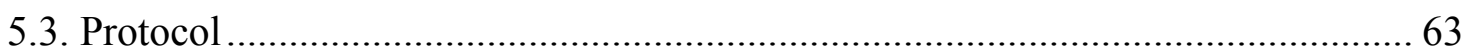

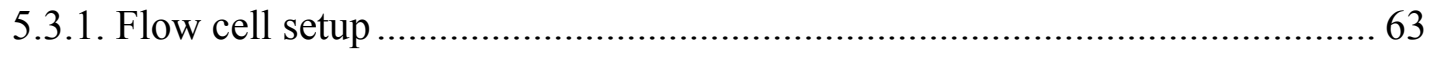

5.3.2. Perfusion of respective solutions to flow cell between glass coverslip and

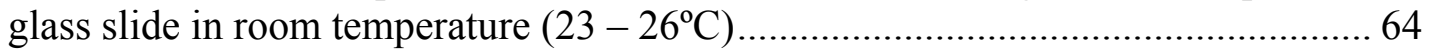

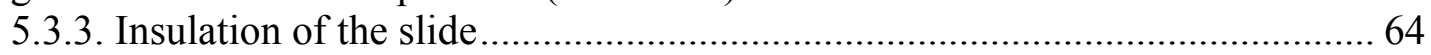

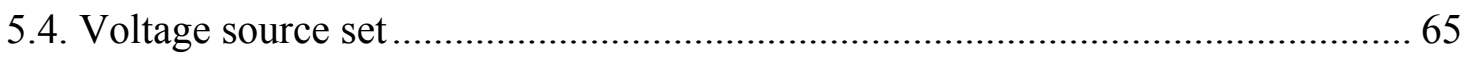

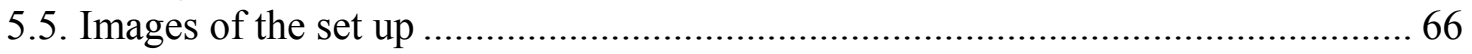

Chapter 6. Experimental Results and Discussion ............................................................ 67

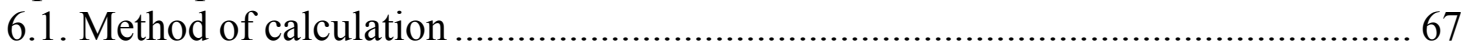

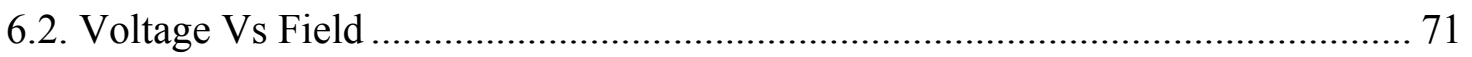

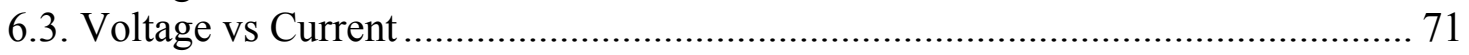

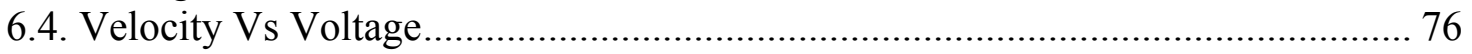

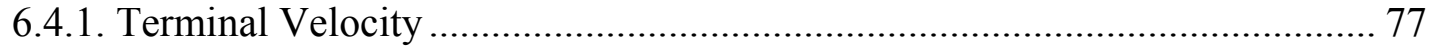

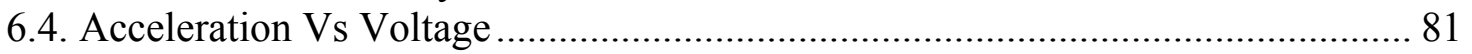

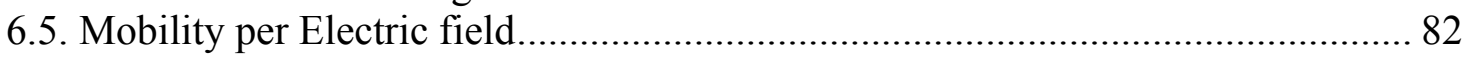

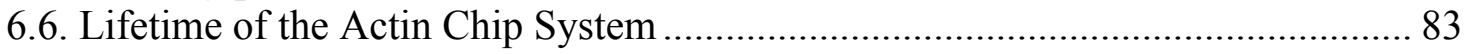

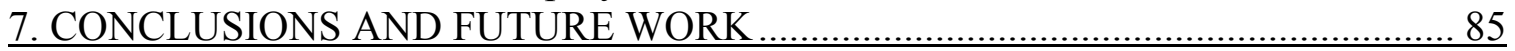

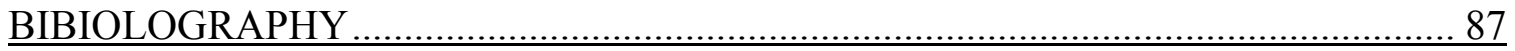

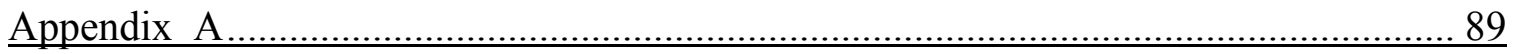

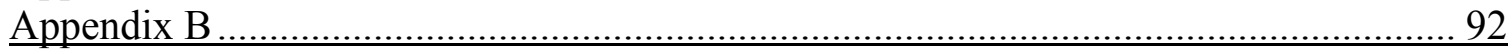

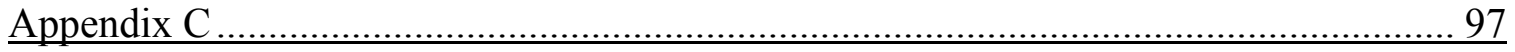




\section{$\underline{\text { Table of Figures }}$}

Figure 1 Graphical representation of Myosin II [9] ................................................. 8

Figure 2 Graphical representation of actin gliding over myosin; adapted from[9] .......... 10

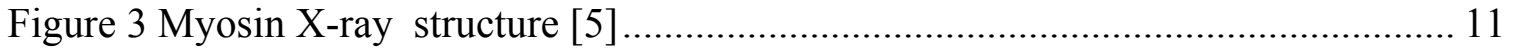

Figure 4 Below: Myosin Structure[5] ........................................................................... 11

Figure 5 Change in the twist angle $\varphi$ due to hydrolysis, represented by the angle, adapted

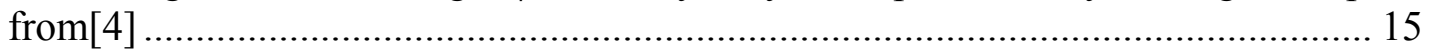

Figure 6 Change in the angle $\theta$ causing the motion of the myosin on actin; adapted

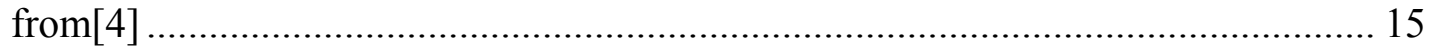

Figure 7 Simulation of Brownian motion in 2-D space................................................ 20

Figure 8 Simulation of Brownian motion in 3-d space............................................ 21

Figure 9 10mm gap Applied Voltage = 3V ........................................................ 23

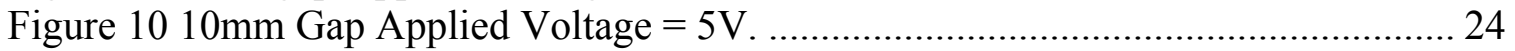

Figure 11 10mm Gap Applied Voltage $=7 \mathrm{~V}$........................................................ 24

Figure 12 5mm Gap Applied Voltage = 3V ……………….................................... 25

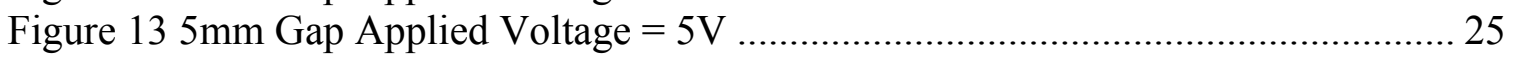

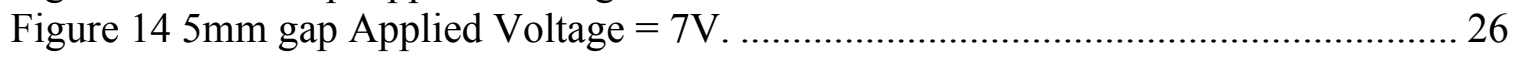

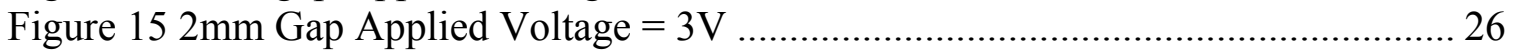

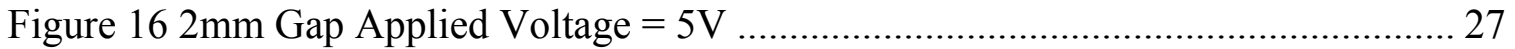

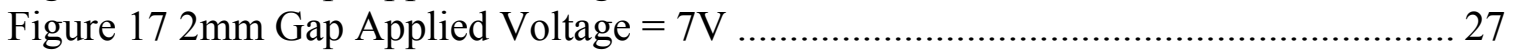

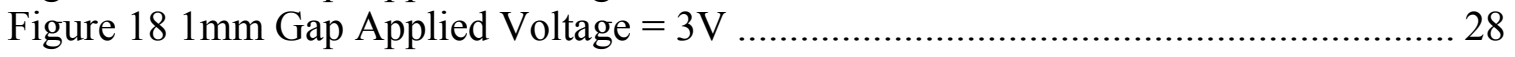

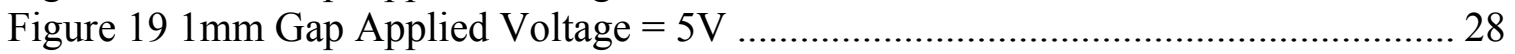

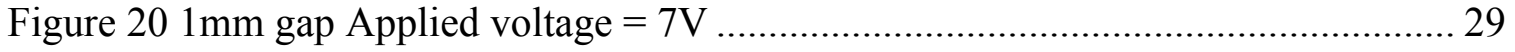

Figure 21 3-d 10mm Gap AppliedVoltage = 3V ................................................... 30

Figure 22 3-d 10mm Gap Applied Voltage $=5 \mathrm{~V}$........................................................ 30

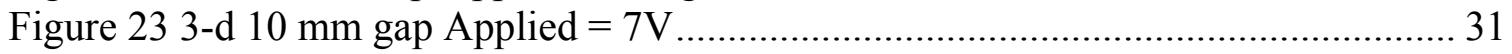

Figure 24 3-d 5mm Gap AppliedVoltage = 3V ………........................................... 31

Figure 25 3-d 5mm gap Applied Voltage $=5 \mathrm{~V}$......................................................... 32

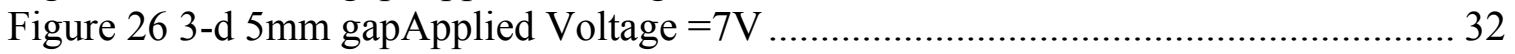

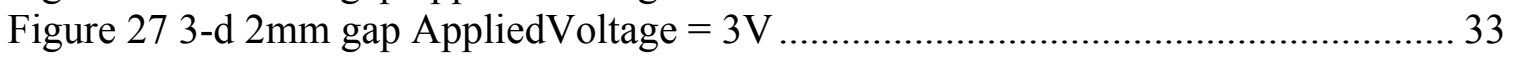

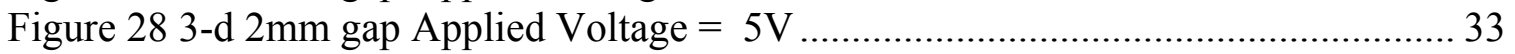

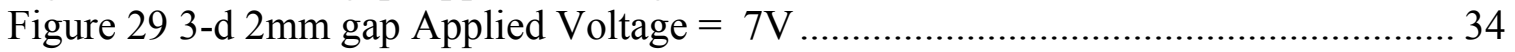

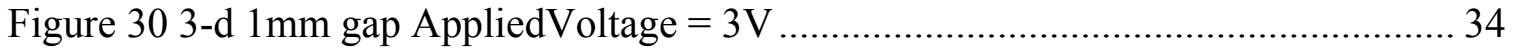

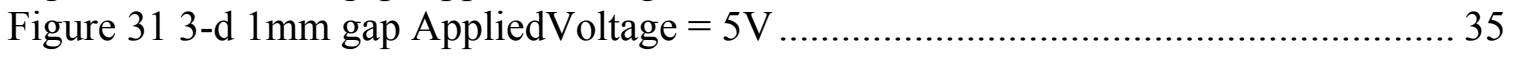

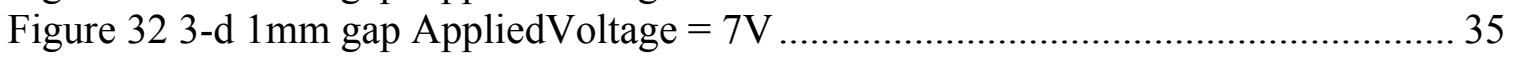

Figure 33 Index of the simulated microelectrode structures............................................. 36

Figure $3410 \mu \mathrm{m}$ gap Applied Voltage $=0.5 \mathrm{~V}$.......................................................... 37

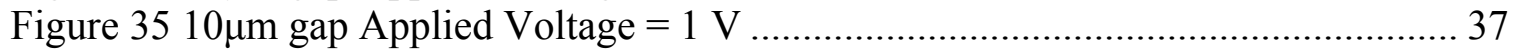

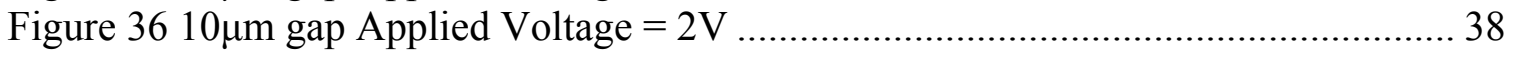

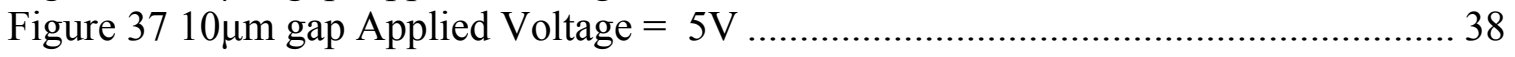

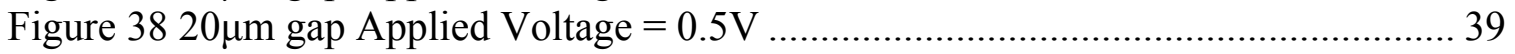

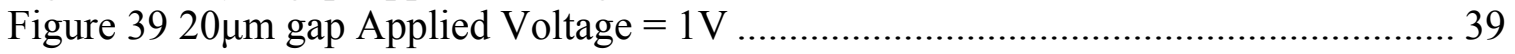

Figure $4020 \mu \mathrm{m}$ gap Applied Voltage $=2 \mathrm{~V}$........................................................ 40

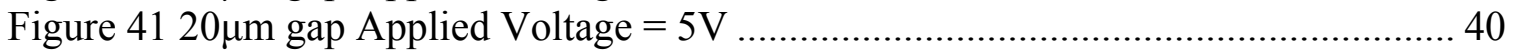

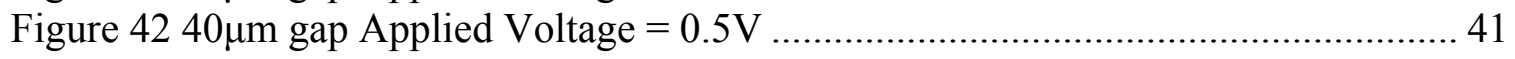




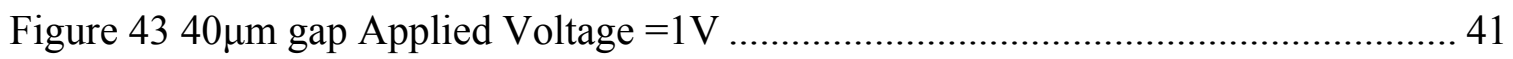

Figure $4440 \mu \mathrm{m}$ gap Applied Voltage $=2 \mathrm{~V}$............................................................. 42

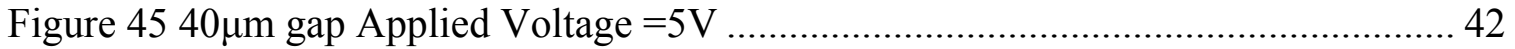

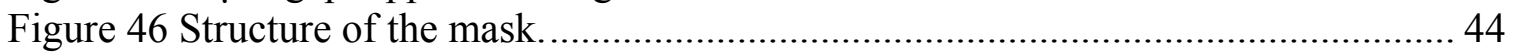

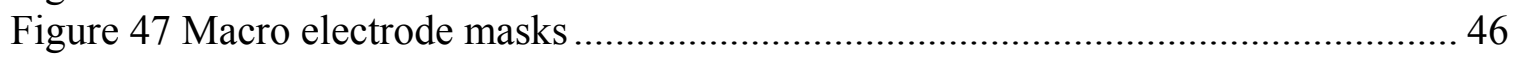

Figure 48 Graphical representation of the process flow of the microelectrode fabrication

Figure 49 Electrode structures on glass slide after fabrication ....................................... 48

Figure 50 Electrode structure after $\mathrm{SiO}_{2}$ deposition and after bonding the wires ............ 50

Figure 52 Macro electrode slide $1 \mathrm{~mm}$ gap with the wires bonded with epoxy ................ 51

Figure 53Graphical representation depicting the setup for a standard motility assay ...... 54

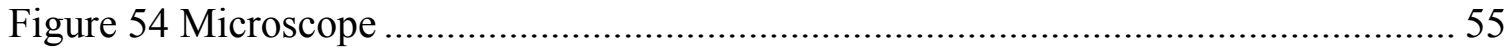

Figure 55 Flat screen Monitor used for analyzing the videos.......................................... 56

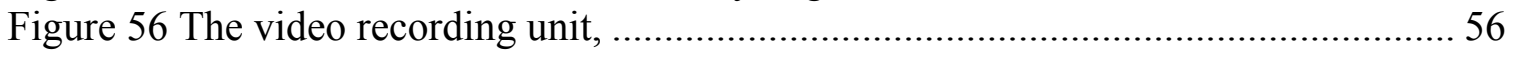

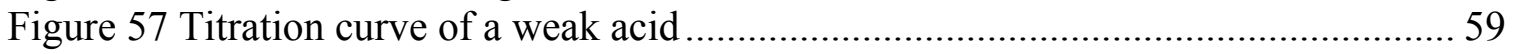

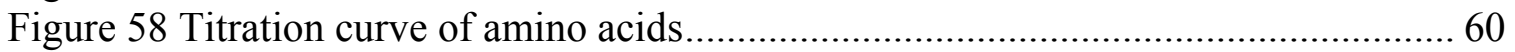

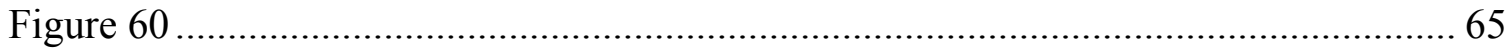

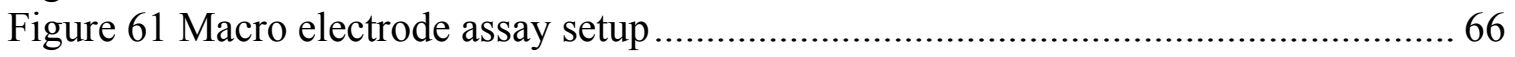

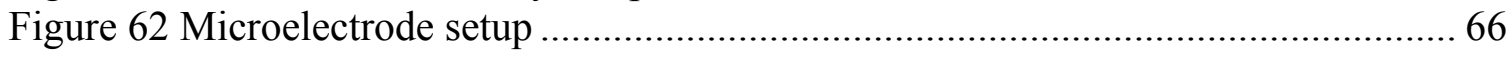

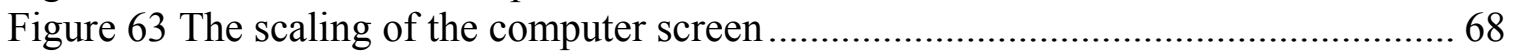

Figure 64 Method of markup of the distance traveled.................................................... 69

Figure 65 Plot of Electric Field vs Voltage ............................................................. 71

Figure 66 Current vs Voltage in the flow cell with pH7.2, Normal buffer conc, and with

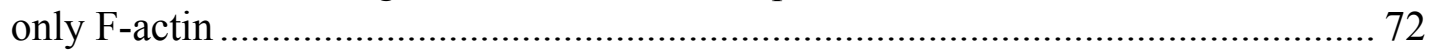

Figure 67 Current vs Voltage in the flow cell with pH7.2, Normal buffer conc, and with BSA+ F-actin+ Collodion ........................................................................... 73

Figure 68 Current vs Voltage in the flow cell with varying pH, Normal buffer conc, and

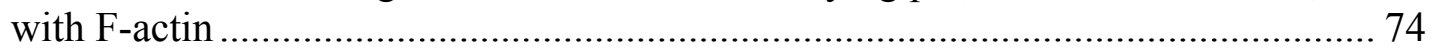

Figure 69 Current vs Voltage in the flow cell with $\mathrm{pH} 7.2$, and varying buffer conc, and

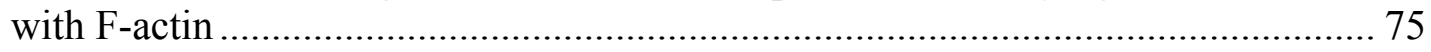

Figure 70 Plot of Velocity Vs Voltage under normal concentration of buffer, $\mathrm{pH} 7.2$ with

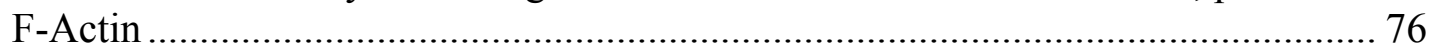

Figure 71 Error bars for the velocity Vs Voltage graph ................................................ 78

Figure 72 Variation of velocity with $\mathrm{pH}$ for $5 \mathrm{~mm}$ gap, with F-actin only...................... 79

Figure 73 Variation of velocity with buffer concentration for $5 \mathrm{~mm}$ gap, $\mathrm{pH} 7.2 \ldots \ldots \ldots \ldots . .80$

Figure 74 Variation of acceleration in different gaps between electrodes, pH 7.2, F-actin

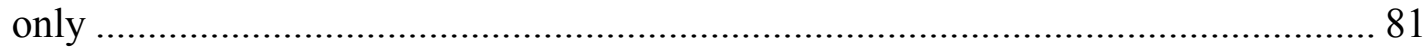

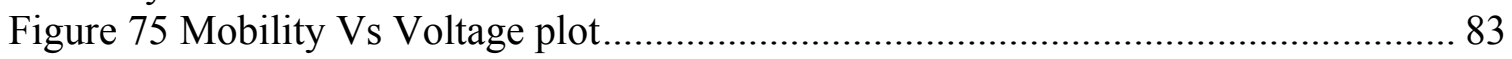




\section{Chapter 1. Introduction}

\subsection{Nanotechnology}

The need for smaller and faster devices has been the trend of the techno world since the inception of Moore's law. Smaller devices have always been advantageous for they occupy less space and easy to handle, but they also come with some trade offs like high cost, flimsiness of the device and also increased complexity of building. In the contemporary world ability to hold the tools and manipulate them as required governs the constructions. But in the nano world the problem of big fingers arises. Techniques like the AFM do offer the possibility of manipulating atoms, but they are time consuming and expensive affairs. The possibility of manipulating materials at the atomic level would just have remained a dream if not for the discovery of motor proteins which can be used for nanoscale transport. The possibilities can be endless, to state a few, drug delivery systems that can be more accurate and more miniaturized and user friendly. Self assembly of materials, wherein the composition of the material can be altered at the atom level; this can give rise to desired materials with the desired properties.

\subsection{Biomolecular Motors}

Nanotechnology research in the area of bio nano systems has been an area of intense interest. Nano systems involve the application of biological materials and processes on the nano and micro scale area. Bio nano science is an amalgam of a variety of disciplines of medicine, biology, engineering and physics research. The Bio nano systems currently under focus and development around the world include systems for drug delivery and 
separation, DNA conductors, biological warfare pathogen sensing, and parts delivery for nanoscale manufacturing process. Some of these applications depend on nanoscale transportation systems to facilitate movements of nanometer and micrometer scale elements. [1]

In order to ultimately design artificial systems that can mimic biological transport process, which are interesting and fascinating areas of research, researchers are hoping to gain more rudimentary level of understanding about the governing mechanics of biological transport. [1]

Cells in living organisms synthesize, assemble, and package proteins and protein complexes into membranous vesicles and organelles, for use throughout the cell. [2]. A variety of molecular motors are used to move this cargo throughout the cell. This process is carried out by motor systems built of proteinaceous segments of actin, myosin and tublin. This motor system navigates through an intercellular interstate system. Predominantly the three proteins - Myosin, Kinesin and Dynesin harness the power of Adenosine Triphosphate (ATP) hydrolysis to transport loads over vast intercellular distances. The two protein based linear motor systems under research for use in nanoscale transport systems are the Actin-Myosin system and microtubule-kinesin system. [2] The difference between the kinesin and myosin family is that they have different mechanisms of movement. Kinesins bind microtubules, while myosin attach to actin filaments. The protein system under analysis in this thesis is the Myosin-Actin system. This system was preferred as the breadth of knowledge concerning the proteins is vast. (Myosin was first 
isolated in 1864) Also the assay under consideration is termed a gliding assay. More details about this can be found in Chapter II of this thesis.

Actuation rate control, directional control and cargo attachment to filament/motor molecules are the major requirements for harnessing the motion of bio molecular motors for nanosystems. As these bio molecular systems evolve and mature, it is critical that an interface be established between their nanoscale motion and the chip-level microelectronic environment that will enable reconfigurable control of their nanoscale motion via electronic signaling. To guide the motion of proteins in a specific direction the surface of travel can be modified either through lithographic methods or imprinting techniques.[10],[11]. Additionally it was also determined experimentally in [3] that the directional control can be achieved through electric fields.

But before the study of motility assays are undertaken, it is important to develop an understanding of the response of actin filaments to the electric fields. The actin filaments are the cargo holders in the nanoscale transport system and an insight into the field response would provide more information in the characterization of the motility assay. The mobility assays under different ambiences and different voltages facilitate the characterization. The actin filaments are inherently negatively polarized filaments which provide an opportunity to utilize electric fields to control their mobility. The electric field exerts lorentz force on the actin filaments, which align the filaments and guide them in a specific direction. The macroscopic electro-motility characteristics have already been established [3]. This thesis will to explore more into the control of directionality of the 
actin protein system in an assay due to the forces caused by electric fields in the microscopic level. Mobility response of the actin with respect to applied fields was targeted with various applied voltages and with varying concentration of the buffer solution, in which the protein system survives. Macroelectrode and microelectrode flow cells were fabricated for this purpose. The voltage and electric field patterns of these electrode structures were simulated with the help of a software tool and compared with the theoretical calculations and experimental results.

\subsection{Proposed work and current motivation of the project}

This research studies the mobility of actin protein system when exposed to electric fields, in a conducive protein survival environment, with respect to the voltage applied. The project also includes analysis, modeling, and design of electrode slides. The components of the project are

a. Design of mask for the electrode structures to be fabricated on microscopic slides.

b. Validation of the design geometry using an electric field simulation tool (Ansoft MAXWELL) with simulations for different geometries of electrode structures and for different applied DC voltages.

c. Based on the simulations results, finalizing the specifications of the electrode structure for fabrication of the microelectrode slides on microscopic slides.

d. Construction of the experimental setup for the performing the mobility assays.

e. Performing mobility assays with varied applied voltages and with various concentrations of the buffer solution. 


\subsection{Thesis Organization}

This thesis is organized as follows:

Chapter II starts with an overview of the Myosin-Actin protein system and their transport geometry; their motility characteristics, and concludes with the description of the aspect of the motion.

$>$ Chapter III describes deals with the electrostatic simulations performed to arrive at the design parameters for chip fabrication.

Chapter IV describes the design of the mask for the electrode structures, the methods employed to fabricate the chips, the way to connect the chips to voltage sources.

$>$ Chapter V describes the experimental setup of the project.

Chapter VI analyses the results obtained from the setup in chapter IV and discusses them in detail

Chapter VII describes the conclusions drawn using the results and also discusses anticipated work in the future. 


\section{Chapter 2. Background}

\subsection{Introduction}

Molecular shuttles or nanoscale motors are motor proteins capable of transporting molecule(s) over small distances under user control. The movement of these proteins is through a cellular system which is built of proteinaceous segments of actin and tubulin. The movement sometimes is intercellular too. The three major protein systems that travel this way are Myosin, Kinesin and Dynesin. Adenosine Triphosphate (ATP) serves as the fuel for their movement, and transportation of loads larger than themselves over relatively larger distances. The mechanism of ATP hydrolysis will be explained in more detail later in the chapter. The research aimed at nanoscale transport systems on the basis of motor proteins has in general chosen the proteins from the well-known Kinesin and Myosin family. These motor proteins use ATP and convert the chemical energy to linear motion with more than $50 \%$ efficiency. However for in vitro applications nanoscale transport is limited by the need for a defined environment resembling the conditions in cells. The proteins used in our experiments aimed at Nanoscale directional control, belong to the family of Heavy Meromyosin (HMM). The advantages and reasons for using HMM are (i) there is a good amount of information available on Myosin (Myosin II was first isolated in 1864) (ii) there is a possibility to produce significant quantities of proteins by recombinant methods used for DNA. [2]

\subsubsection{ATPases}

The force required for the functioning of motor proteins like Myosin and Kinesin is generated hydrolyzing ATP, thus they are ATPases. ATPases are a class of enzymes that 
decompose Adenosine Triphosphate (ATP) into Adenosine Diphosphate (ADP) and a free phosphate ion. This reaction releases energy, which the enzyme (in most cases) utilizes to drive other chemical reactions that depend on the released energy. This process is widely used in all known forms of life.

\subsubsection{Hydrolysis}

The chemical reaction in which the action of water or its ions breaks down a substance into smaller molecules is termed as Hydrolysis. Hydrolysis occurs in certain inorganic salts in solution, in nearly all non-metallic chlorides, in esters, and in other organic substances. This mechanism is also used by the human body for the breakdown of food during the conversion of starch to glucose. The movement of the actin-myosin system is made possible by the hydrolysis of Adenosine Triphosphate (ATP) into Adenosine Diphosphate $(\mathrm{ADP})+\mathrm{Pi}$, where $\mathrm{Pi}$ is inorganic phosphate. ATP acts as fuel to the system. The overall energy equation of a general hydrolysis of ATP is given as follows.[8]

$$
\begin{aligned}
& A T P+H_{2} \mathrm{O} \Leftrightarrow A D P+\text { inorganicphosphate }(P i) \\
& \Delta G^{t}=\Delta G^{o t}+R T \ln \frac{[A D P][P i]}{[A T P]} \\
& \Delta G^{o t}=-7.3 \text { kcal.mol }^{-1}
\end{aligned}
$$

where $\Delta G^{o t}$ is the free energy of hydrolysis of ATP.

The hydrolysis reaction cycle causes a power stroke against a load. The maximum limit for the work performed during the power stroke is limited by the free energy of the 
hydrolysis process. The efficiency or the energy utilization of the enzymes of kinesin, myosin is high. The efficiency exceeds $50 \%$ in cases of kinesin.

\subsection{Movement of Myosin-Actin}

A large number of Kinesins and Myosins with different structures and specialized functions were discovered. The distinctive properties of proteins are based on the structure. The difference in Kinesin family and Myosin family is that they have different methods of movement. The protein Kinesin binds to microtubules, while the protein Myosin attaches to actin filaments. Actin filaments are polymer filaments assembled from smaller sub protein units of tubulin and actin monomers. The length of an actin monomer is usually $5.5 \mathrm{~nm}$. They polymerize forming double stranded ropes twisted every $72 \mathrm{~nm}$. There is a dynamic and regulated equilibrium in the cell between polymerization and de-polymerization causing a constant recycling of tubulin and actin, forming new polymer strands [2].

The movement of motor protein Myosin II-Actin system is termed as non-processive or running. As discussed earlier the hydrolysis of ATP causes the movement of the MyosinActin system. This hydrolysis causes a small conformational change in the structure of the myosin. The structure of myosin is graphically depicted in the picture below.

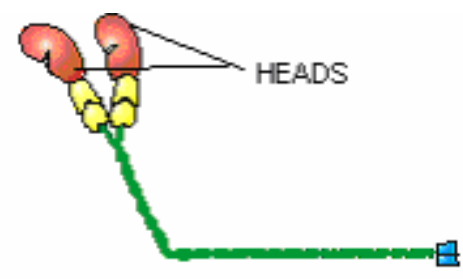

Figure 1 Graphical representation of Myosin II [9] 
The structural or conformational change caused by hydrolysis process is amplified by the lever arm which connects the head binding to the actin to the tail region. The hydrolysis cycle effects a change in the attachments of the head to the actin filaments accompanied by a conformational change. This leads to an uniform periodic attachment and detachment of the motor causing displacement in a defined direction. Different motors have different characteristics depending on the varying lengths of the lever arms, the attachment and detachment rates, and the formation of proteins dimers from monomers. The Myosin II motors act together and they are not usually in a permanently attached state. The Heavy Meromyosin or HMM is a subunit of Myosin II. As mentioned earlier the actin filaments consists of two strands, polymerized from actin subunits twisted around each other. The myosin moves over or causes the actin to move over them (in case of gliding geometry). These proteins have to be kept in a solution of Cytosol. Cytosol is the fluid portion of cytoplasm without organelles and membranes. Care has to be taken so that the $\mathrm{pH}$, salt content and temperature stay within a certain range and denaturing substances have to be avoided. The $\mathrm{pH}$ typically for actin has to stay between $\mathrm{pH} 5.4$ and $\mathrm{pH} 8.0$. The denaturing of the proteins is also caused due to the contact between proteins and bare synthetic surfaces, so surface properties have to be controlled. A membrane of colloidion usually acts as an interface between the proteins and synthetic surfaces. The proteins are kept frozen otherwise they degrade within hours to days due to oxidation and the action of proteases present in trace amounts. The concentrations of ATP and ADP + Pi have an influence on the activity of enzyme [2]. 


\subsubsection{Assay Geometries}

Motility assays of proteins employ two different geometries called the bead assay and the gliding assay. The movement of the motors on the microtubules or filaments fixed to the surface is characterized as the bead assay. Beads are bound to one or more motors for visualization of the movement. In the gliding assay the heads of motors move the actin filaments or microtubules across the surface while the tails of the motors are adsorbed to the surface. The assay targeted in our study is the gliding assay. Adsorption of microtubules of actin filaments into complex patterns is reportedly difficult to achieve and in the bead geometry the uninterrupted movement of a single motor is limited to the length of a fiber, for this reason the gliding geometry is preferred [2]. The illustration of the gliding geometry is as follows.

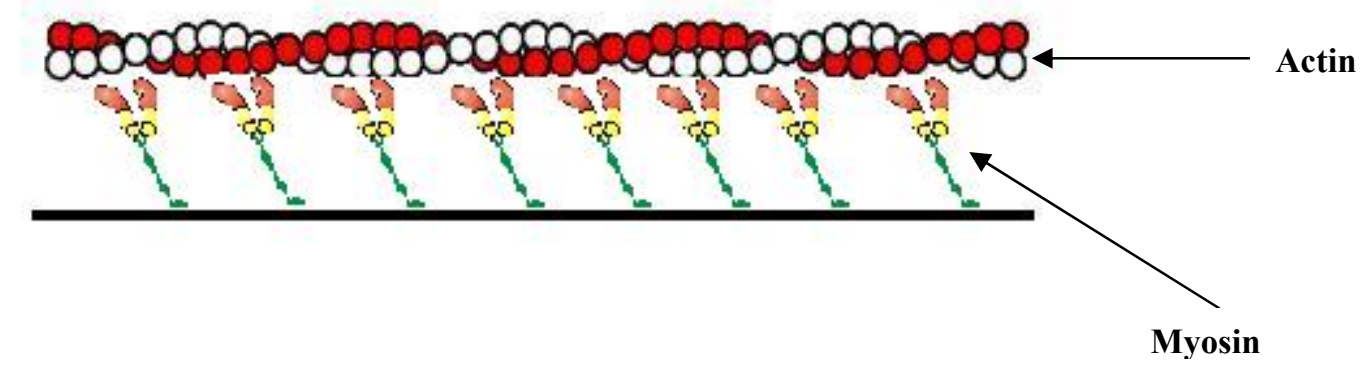

Figure 2 Graphical representation of actin gliding over myosin; adapted from[9]

\subsection{Structure}

Myosin is an asymmetric protein composed of two globular heads attached to a long tail which forms the thick filament; Actin is a double-helical protein making up the thin filament. The force generation in muscles involves interactions between actin, and myosin. The myosin molecular weight is approximately $500,000 \mathrm{Da}\left(1\right.$ Dalton $=1 / 12^{\text {th }}$ mass of carbon atom of Carbon-12) and it consists of six polypeptide chains: two heavy 
chains with a mass of $400,000 \mathrm{Da}$ and two sets of light chains, each weighing about 20,000Da. The two globular heads consists of about 850 amino acid residues each, each head contains a heavy chain associated with two light chains. A $150 \mathrm{~nm}$ long rod is formed by the intertwining of bulk of one head, which is formed by the heavy chain [4].

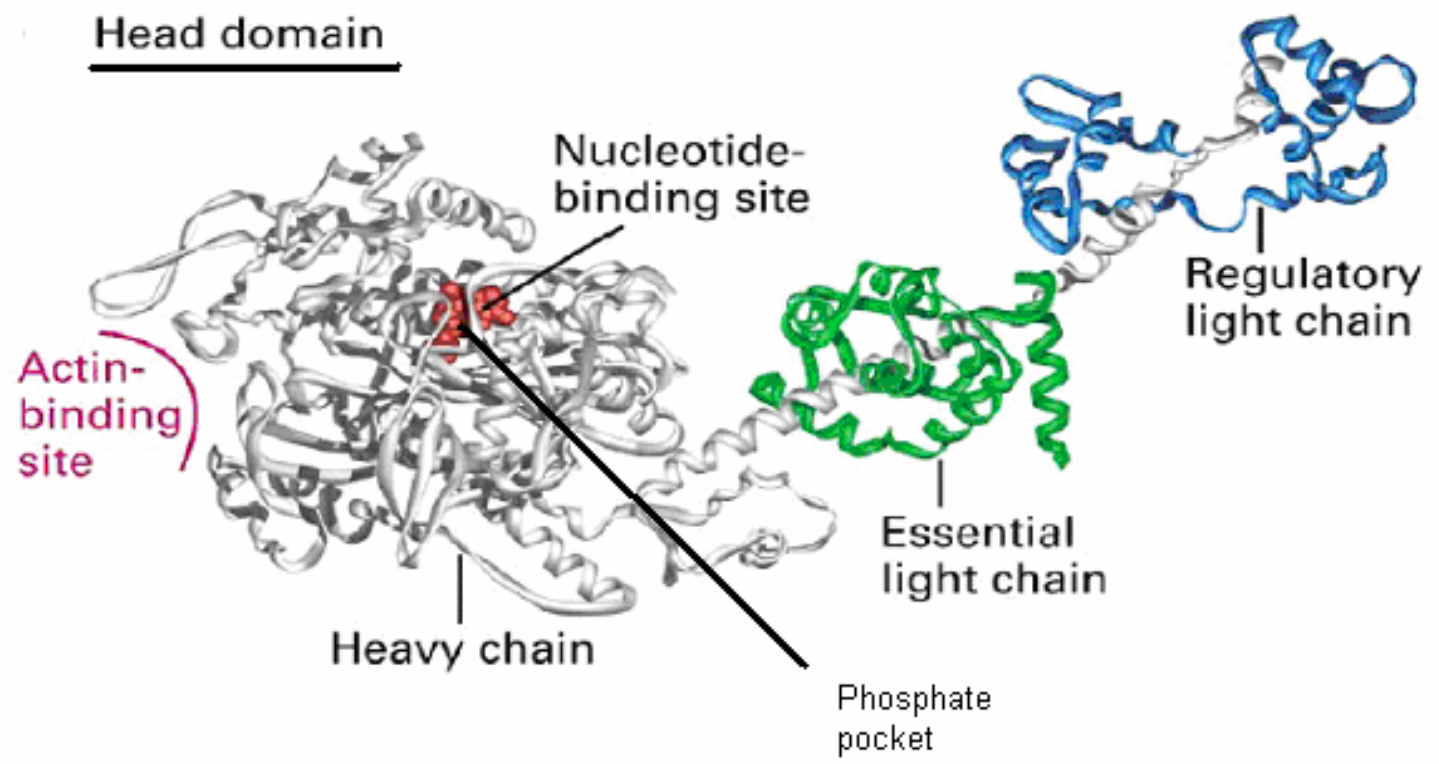

Figure 3 Myosin X-ray structure [5]

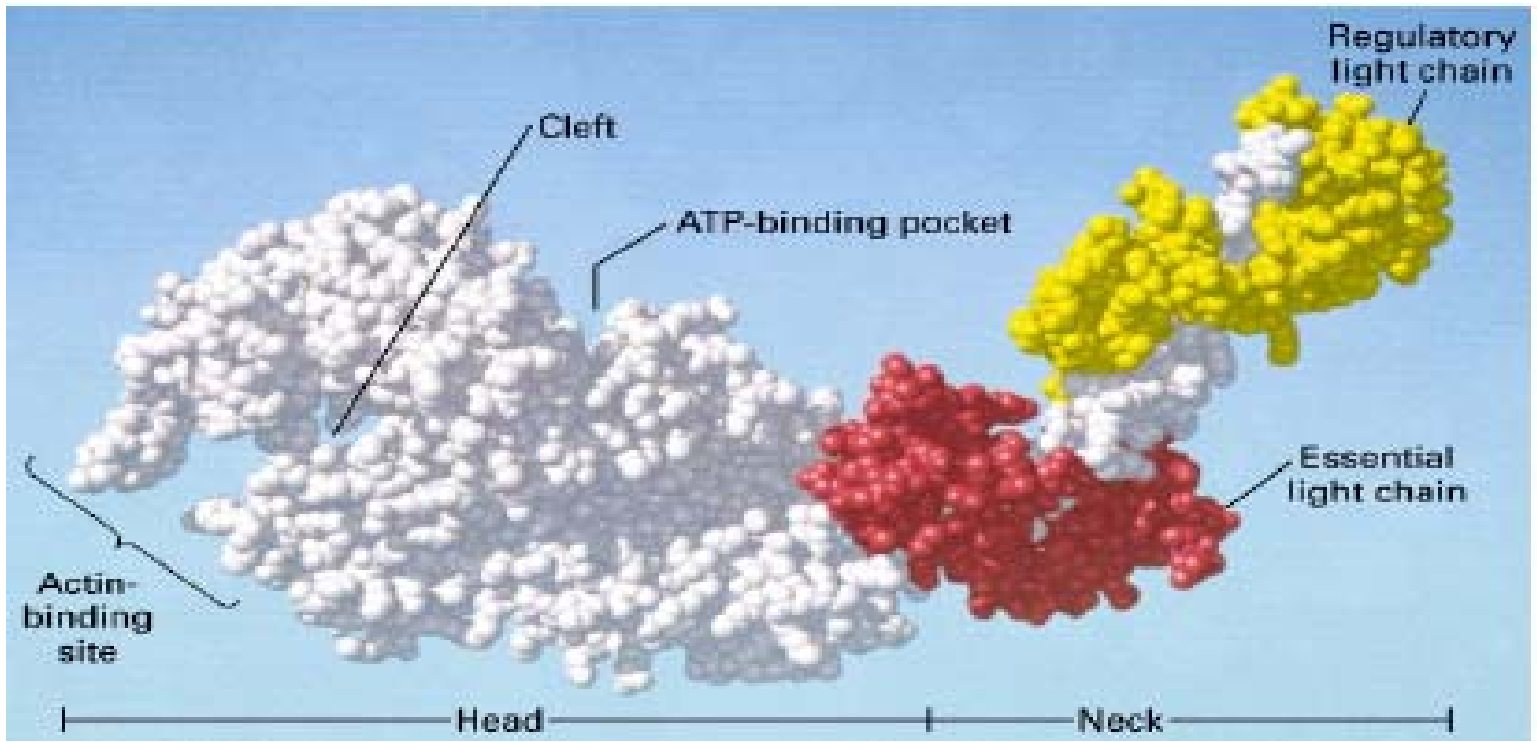

Figure 4 Myosin Structure[5] 
The myosin head contains of an ATP binding site, actin and two light chain binding sites, and the myosin rod consists of a coiled-coil of two $\alpha$ helices. Several deep gaps and pockets can be observed from the skeletal structure of the myosin head. The $\alpha$ helix, which is the as regulatory domain or the lever arm, is composed of more than 60 amino acid residues[4]. This extends from the thick part of the head, which is the catalytic domain containing the nucleotide and actin binding sites to the $\mathrm{COOH}$ terminus of head fragment. The head is composed of three major regions: a $25 \mathrm{kDa} \mathrm{NH}_{2}$ terminal domain which contains the nucleotide binding site, a $20 \mathrm{kDa} \mathrm{COOH}$ terminal domain, and a central $50 \mathrm{kDa}$ segment that is split into an upper and a lower domain by a prominent gap (50K gap) that extends from the base of the nucleotide binding pocket to the actin binding interface [4]. This along with the $20 \mathrm{kDa}$ domain is the actin binding site of the molecule, which lies on the opposite side of the nucleotide binding site. The molecule is made up of a fibrous rod-like tail region whose amino acid sequence is highly repetitive [4]. The 50kDa gap serves as the communication between the actin binding interface and the nucleotide binding site by means of domain movements associated with it. These domain movements are such that binding of actin to myosin closes the 50kDa gap and opens the nucleotide binding site, while binding of nucleotide closes the nucleotide binding site and disrupts the actin binding interface by opening the 50KDa gap. [4]

\subsection{Mechanism}

The explanation of the mechanism begins from the point where the nucleotide binding site of the myosin molecule has an ATP molecule bound to it and the myosin molecule is ready for hydrolysis. The molecular mechanism of ATP hydrolysis causes structural 
changes, and the conformational changes accompanying it were reportedly observed by X-ray structural studies. [4]

The major conformational change between the two stages of hydrolysis occurs in the $\gamma$ phosphate pocket and the Mg ADP leaving group experiences a very similar environment in the two states. Thus, when ATP hydrolysis takes place, the phosphorous to oxygen distance increases on transition from ATP to the ADP + Pi state. The conformational change in the $\gamma$-phosphate pocket causes the movement of the lower 50kDa domain relative to the upper $50 \mathrm{kDa}$ domain, resulting in partial closure of the $50 \mathrm{~K}$ gap. This change is propagated to the $\mathrm{COOH}$ terminal region of myosin causing a major rearrangement of the polypeptide chain. This causes the top of the regulatory domain of the myosin head to rotate by $\sim 20^{\circ}$. After hydrolysis the conformational changes caused by binding of actin to myosin detaches the MgADP and Pi from the site [4].

\subsubsection{Force Generation and Transduction}

The myosin molecule possesses a unique mechanism to store the energy of ATP hydrolysis within itself as internal energy. The ATP hydrolysis takes place when the myosin is not attached to actin. But for the system to function i.e. for the movement of actin on myosin to take place, myosin has to be attached to actin. So this situation provides the basis of argument that the energy has to be stored internally or else there would be dissipation of energy resulting in no useful work done. An implicit feature of all kinetic schemes till date has been the presence of a time lag between the hydrolysis event and the motion. This feature lends further support to the energy storage principle. 
The energy was proposed to be stored in the coiled coil of the S-2 region of myosin rod. It is stored as an increase in twist of the $\alpha$-helices of the myosin rod. The ATP hydrolysis causes a rotation of the top of the regulatory domain which leads to an increase in the twist in the myosin rod and subsequently the rotation of myosin head. A tilt of the myosin head accompanies the increase in twist of the coiled coil. The region connecting myosin head to the rod region (connecting S-1 to S-2) experiences the tilt. Therefore the ATP hydrolysis has three effects

1. Increase in the twist of $\alpha$ - helices forming myosin rod.

2. Rotation of the myosin head.

3. Tilt of the myosin head.

For a more quantitative approach, let us define the twist angle $\varphi$ as the angle between the axis of the rod and the tangent to one of the $\alpha$-helices of the coiled coil. The angle $\varphi$ decreases when there is an increase in the twist. This can be observed in the figure of prehydrolysis and post hydrolysis stages. The angle $\theta$ is the tilt angle defined as the angle formed by the axis of the actin filament with the lever arm. The energy released due to the enthalpy change upon ATP hydrolysis causes a decrease in $\varphi$ which accounts for the stored energy. The myosin head is brought closer to the actin filament by the decrease in $\theta$ and due to the rotation of myosin head. This facilitates the attachment and binding of myosin to actin. Therefore tilting, twisting and rotation are the critical acts in this molecular mechanism. When this mechanism takes place and when myosin is bound to actin, it leads to a complete closure of the previously partially closed 50K gap and opens up the nucleotide binding site and releases bound ADP + Pi.[4] 


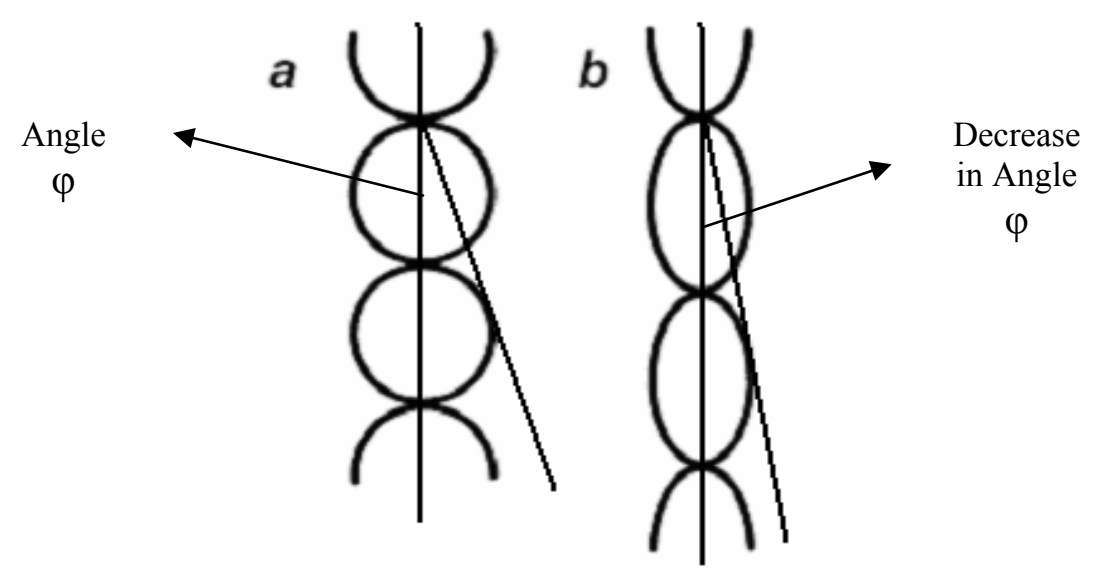

Figure 5 Change in the twist angle $\varphi$ due to hydrolysis, represented by the angle, adapted from[4]
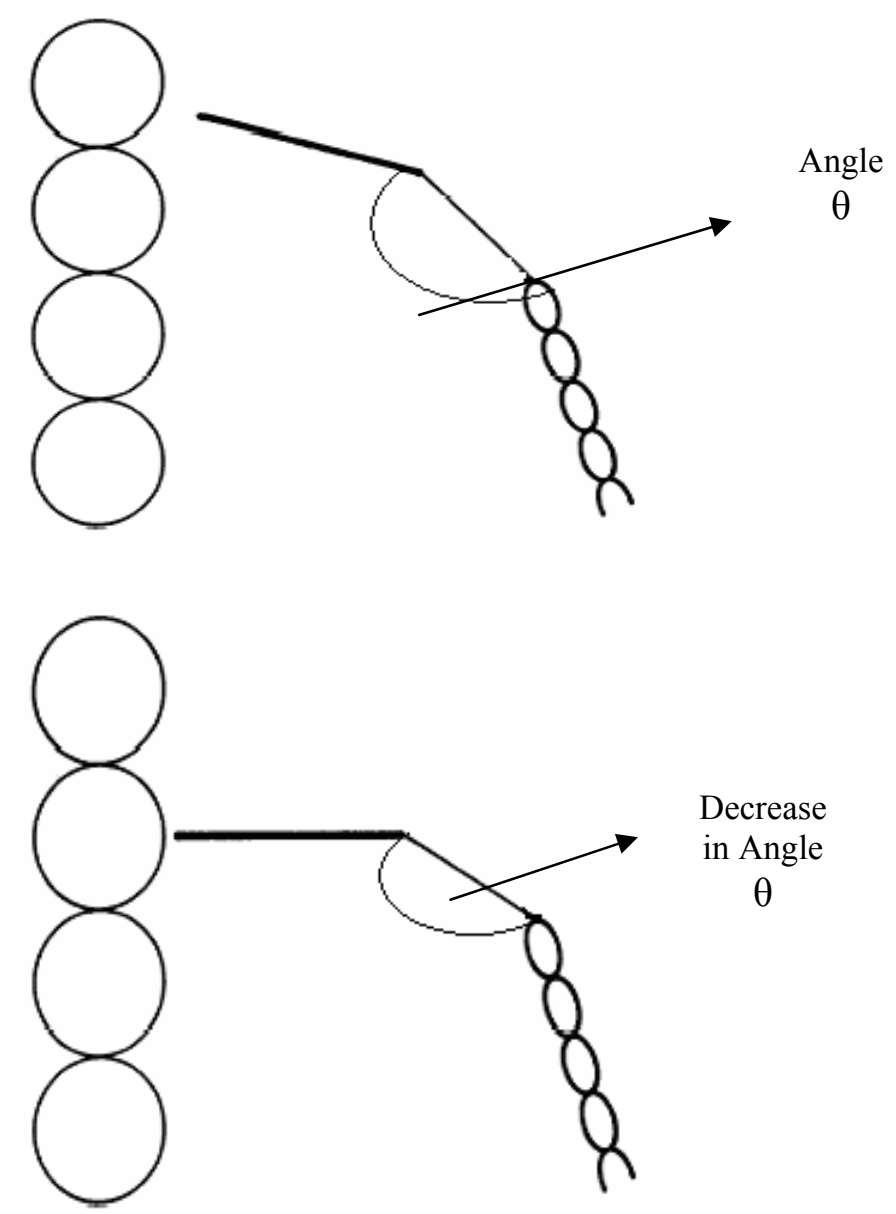

Figure 6 Change in the angle $\theta$ causing the motion of the myosin on actin; adapted from[4]

The increase in $\theta$ is an indicator of the power stroke taking place, i.e. when the S-2 region coiled coil untwists. Stored energy, which is used to move the actin filament, is released 
when there is a decrease in twist. Accompanying the untwisting the angle $\theta$ increases i.e. the myosin head untilts about the contact between S-1 and S-2. The actin filament along with the myosin head is dragged by the force generated from the untilting motion. The change in angle $\theta$ as per the studies of [4] is around $15^{\circ}$. So the post power stroke value of $\theta$ was determined to be $111^{\circ}$ and thus the pre power stroke value of $\theta$ was approximated to be $96^{\circ}$. When the power stroke is complete the head tries to retrace its trajectory; however the binding of the myosin head to actin monomer places constraints on this from happening. As a consequence the rotation of the myosin head takes place when its still bound to the actin filament. This state is termed as rigor state. There is no nucleotide bound to the myosin in rigor state. The next cycle can take place only after another molecule of ATP binds and supplies the energy required to break the actomyosin interactions. This energy detaches the myosin from actin and readies it for hydrolysis.

\subsection{Brownian motion}

Cytoskeletal filaments i.e. Actin when dispersed in liquid constantly undergo random movements. This phenomenon was first observed by botanist Brown and then later explained by Einstein and Smouluchowski in terms of thermally excited collisions. The diffusion coefficients depend on the temperature $\mathrm{T}$ and the viscosity of the liquid which reflects the balance between thermal fluctuations and viscous dissipation. These diffusive processes are commonly found in biological systems and particularly in biological cells. 


\subsubsection{History of Brownian motion}

Brownian motion was first described by the botanist Robert Brown in 1828. The Brownian motion was first perceived as uninterrupted and irregular swarming motion. The investigation on Brownian motion was further carried by Regnault (1858), Weiner (1863), Jevons (1870), and Delsaux (1877) before Einstein. Gouy (1888) observed that the motion is livelier when the viscosity of the liquid is smaller. The motion was ascribed to the thermal molecular motion of the liquid. Einstein in 1905 was the first to formulate a correct picture of the entire problem.

\subsection{Theory and Assumptions}

The irregular movements of the particles arising from the thermal molecular movement give rise to diffusion. The assumptions in this case is that (i) that each particle executes a movement that is independent of movement of all other particles (ii) the movement of of one and same particle after different intervals of time must be considered as mutually independent processes, if we think these intervals are not too small.[6]

The expression for the mean displacement of the Brownian particle is as given

$$
\lambda=\sqrt{2 D t}
$$

where $\lambda$ is the mean displacement, $D$ is the diffusion constant, and $t$ the time. The mean displacement is therefore proportional to square root of time. This is a typical characteristic of Brownian motion. The diffusion is a result of the irregular movement of particles produced by the thermal molecular movement of the liquid. The expression for $D$ is as follows. 


$$
D=\frac{R T}{N} B
$$

$R$ is the universal gas constant, $T$ is the temperature in Kelvin, $N$ is Avogadro number and $B$ is the mobility of the liquid. The mobility is expressed as follows

$$
B=\frac{1}{6 \pi \kappa \rho}
$$

wherein $\kappa$ is the coefficient of viscosity of the liquid, $\rho$ is the radius of the Brownian particle which is assumed to be spherical. The kinetic energy of the motion of the particle is independent of the size and nature of the particle and independent of the nature of its environment, e.g. of the liquid in which the particle is suspended. The velocity and direction of the motion of the particle is greatly altered in a very short time and in a greatly irregular manner [6]. From hydrodynamics we can say that if the velocity is imparted to a particle in brownian motion by an impulsive force, it will die away quickly. To maintain the average velocity the particle must experience impulses from liquid molecules whose direction and magnitude are random. The fluctuations in the collisions with the molecules of the surrounding liquid maintain the perpetual motion of the brownian particles in the liquid. In absence of any external force the Langevin equation of the brownian motion can be written as

$$
m \frac{d v}{d t}=-6 \pi \eta v r+F(t)
$$

where $\eta$ is the viscosity of the liquid, $r$ is the radius of the brownian particle and $v$ the velocity of the particle. The above equation divides the influence of the liquid medium into two parts: 
- A systematic part $-6 \pi \eta v r$ which represents the dynamic friction experienced by the brownian particle

- A fluctuating force which, $F(t)$, which is characteristic of brownian motion., The assumption in this case are (i) $F(t)$ is independent of $v$ and $\langle F(t)\rangle=0$ (ii) $F(t)$ is extremely rapid when compare to the variations of $v$.

\subsubsection{Brownian motion in Velocity Space: Fokker Planck Equation}

Previous theory dealt with the brownian in co-ordinate space, i.e. it concerns the time development of distribution of suspended particles in terms of $f(x, t)$, or the probability of finding a particle at the position $x$ at a time $t$. In the velocity space approach the probability is $f(v, t)$. A particle has the velocity $v$ at a time $t$. The Fokker Planck equation for the velocity space method is as follows [6].

$$
\frac{\partial f(v, t)}{\partial t}=-\frac{\partial}{\partial v} M_{1}(v) f(v, t)+\frac{\partial^{2}}{\partial v^{2}} M_{2}(v) f(v, t)
$$

$M_{1}(v)$ and $M_{2}(v)$ are called the drift and diffusion terms respectively. $M_{2}(v)$ is the diffusion in the velocity space and is not to be confused with the $D$ of the co-ordinate space. The $M_{1}$ and $M_{2}$ here are model specific, for a particle in brownian motion they are as follows.[6]

$$
\begin{aligned}
& M_{1}(v)=-\alpha v \\
& M_{2}(v)=D=\frac{\alpha k_{B} T}{m}
\end{aligned}
$$

A more quantitative approach towards the random motion of biomolecular motors is beyond the scope of this report. Further discussion on this topic can be found in the 
journal paper of [7].The next chapter shall describe the methods employed to fabricate the electrode chip.

\subsection{Graphical description of Brownian motion in 2 and 3-Dimensions}

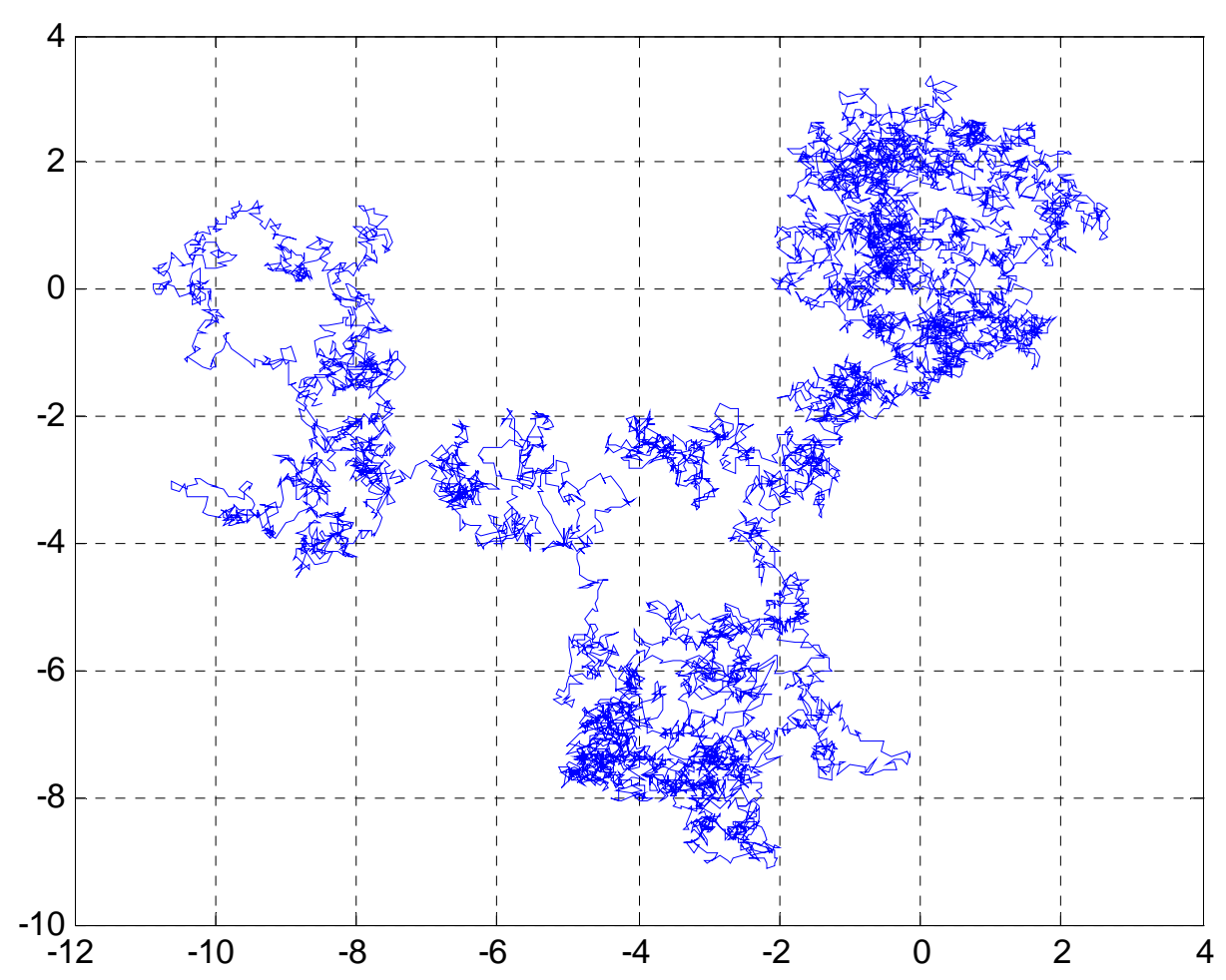

Figure 7 Simulation of Brownian motion in 2-D space

The Brownian motion was simulated in MATLAB. The number of steps or the numbers of collisions for the simulation were assumed to be 10,000 . The initial position of the particle was assumed to be the origin. The particle undergoing Brownian motion changes its position in space due to the collisions. It is assumed that the particle changes its coordinates every time when it collides. The changes in the positions were recorded and 
plotted. The motion was simulated and plotted for $2 \& 3$ dimensions. The programs that generate the above plots are available for reference in the appendix section.

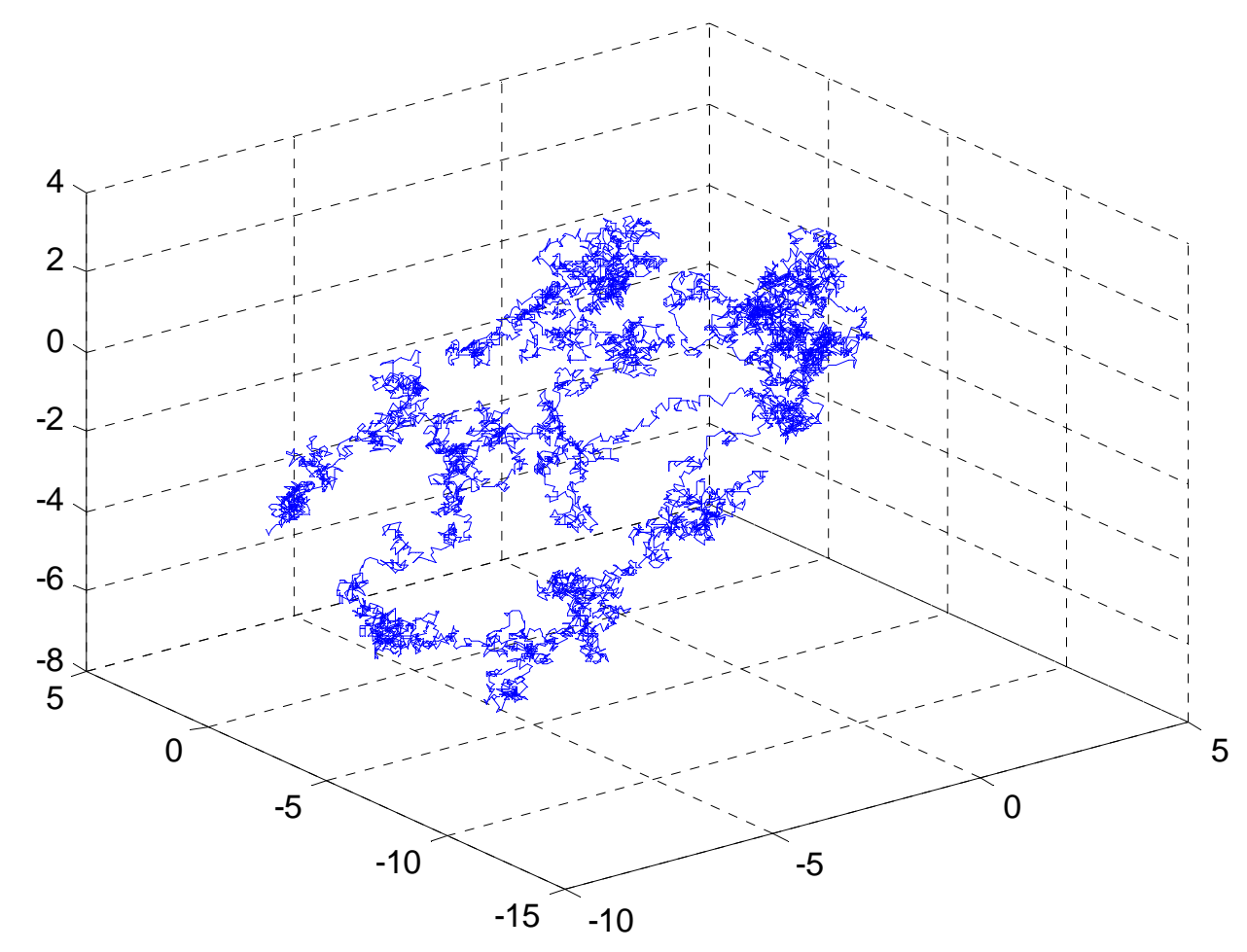

Figure 8 Simulation of Brownian motion in 3-d space 


\section{Chapter 3. Modeling and Simulations}

\subsection{Simulation conditions}

The design of the electrode structures, both large electrode structures and the microelectrode structure was primarily based on simulated observations of electric fields between the electrode structures. The simulations were simulated using the Electrostatic simulation and analysis tool of Ansoft MAXWELL. The simulations tried to replicate the exact conditions of the experiment. The features of the experiment that were included in the simulations were

- The thickness of silicon dioxide deposition.

- The thickness of the collodion membrane.

- The conductivity of the buffer solution.

- The height of the buffer solution in the flow cell.

- The height of metal electrodes.

- The gap between the electrodes.

The simulations were performed for different ranges of voltages, different widths between the electrode structures, both macro and micro electrodes. The simulations of the large electrodes were of 2-D and 3-D. The voltages of the simulations of microelectrodes were determined based on the reference [3]. The paper showed the field of response of myosin-actin motility to be in between $500 \mathrm{~V} / \mathrm{m}-5000 \mathrm{~V} / \mathrm{m}$. The macro electrodes were considered as more appropriate to experiment with varying field strengths due to the different applied voltages. 


\subsection{2-D Simulations of Chrome Macro electrode structures}

The simulations were carried out for different gaps between the electrodes, the gaps primarily being $1 \mathrm{~mm}, 2 \mathrm{~mm}, 5 \mathrm{~mm}$ and $10 \mathrm{~mm}$. The voltages for which the simulations were carried out were $3 \mathrm{~V}, 5 \mathrm{~V}, 7 \mathrm{~V}$. The electrodes in the macro structures were made of chrome. The height of the electrodes was $200 \mathrm{~nm}$. The thickness of silicon dioxide deposited through PECVD is $0.5 \mathrm{~m}$. The simulations are as follows

\subsubsection{Simulations for the gap of $10 \mathrm{~mm}$}

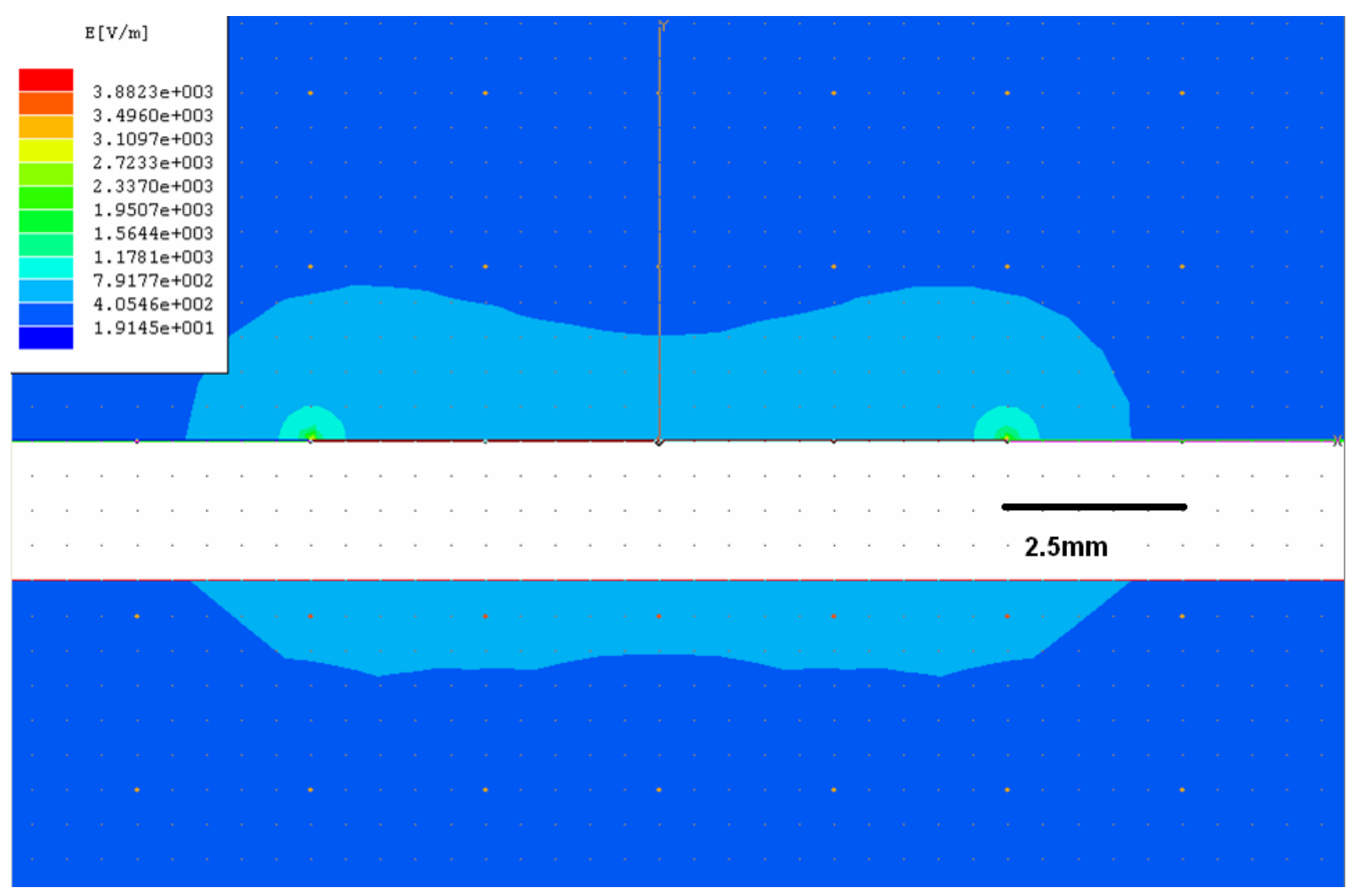

Figure 9 10mm gap Applied Voltage $=3 \mathrm{~V}$ 


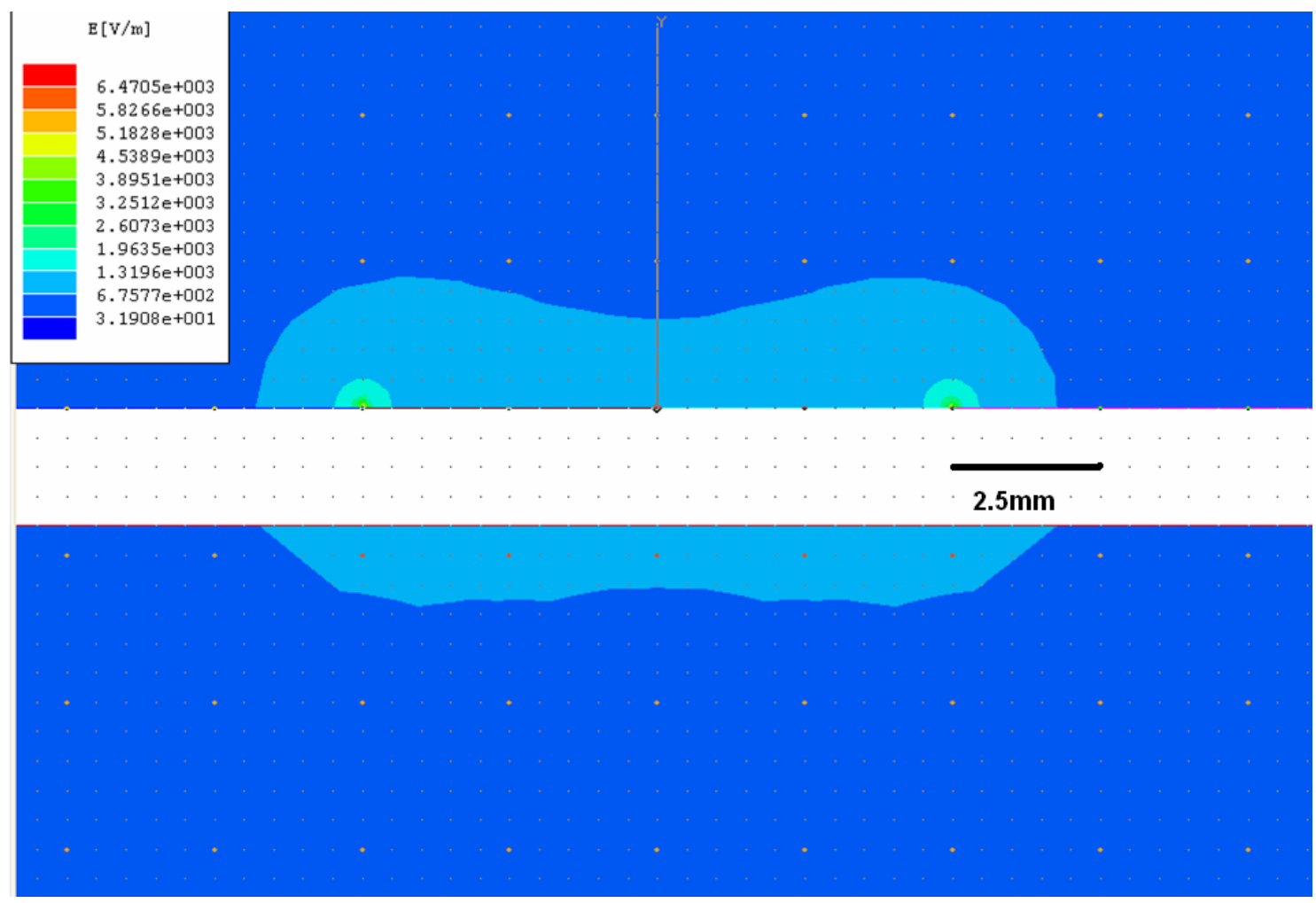

Figure $1010 \mathrm{~mm}$ Gap Applied Voltage $=5 \mathrm{~V}$.

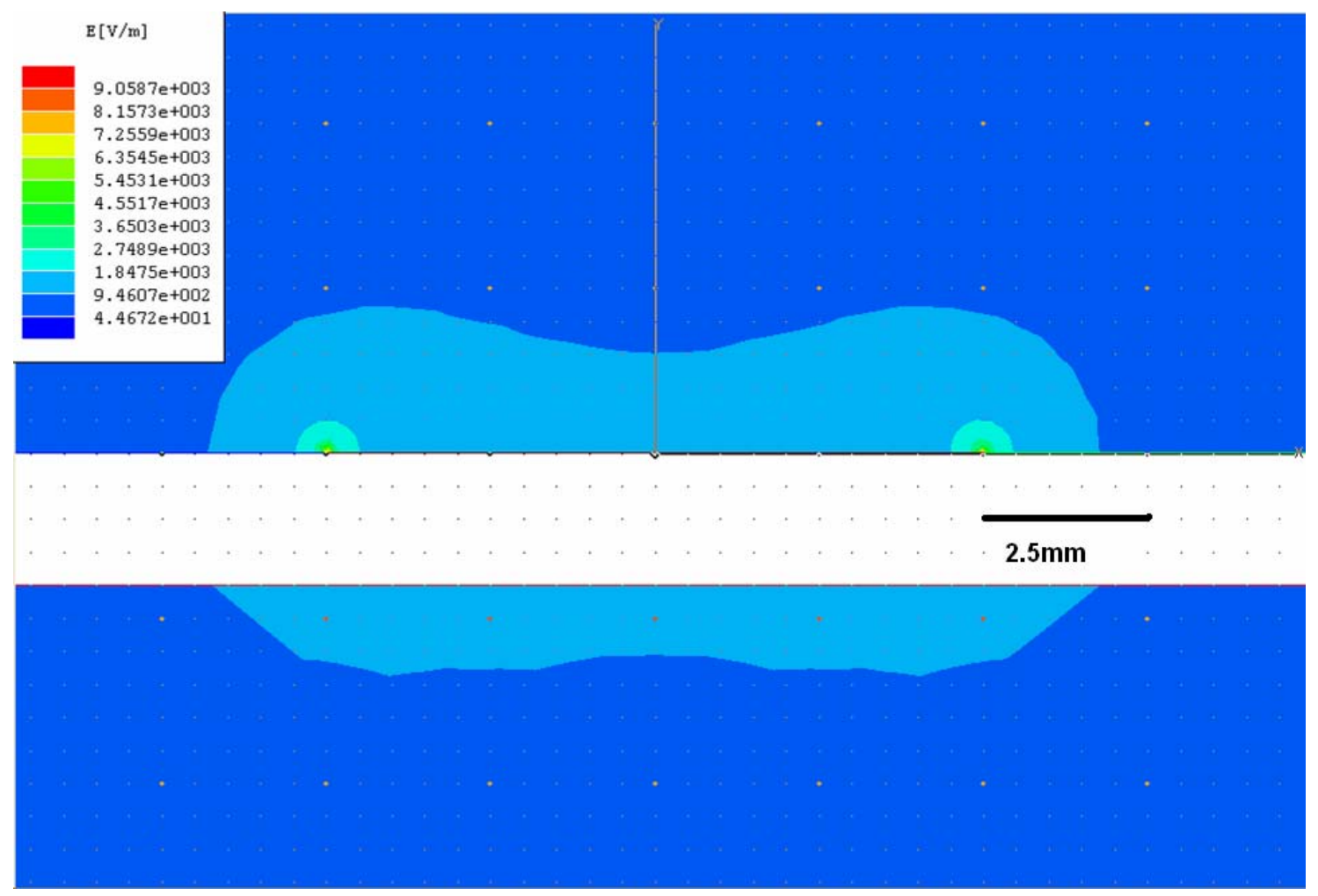

Figure 11 10mm Gap Applied Voltage $=7 \mathrm{~V}$ 


\subsubsection{Simulations for the Gap of $5 \mathrm{~mm}$}

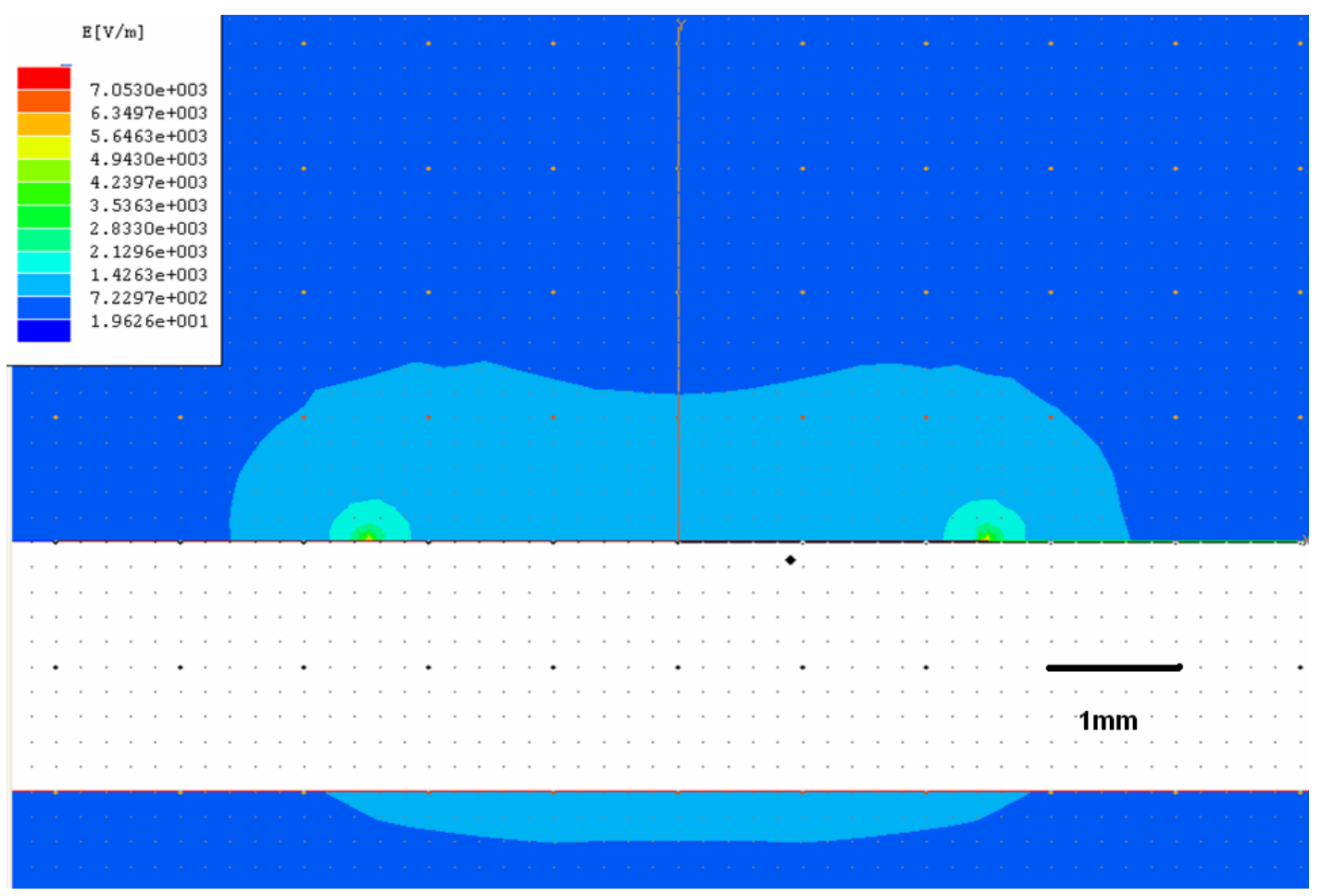

Figure $125 \mathrm{~mm}$ Gap Applied Voltage $=3 \mathrm{~V}$

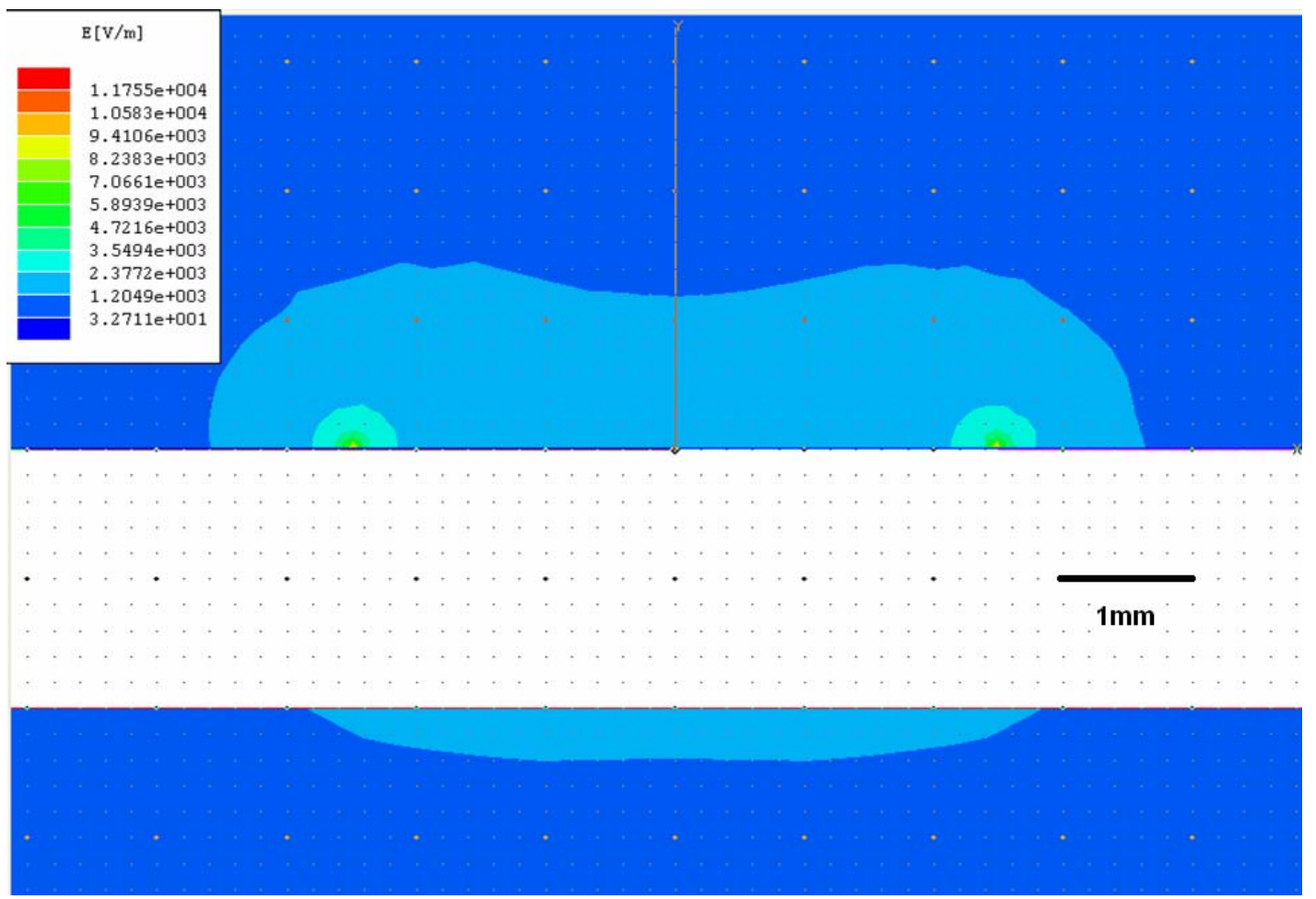

Figure 13 5mm Gap Applied Voltage $=5 \mathrm{~V}$ 


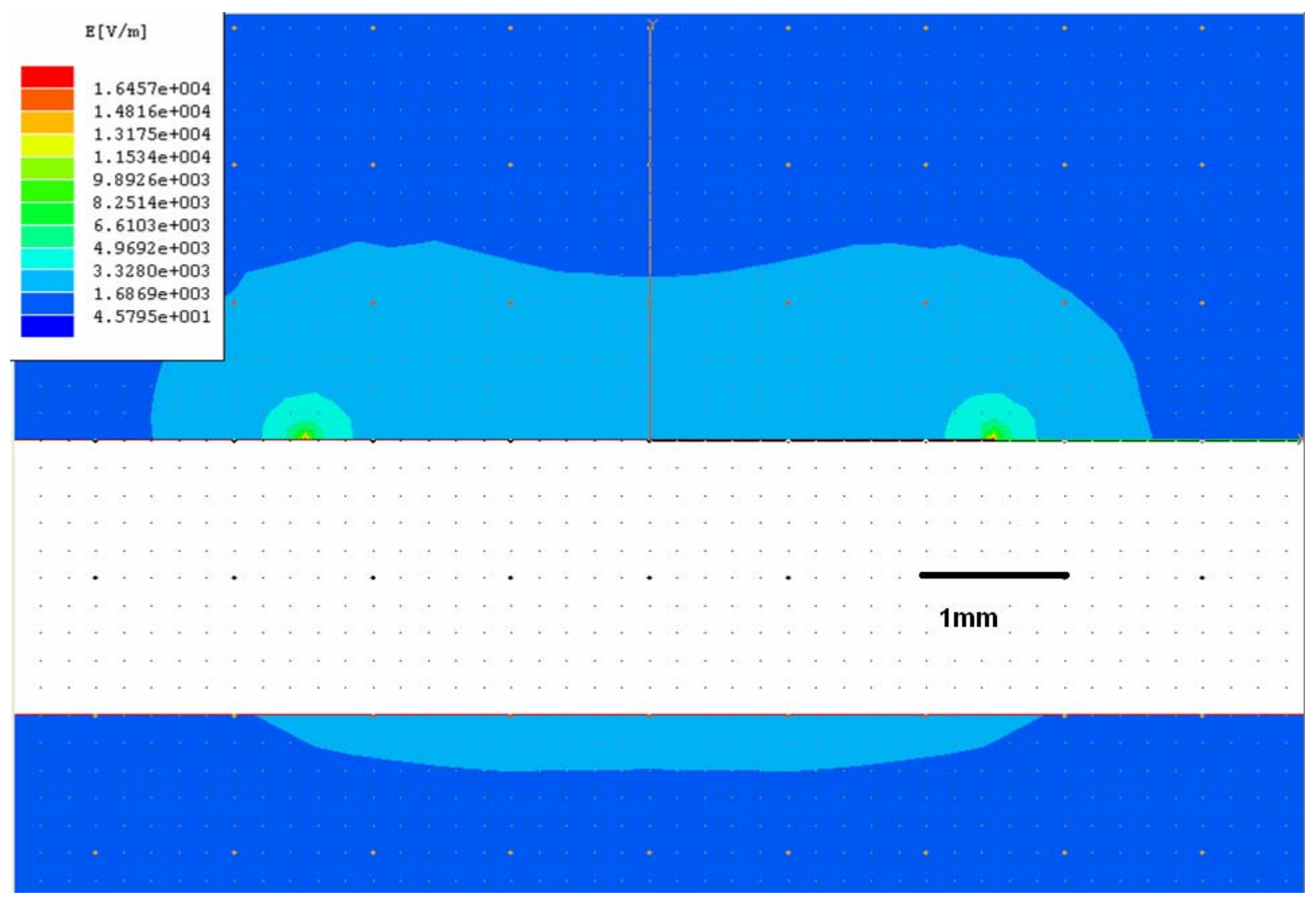

Figure $145 \mathrm{~mm}$ gap Applied Voltage $=7 \mathrm{~V}$.

3.2.3. Simulations for the gap of $2 \mathrm{~mm}$

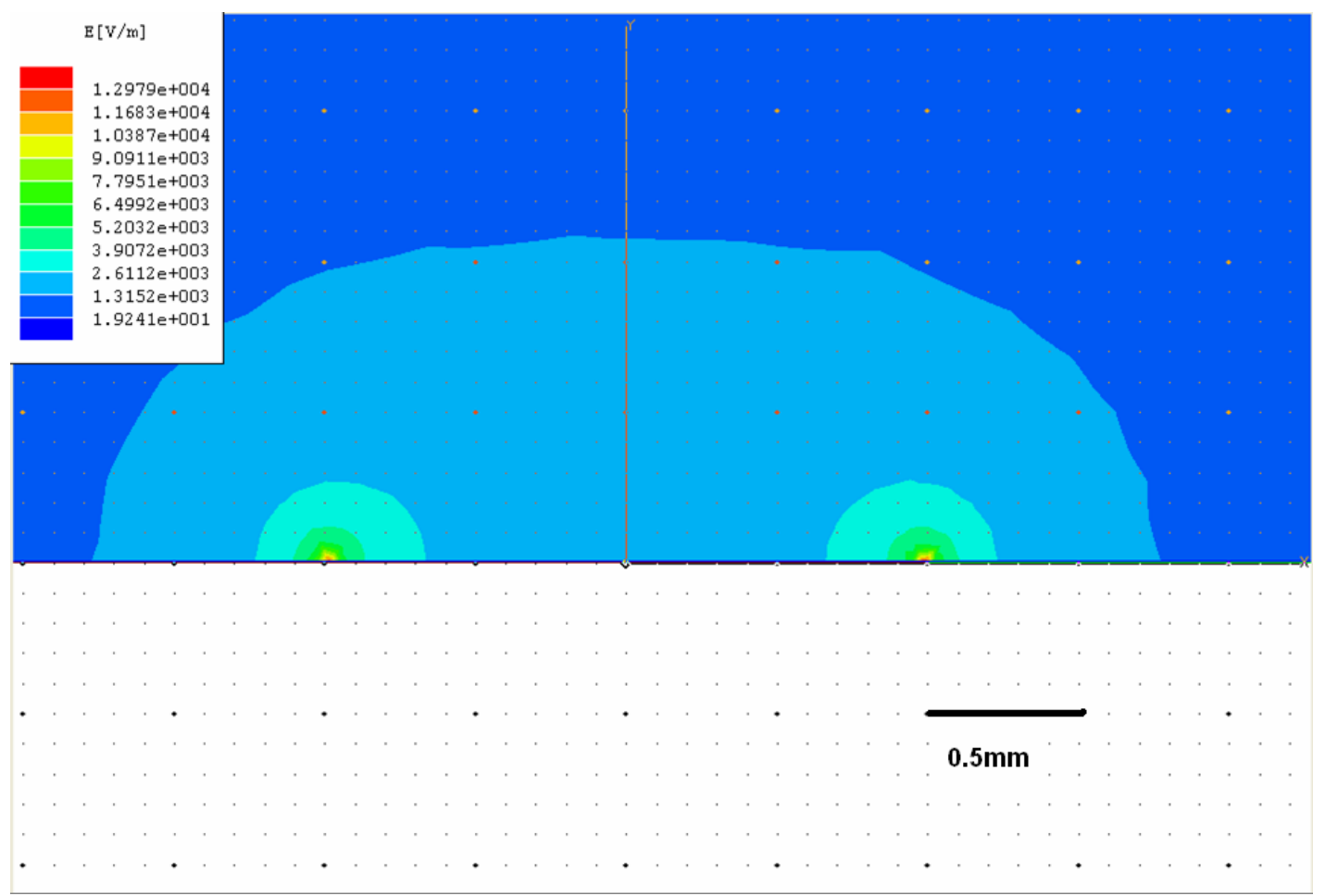

Figure $152 \mathrm{~mm}$ Gap Applied Voltage $=3 \mathrm{~V}$ 


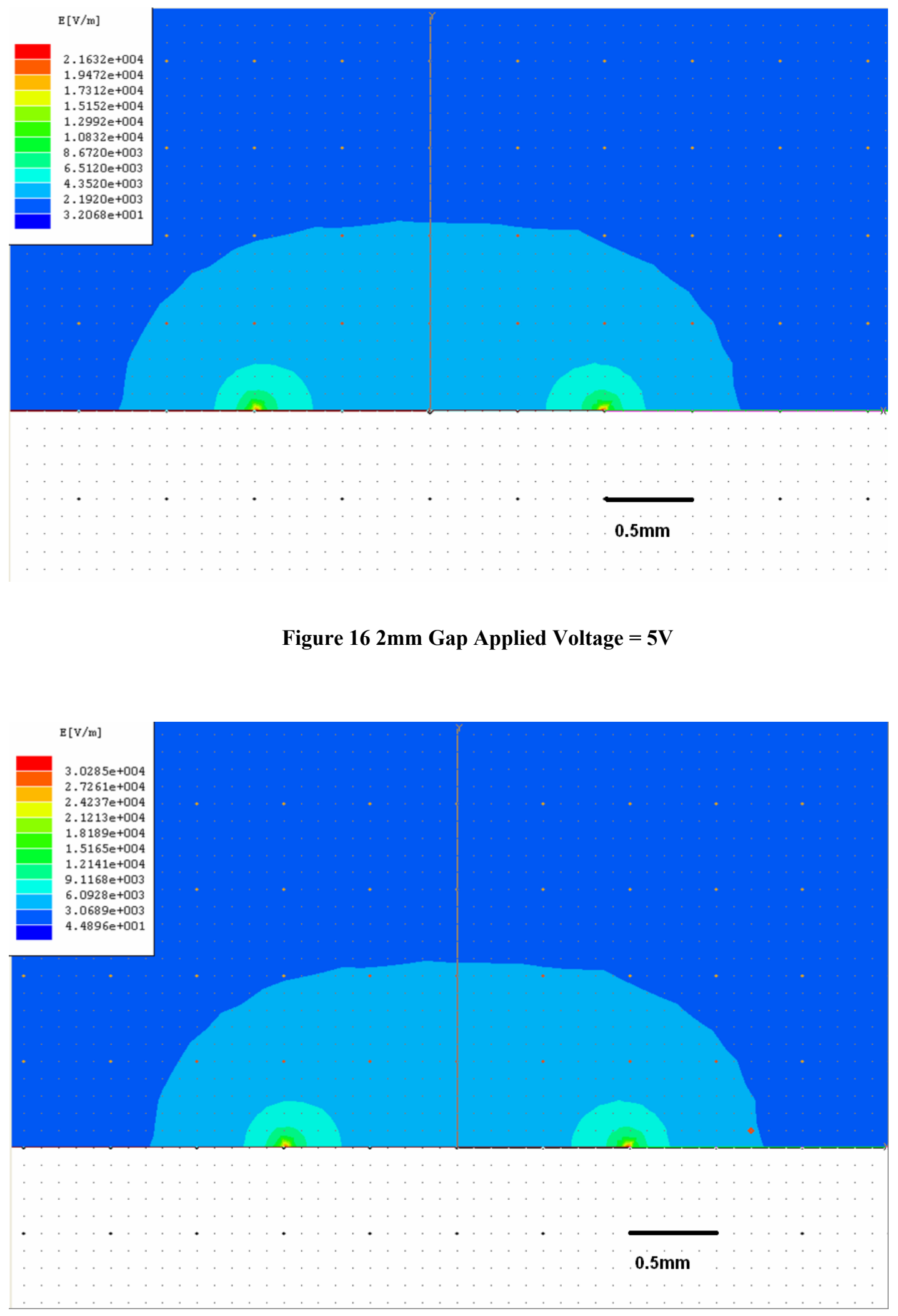

Figure 17 2mm Gap Applied Voltage $=7 \mathrm{~V}$ 


\subsubsection{Simulations for the gap of $1 \mathrm{~mm}$.}

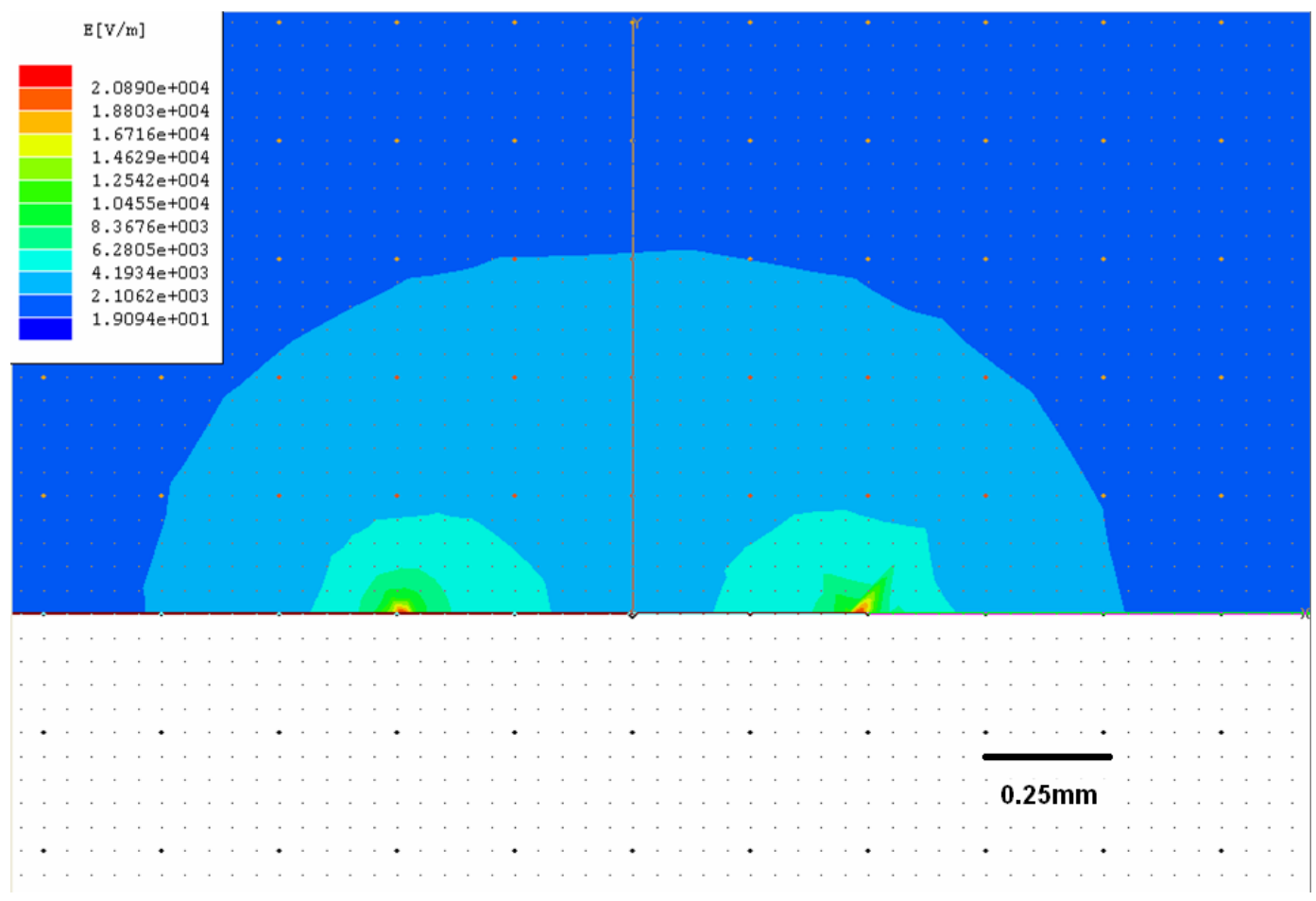

Figure $181 \mathrm{~mm}$ Gap Applied Voltage $=3 \mathrm{~V}$

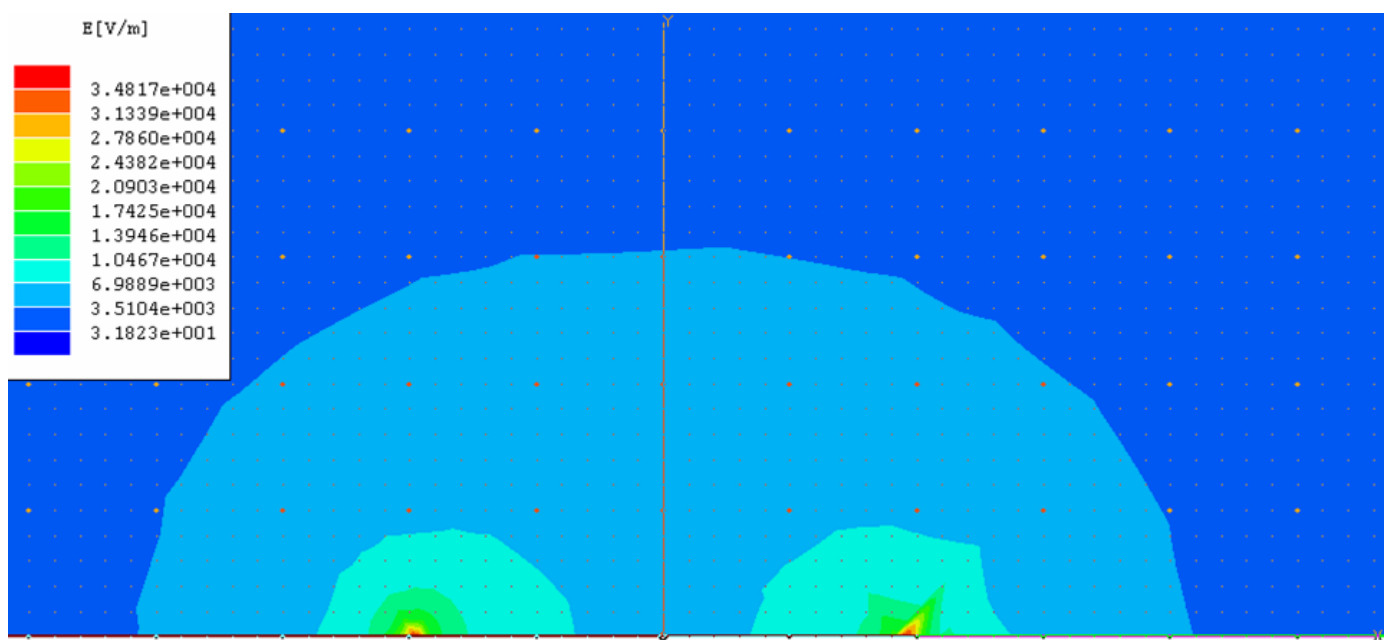

Figure $191 \mathrm{~mm}$ Gap Applied Voltage $=5 \mathrm{~V}$ 


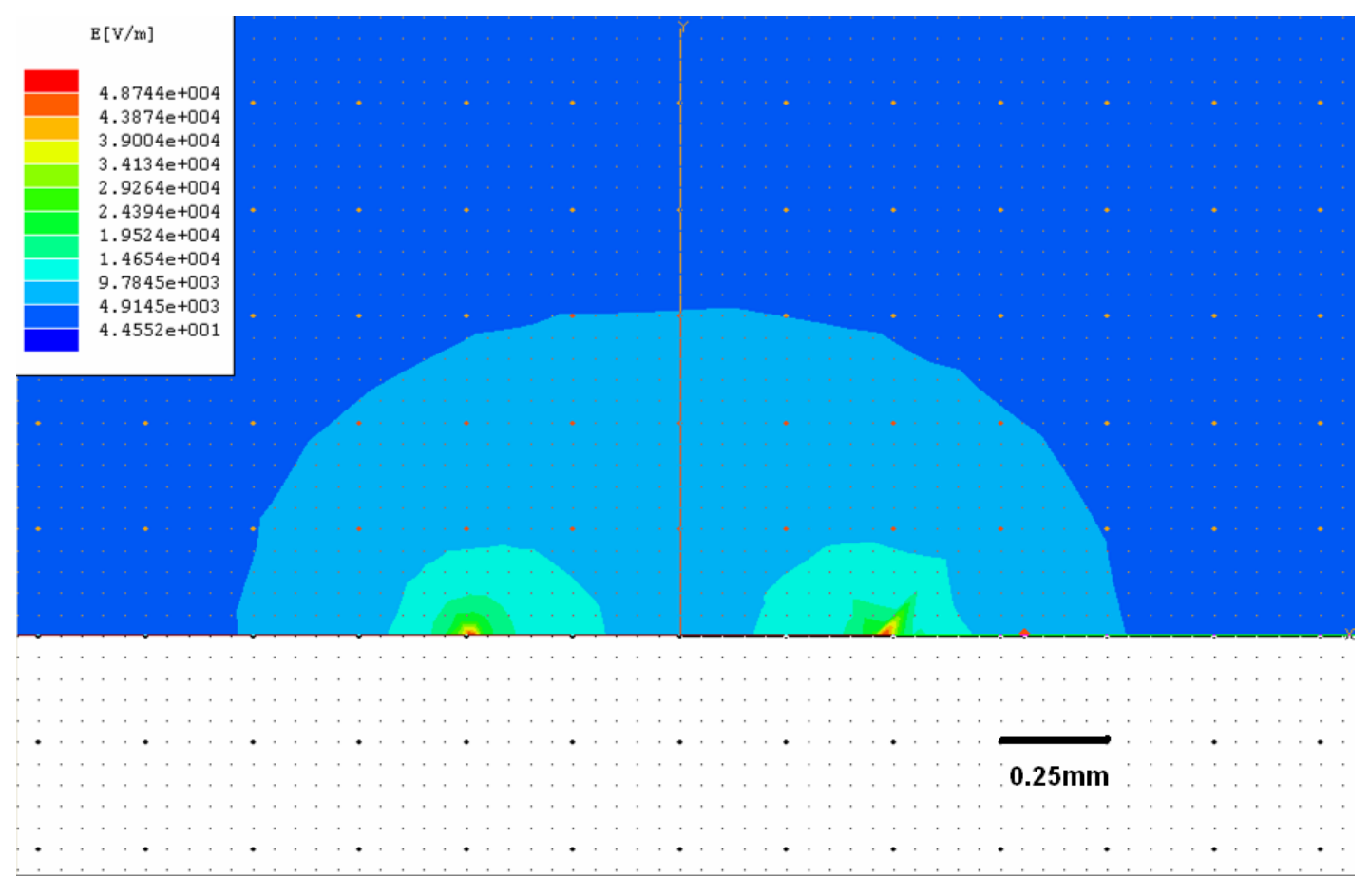

Figure $201 \mathrm{~mm}$ gap Applied voltage $=7 \mathrm{~V}$

\subsection{3-D Simulations of Chrome Macro electrode structures.}

The three dimensional simulations were carried out using the 3-D Electrostatic Simulation tool of MAXWELL. Care was taken to prevent the overlapping of the layers of the designated materials while trying to draw the 3-D structure. Overlapping structures prevent the tool from simulating the simulations. The simulations were once again carried out for the gaps of $10 \mathrm{~mm}, 5 \mathrm{~mm}, 2 \mathrm{~mm}$ and $1 \mathrm{~mm}$ gaps with voltages of $3 \mathrm{~V}, 5 \mathrm{Vand} 7 \mathrm{~V}$. The 3-D simulations were performed to compliment the 2-D simulations and to observe the spatial distribution of field strengths. 


\subsubsection{3-D simulations for gap of $10 \mathrm{~mm}$}

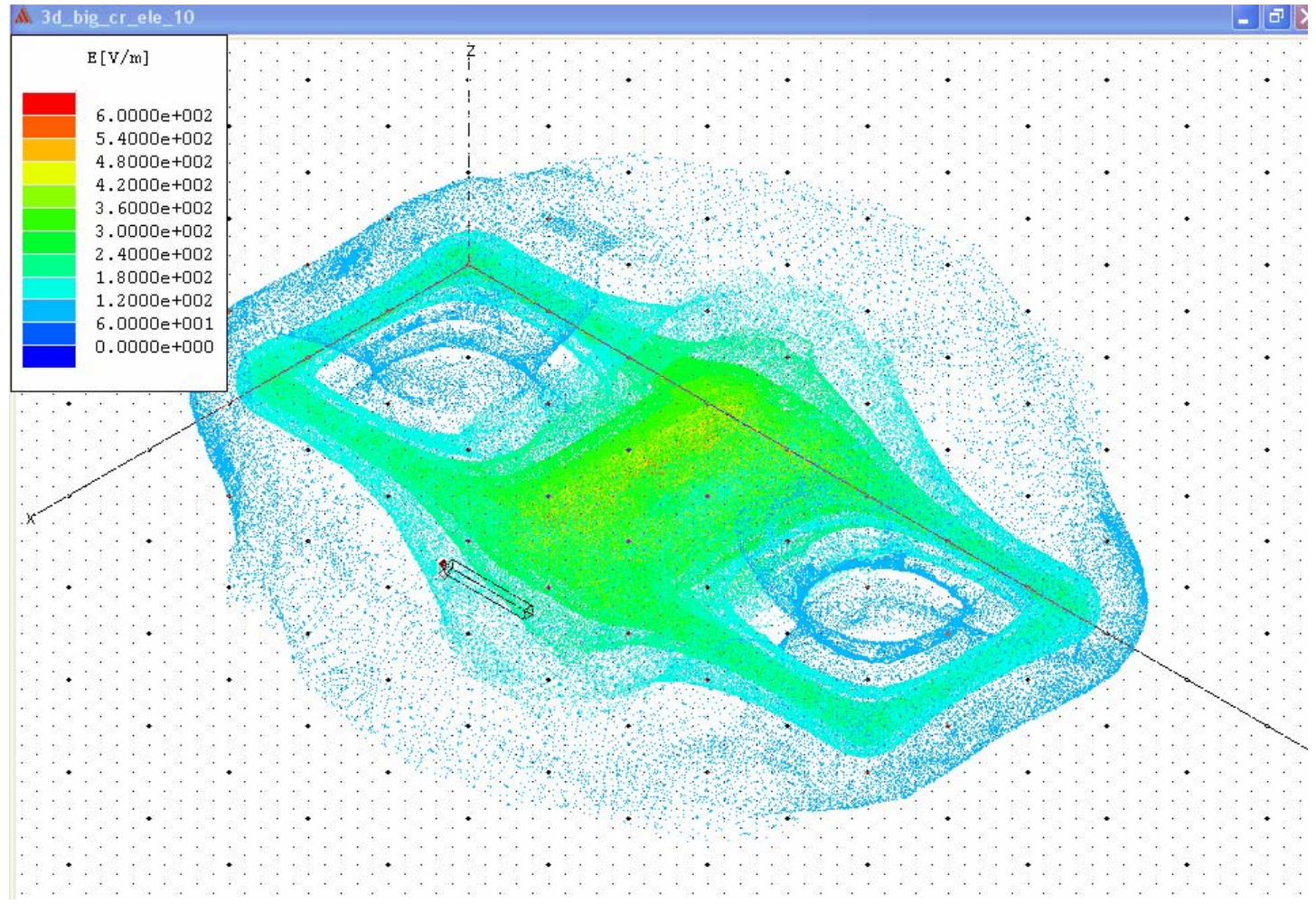

Figure 21 3-d 10mm Gap AppliedVoltage $=3 \mathrm{~V}$

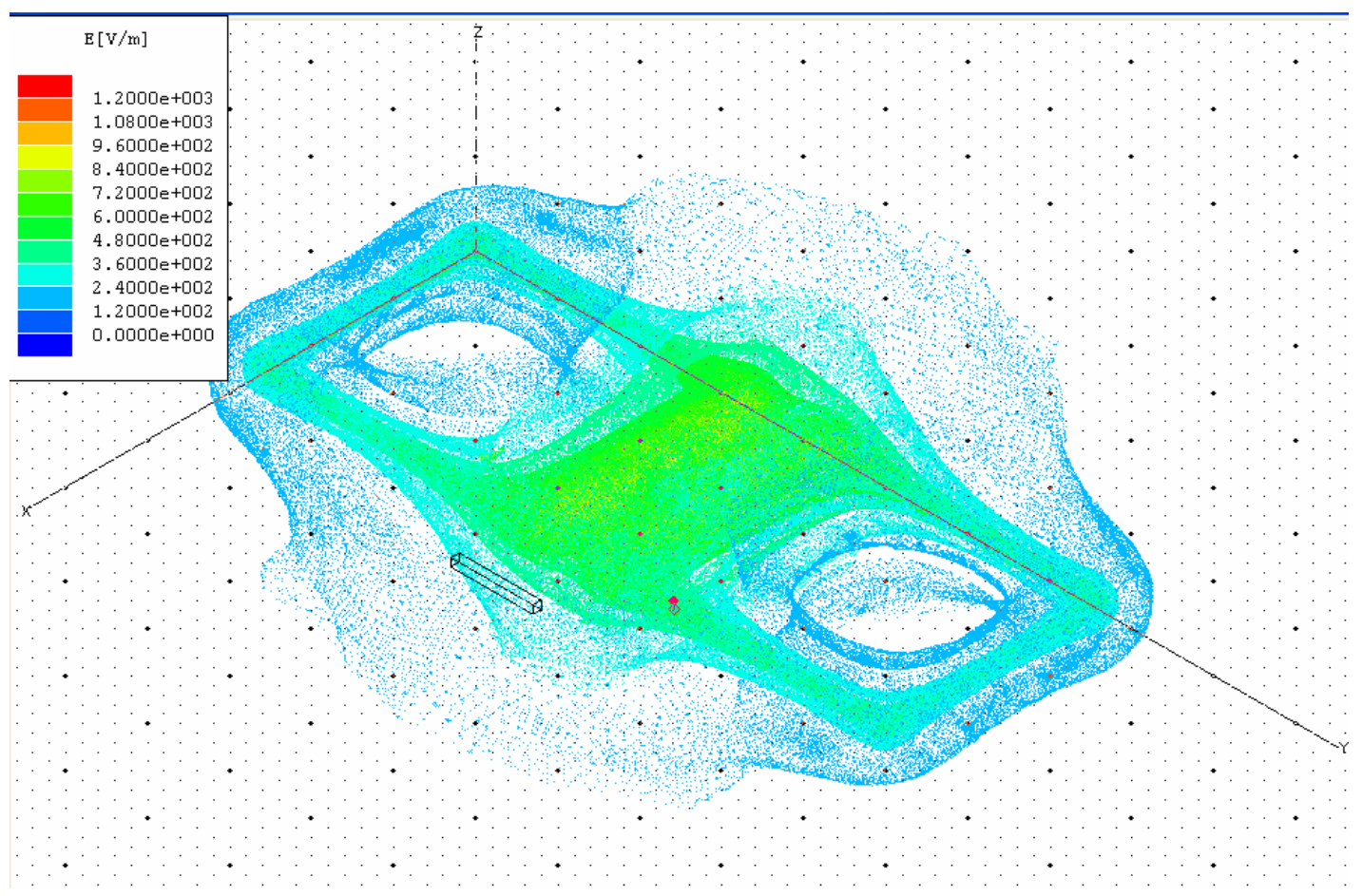

Figure 22 3-d 10mm Gap Applied Voltage $=5 \mathrm{~V}$ 


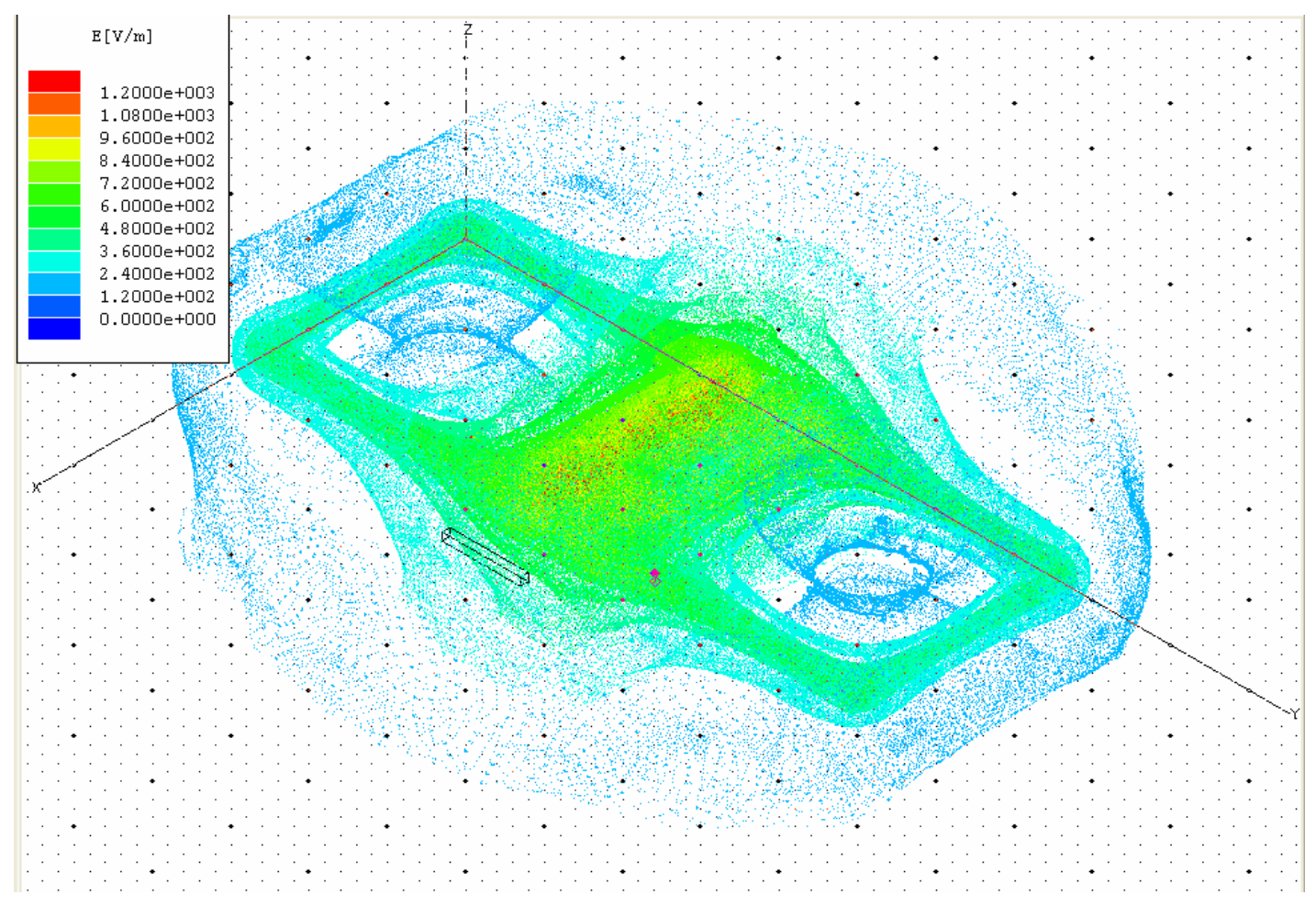

Figure 23 3-d $10 \mathrm{~mm}$ gap Applied $=7 \mathrm{~V}$

\subsubsection{3-D Simulations for a gap of $5 \mathrm{~mm}$}

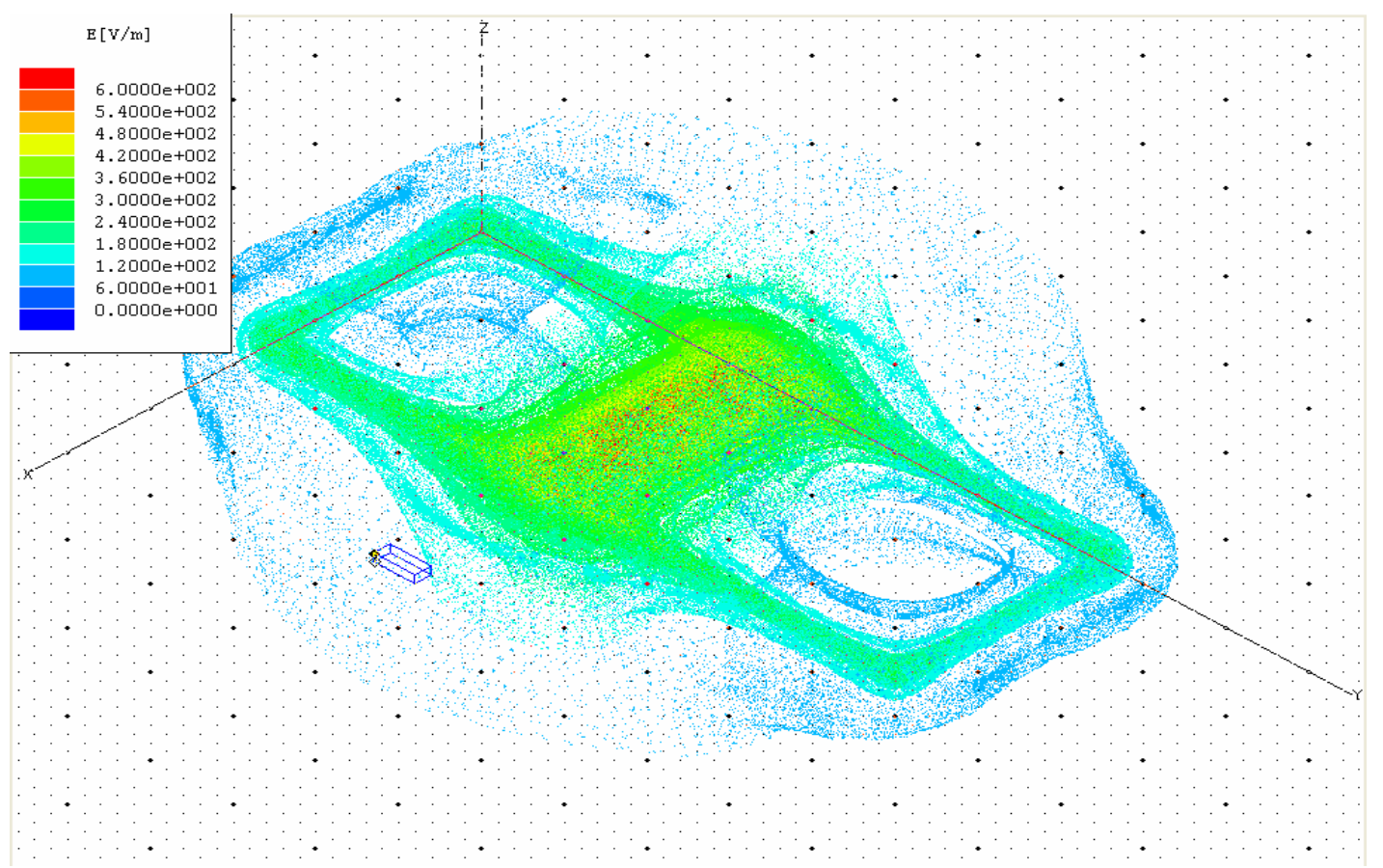

Figure 24 3-d 5mm Gap AppliedVoltage $=3 \mathrm{~V}$ 


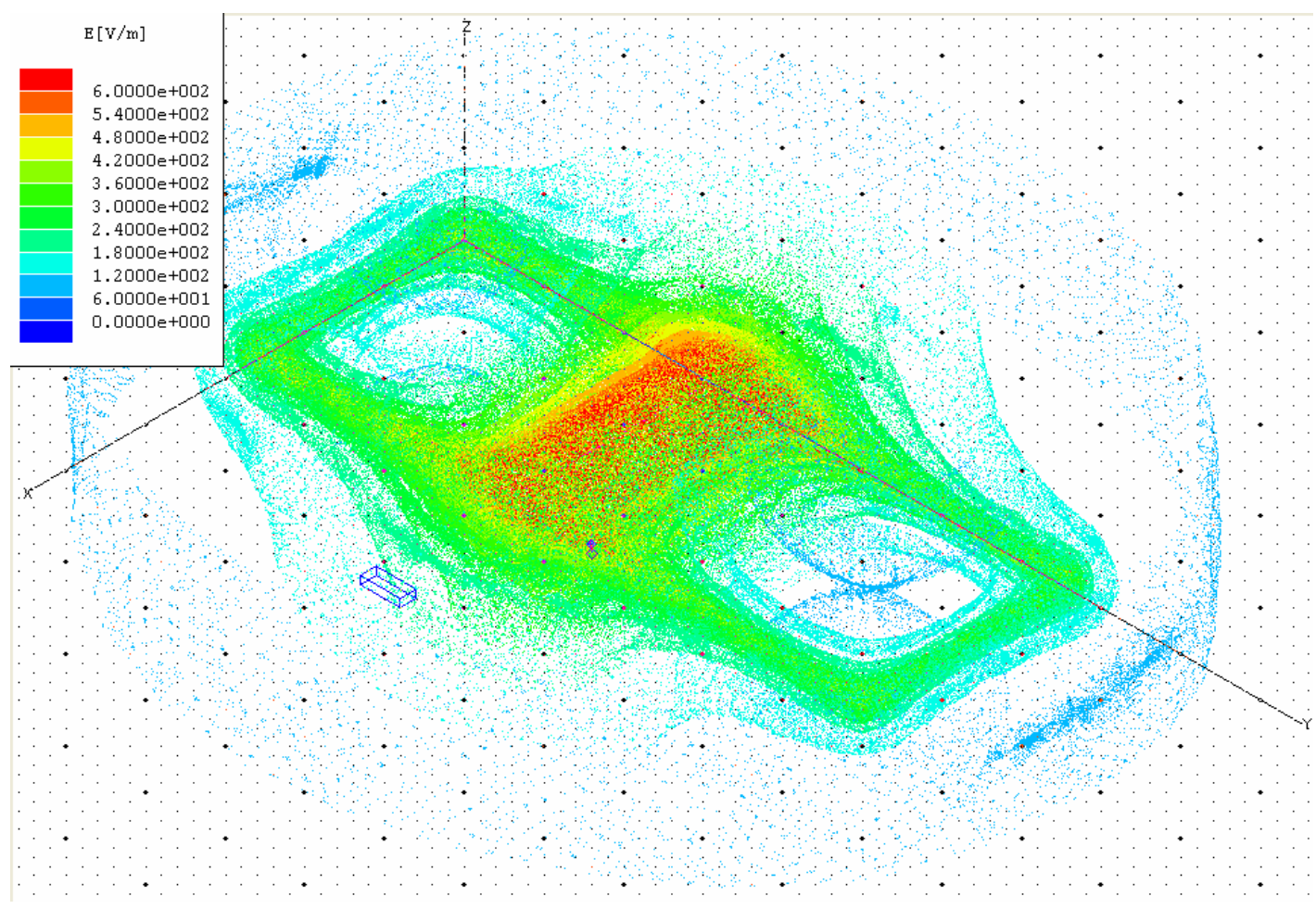

Figure 25 3-d 5mm gap Applied Voltage $=5 \mathrm{~V}$

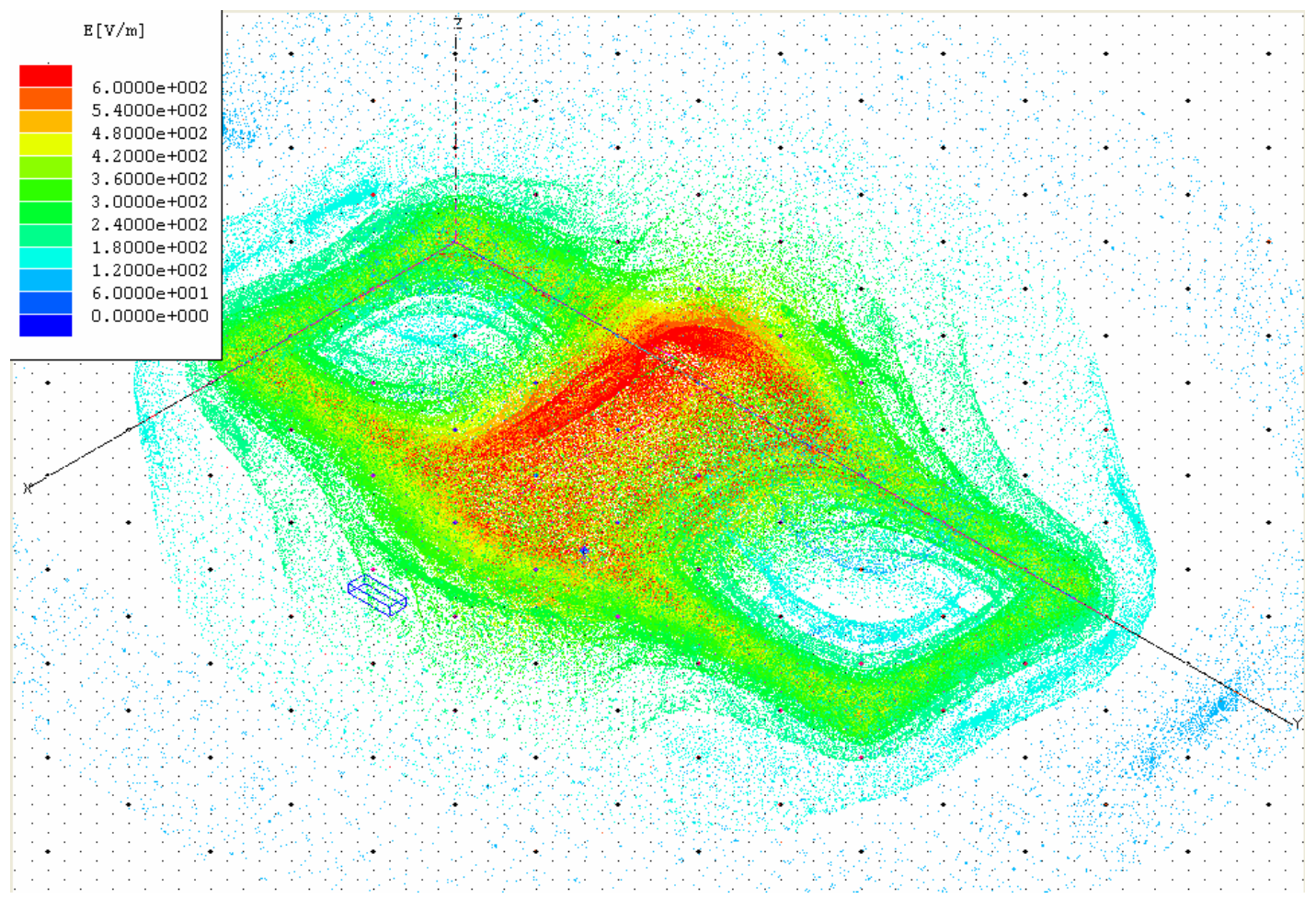

Figure 26 3-d 5mm gapApplied Voltage $=7 \mathrm{~V}$ 


\subsubsection{3-D Simulation for a gap of $2 \mathrm{~mm}$ gap}

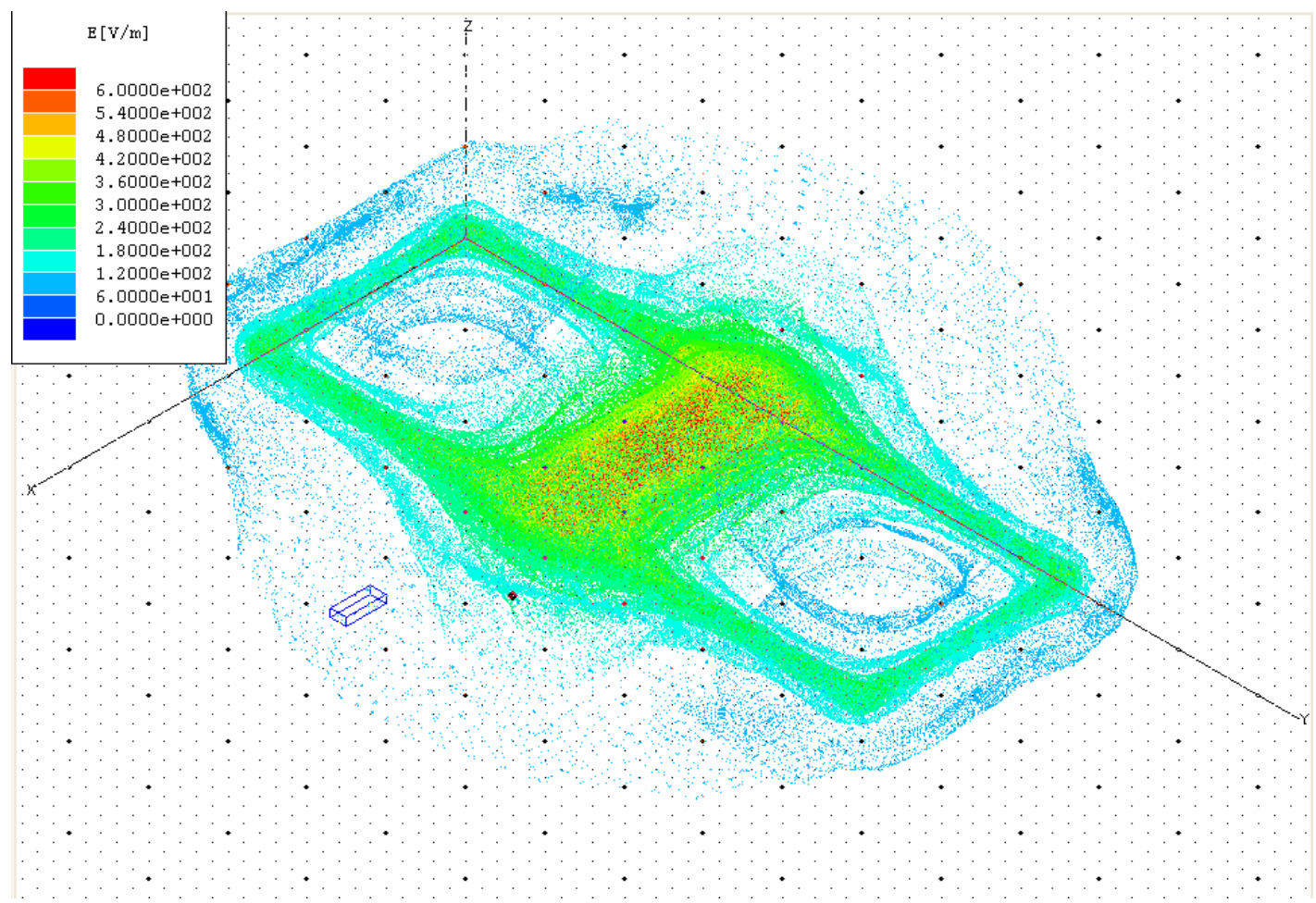

Figure 27 3-d 2mm gap AppliedVoltage $=3 \mathrm{~V}$

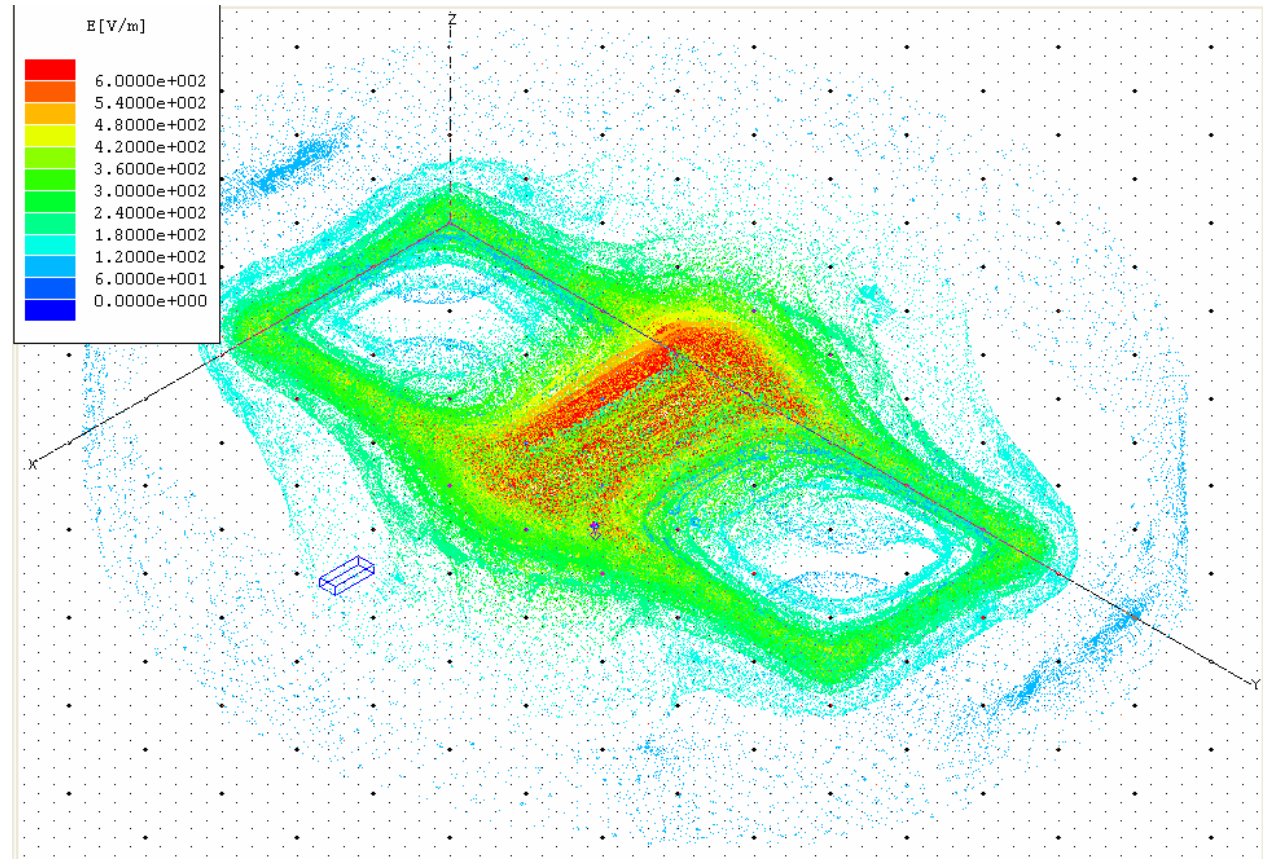

Figure 28 3-d 2mm gap Applied Voltage $=5 \mathrm{~V}$ 


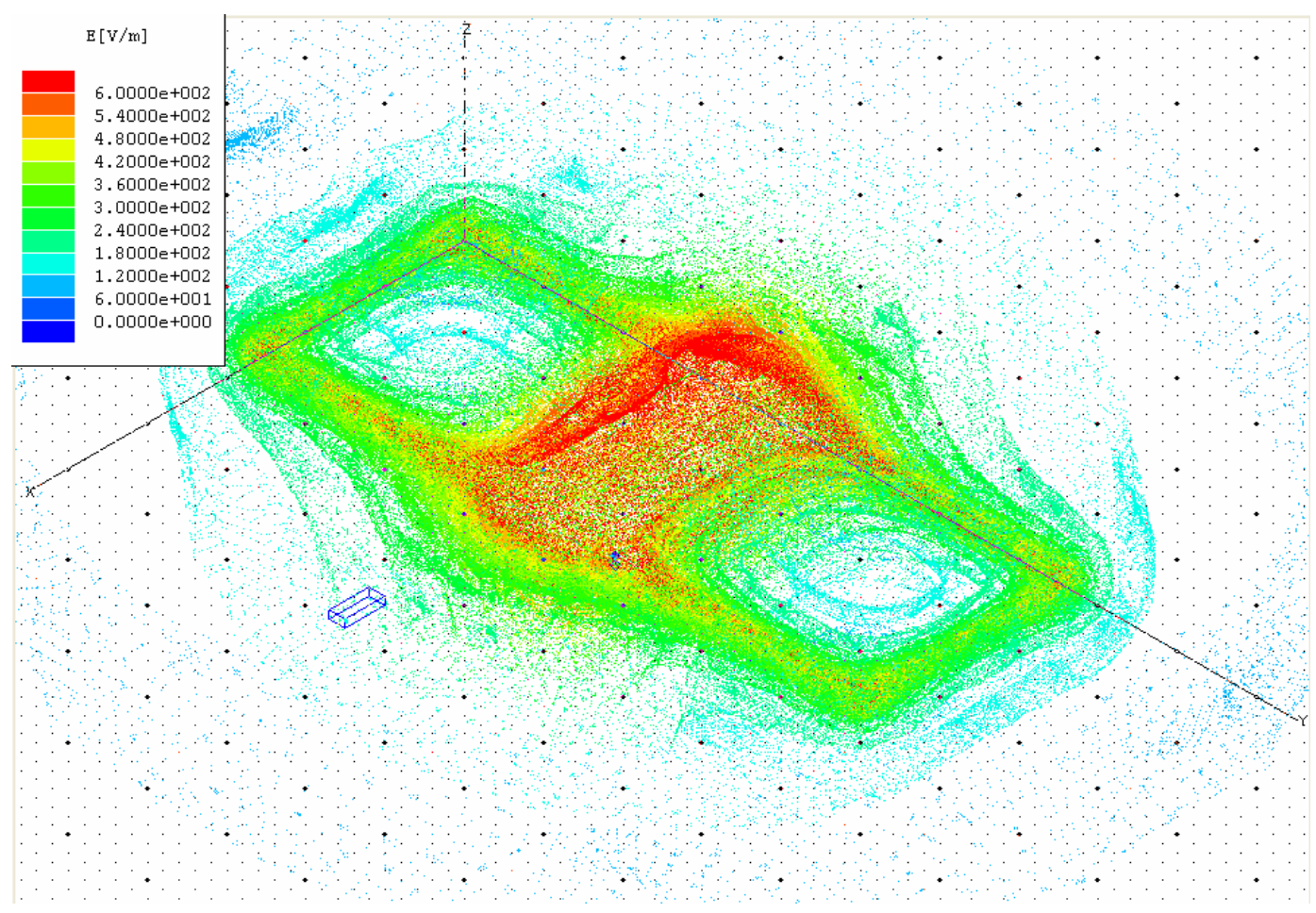

Figure 29 3-d 2mm gap Applied Voltage $=7 \mathrm{~V}$

\subsubsection{3-D Simulation for a gap of $1 \mathrm{~mm}$}

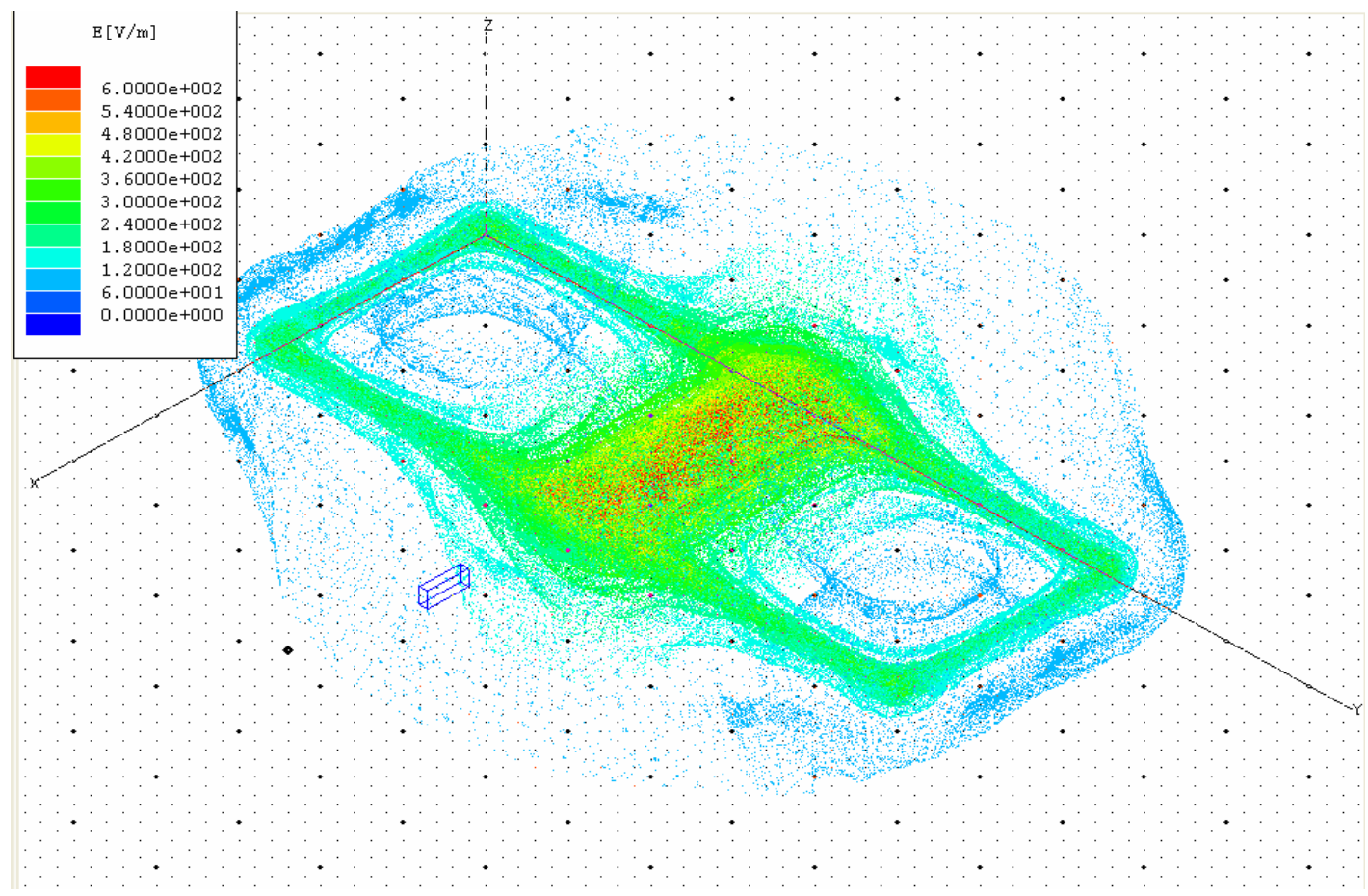

Figure 30 3-d 1mm gap AppliedVoltage $=3 \mathrm{~V}$ 


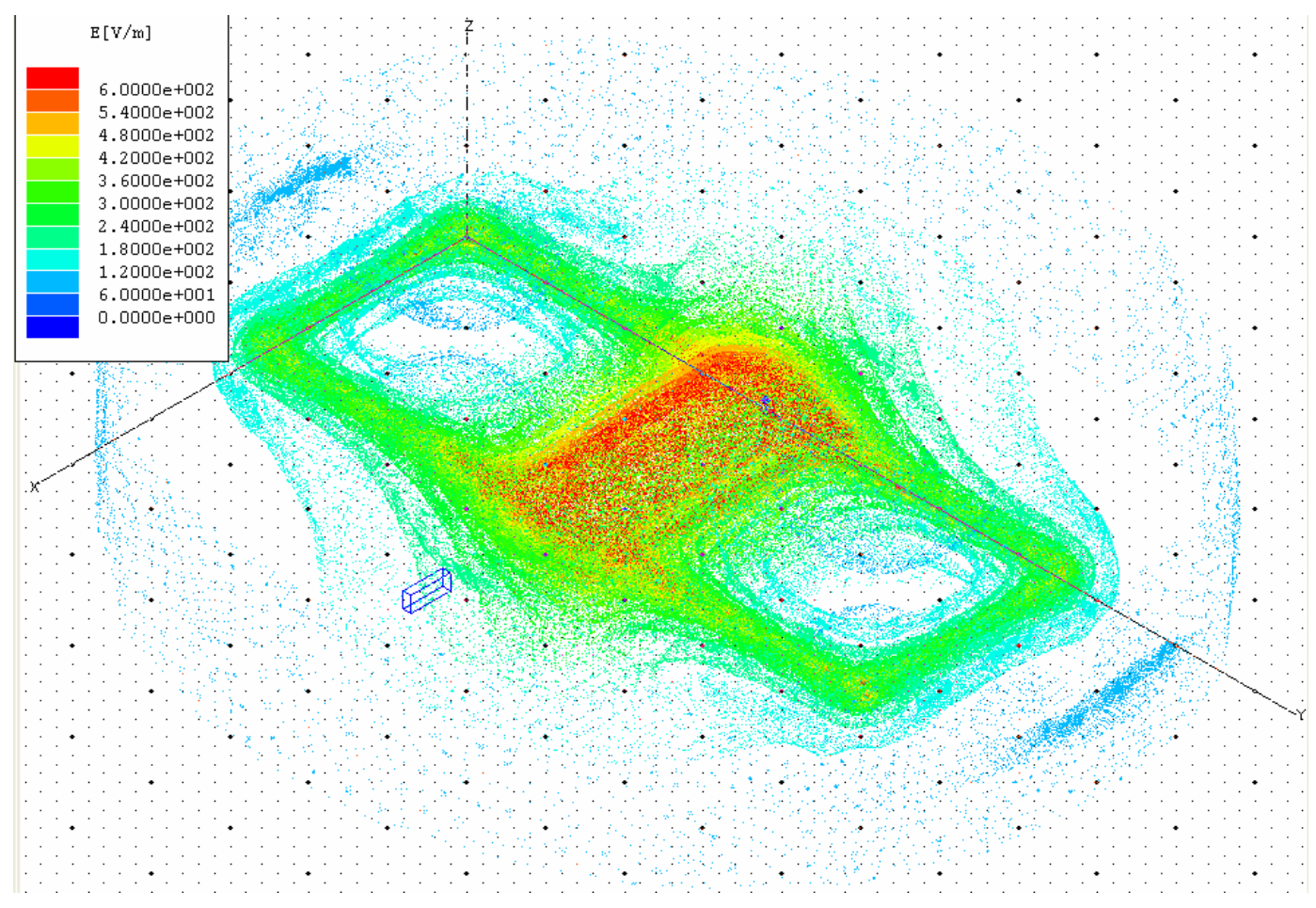

Figure 31 3-d $1 \mathrm{~mm}$ gap AppliedVoltage $=\mathbf{5} \mathrm{V}$

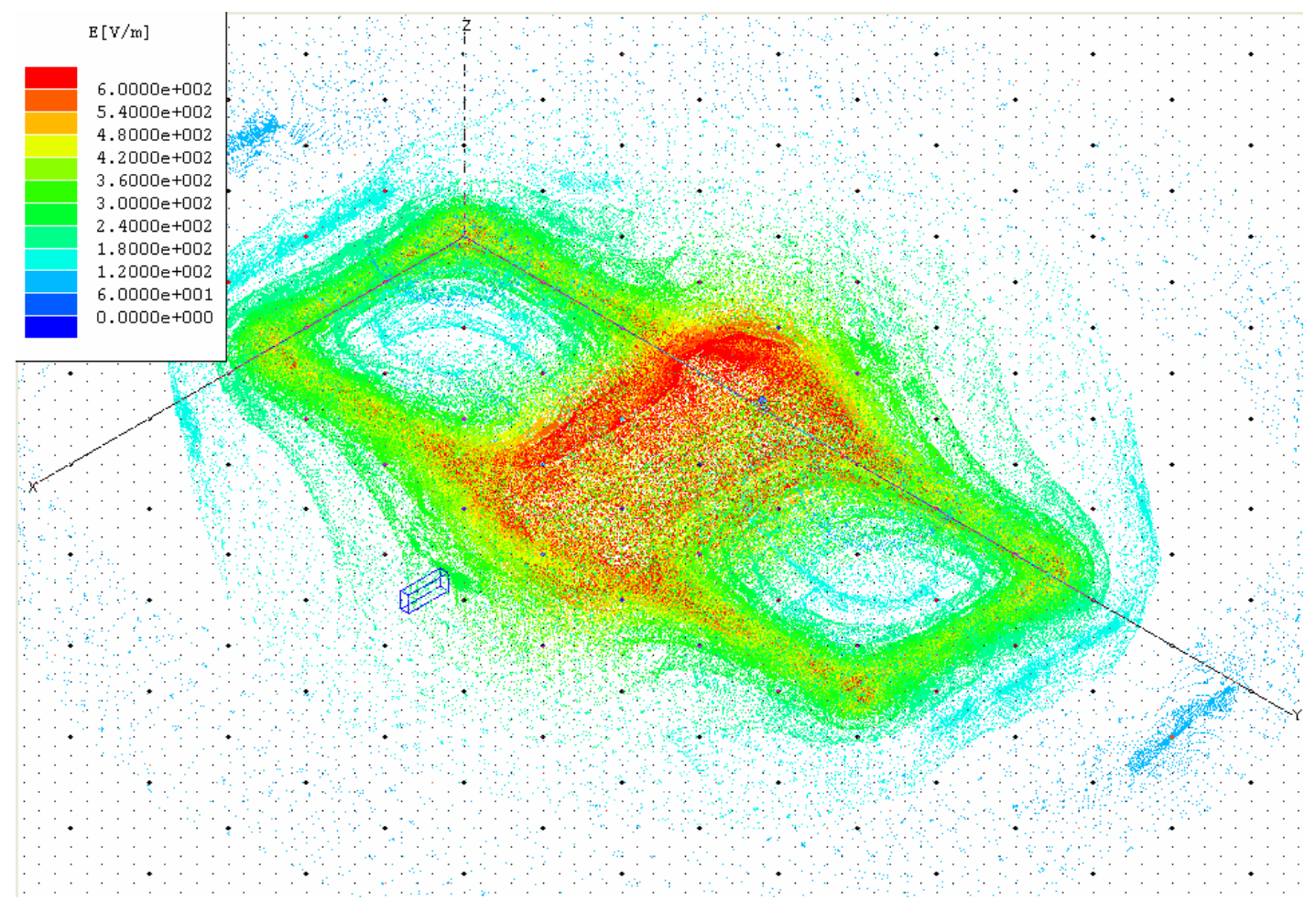

Figure 32 3-d 1 mm gap AppliedVoltage $=7 \mathrm{~V}$ 


\subsection{2-D Simulations of Gold-Chrome Microelectrode Structures}

The Simulations of the Gold-Chrome electrode structures took into account the thickness of the gold chrome structures which were $400 \mathrm{~nm}$. The collodion membrane has a thickness of 200nm; the PECVD silicon dioxide has a thickness of 1 micron and also the buffer solution having a conductivity of $5 \mathrm{mS} / \mathrm{cm}$. The size of electrodes was kept constant at 5 microns in the simulations while the distance between the electrodes was varied as 10, 20 and 40 microns. As mentioned earlier the voltages in the simulations were determined keeping in view the reported response [1] of the actin myosin system. Field strengths of more than $5000 \mathrm{~V} / \mathrm{m}$ would cause the actomyosin system to malfunction.

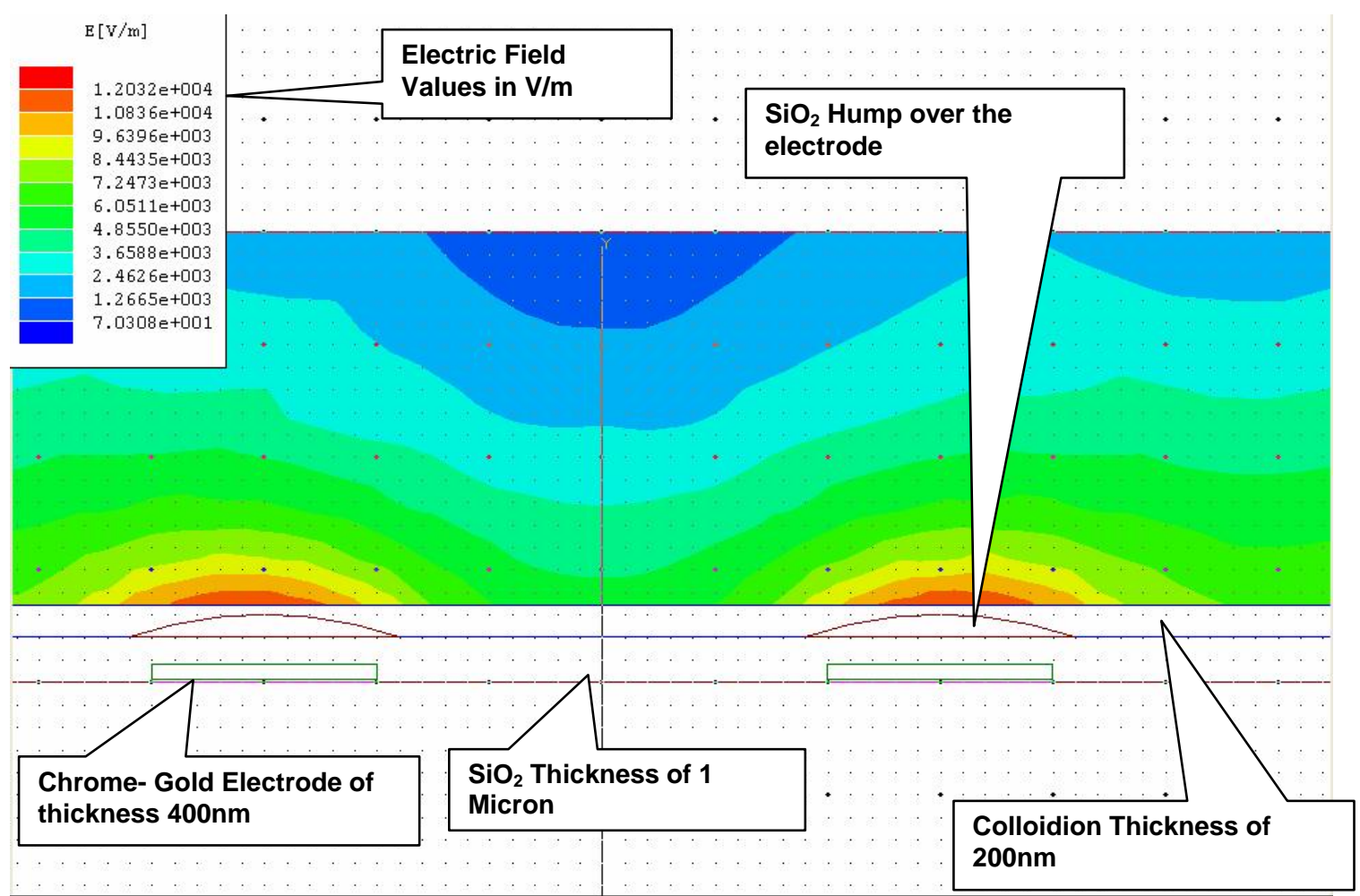

Figure 33 Index of the simulated microelectrode structures

\subsubsection{Simulations of microelectrodes with gap 10 microns}




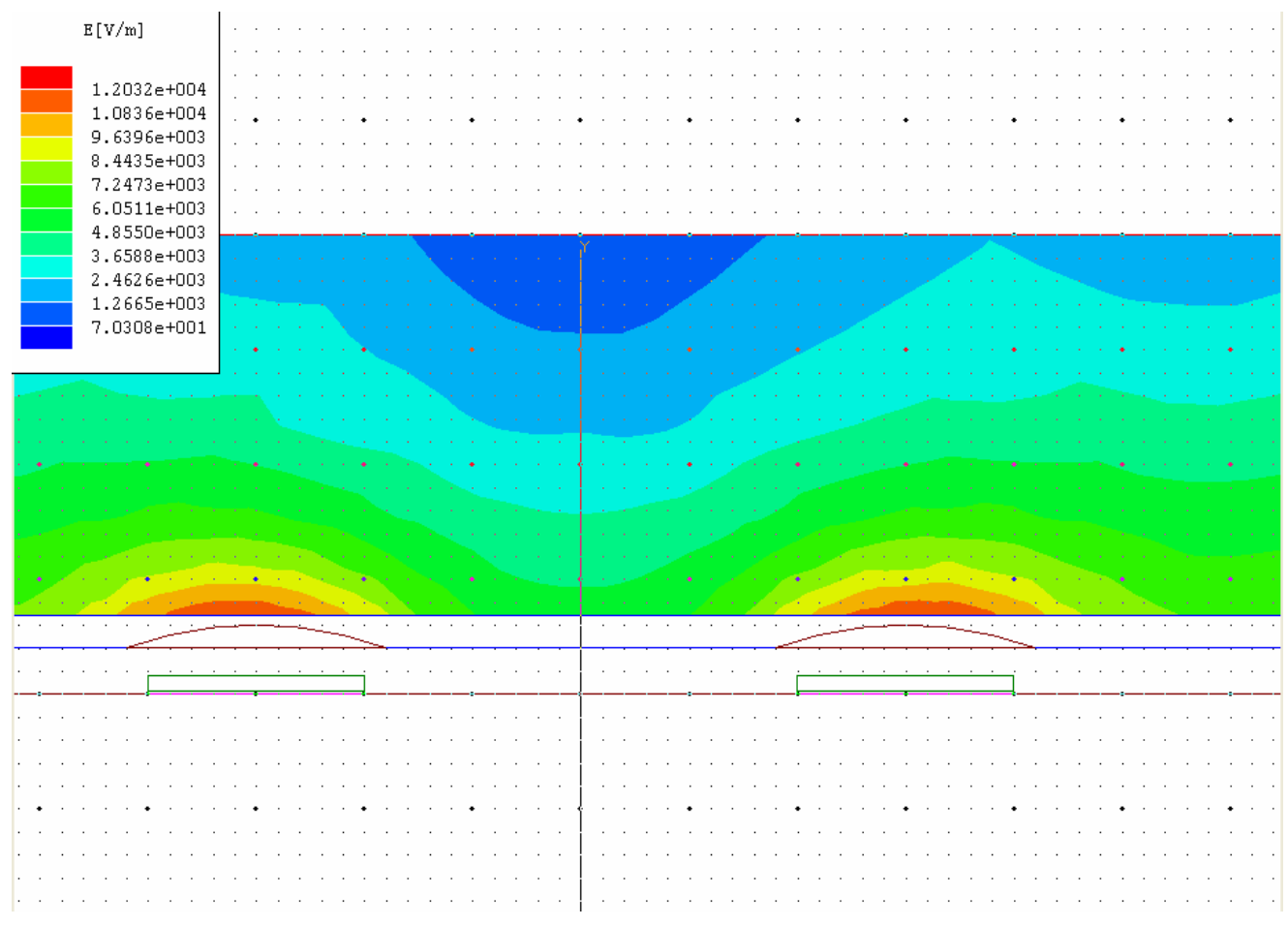

Figure $3410 \mu \mathrm{m}$ gap Applied Voltage $=0.5 \mathrm{~V}$

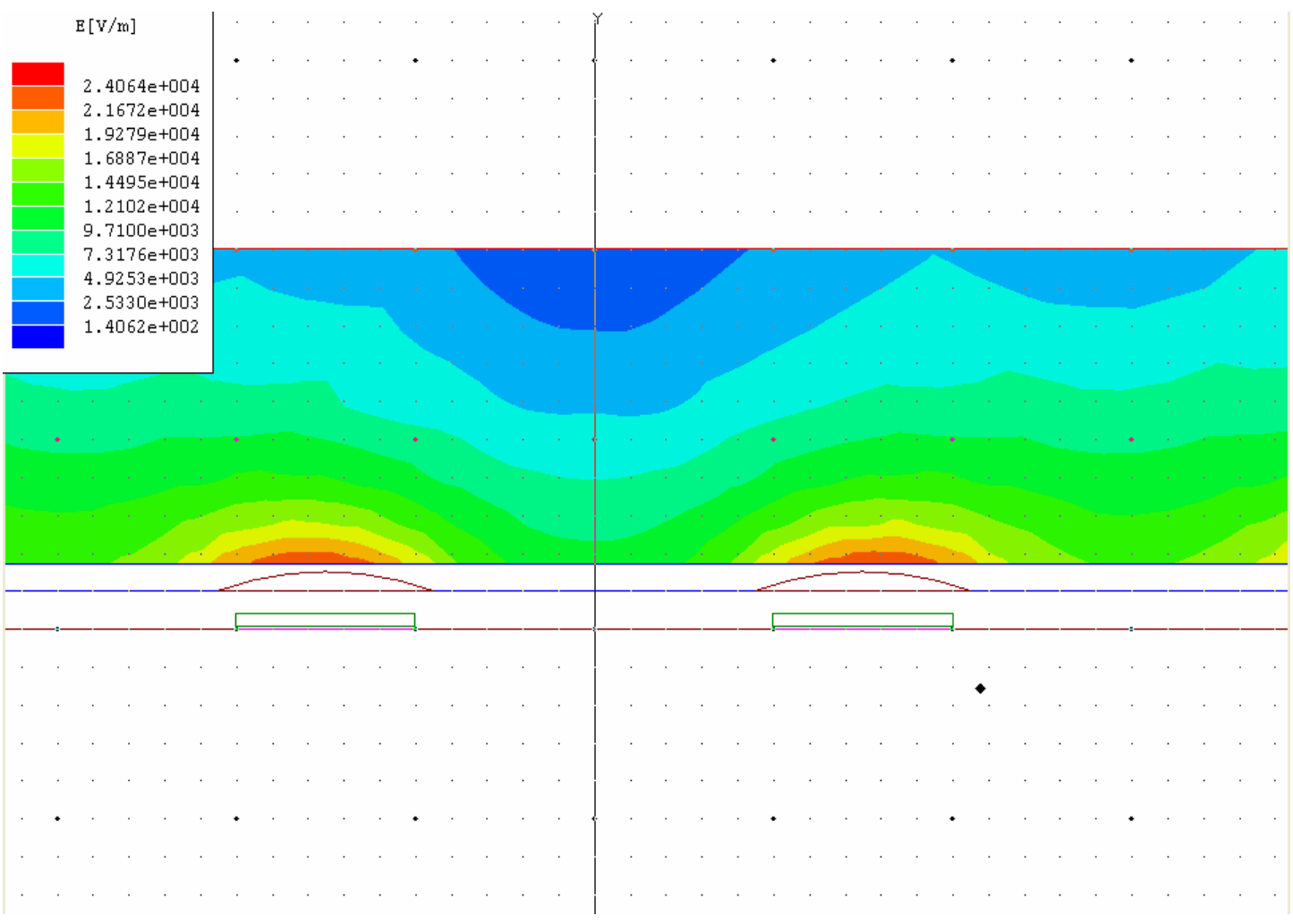

Figure $3510 \mu \mathrm{m}$ gap Applied Voltage $=1 \mathrm{~V}$ 


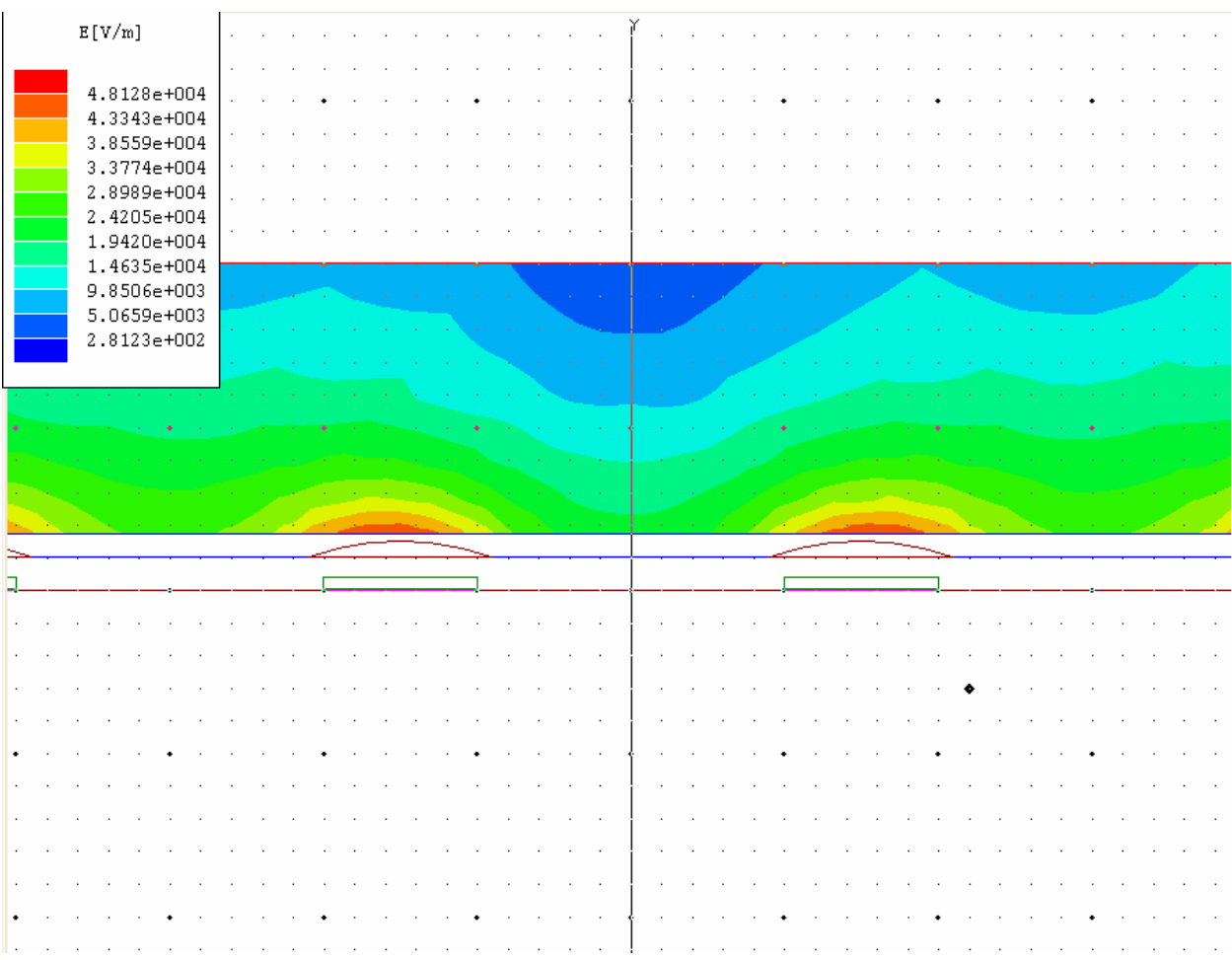

Figure $3610 \mu \mathrm{m}$ gap Applied Voltage $=2 \mathrm{~V}$

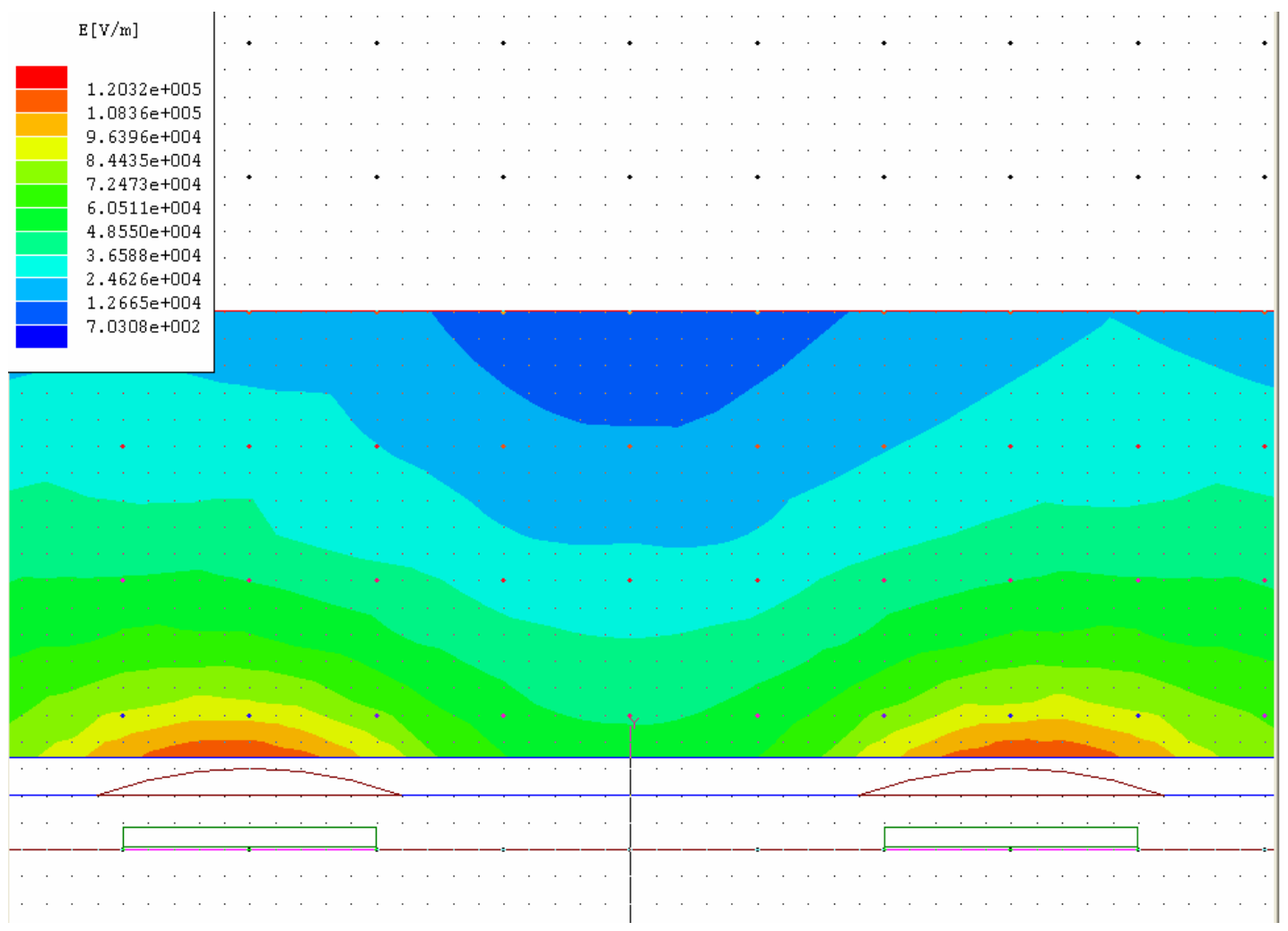

Figure $3710 \mu \mathrm{m}$ gap Applied Voltage $=\mathbf{5 V}$ 


\subsubsection{Simulations of microelectrode with gap of 20 microns}

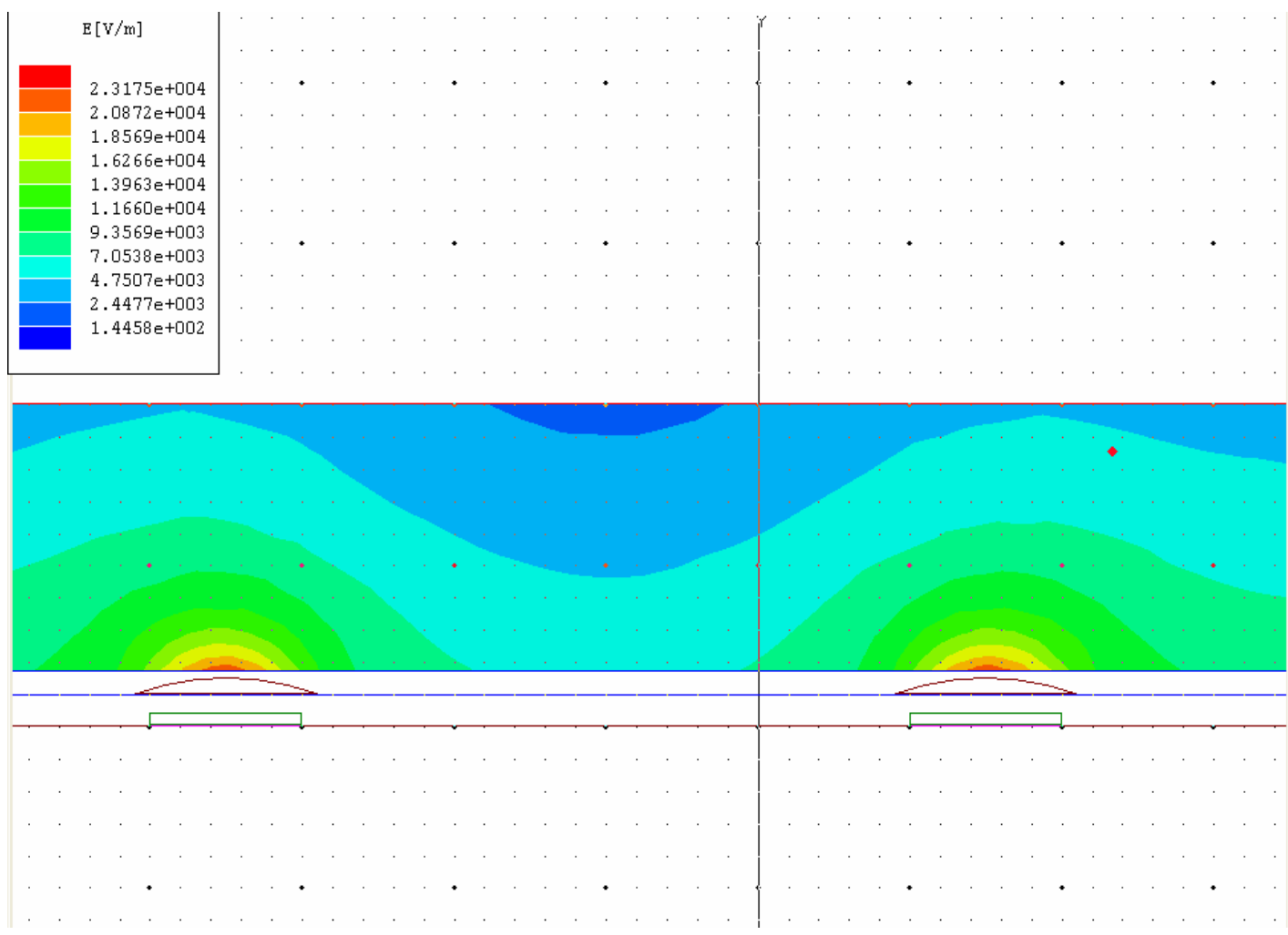

Figure $3820 \mu \mathrm{m}$ gap Applied Voltage $=0.5 \mathrm{~V}$

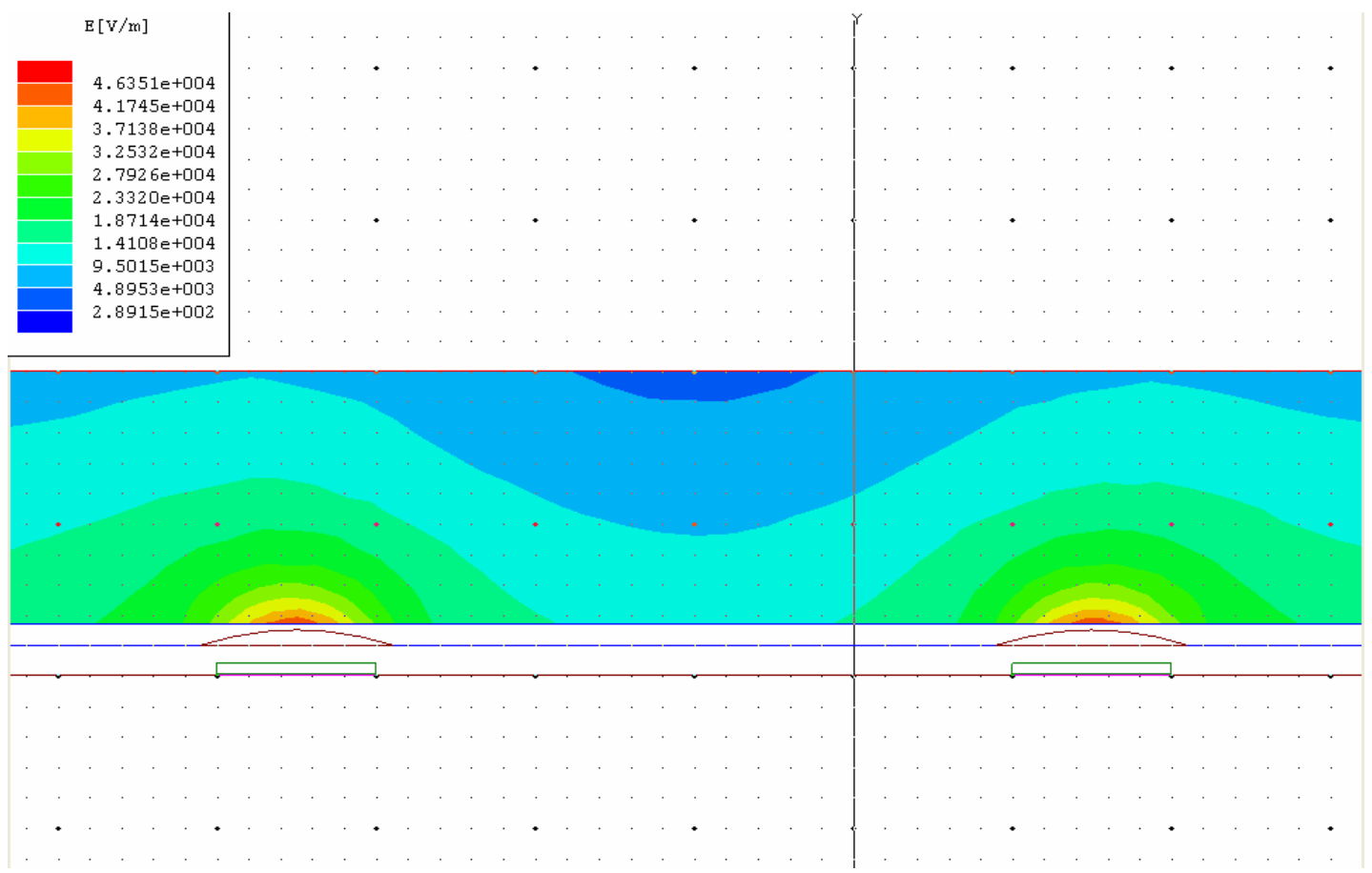

Figure 39 20 $\mu \mathrm{m}$ gap Applied Voltage $=1 \mathrm{~V}$ 


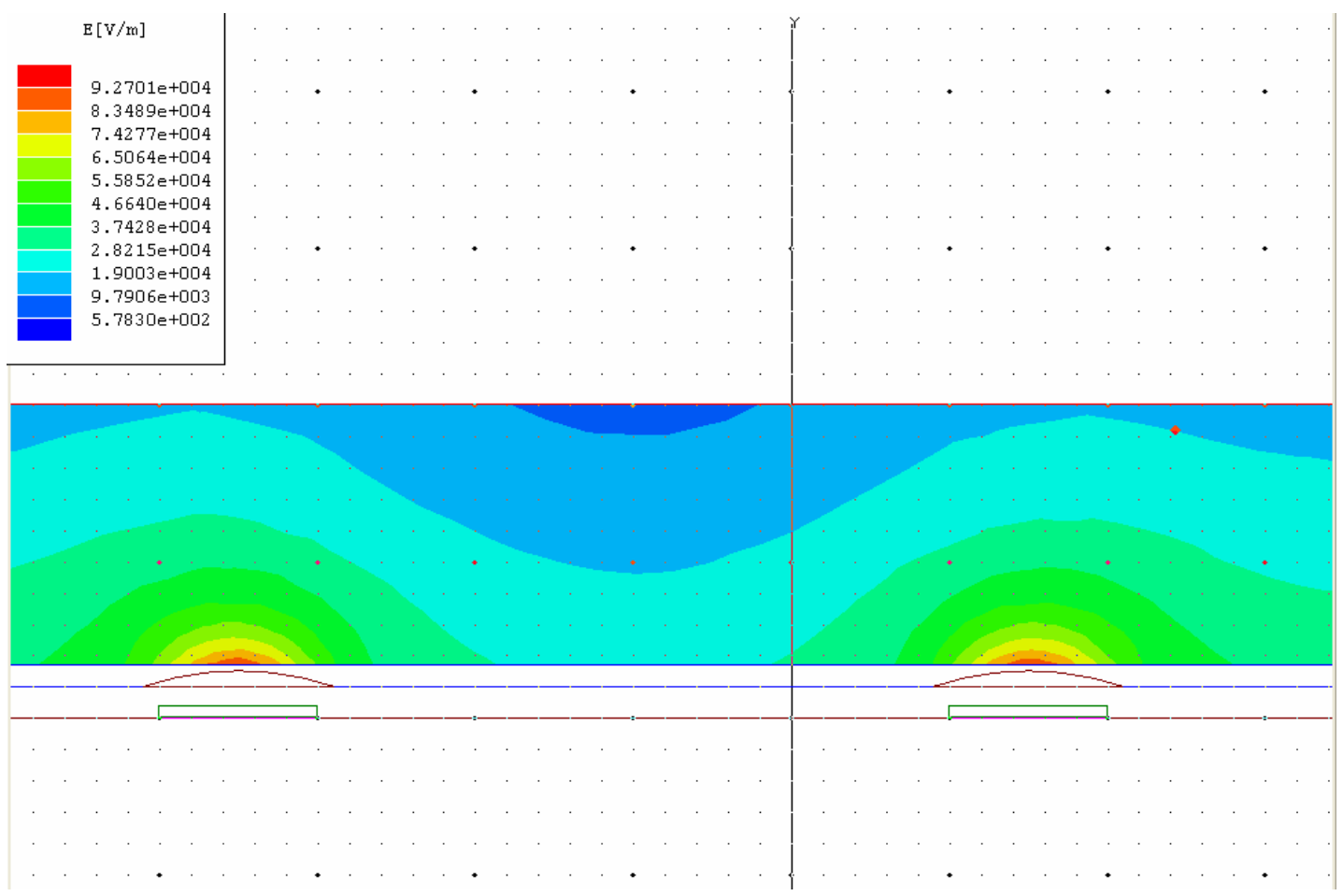

Figure $4020 \mu \mathrm{m}$ gap Applied Voltage $=2 \mathrm{~V}$.

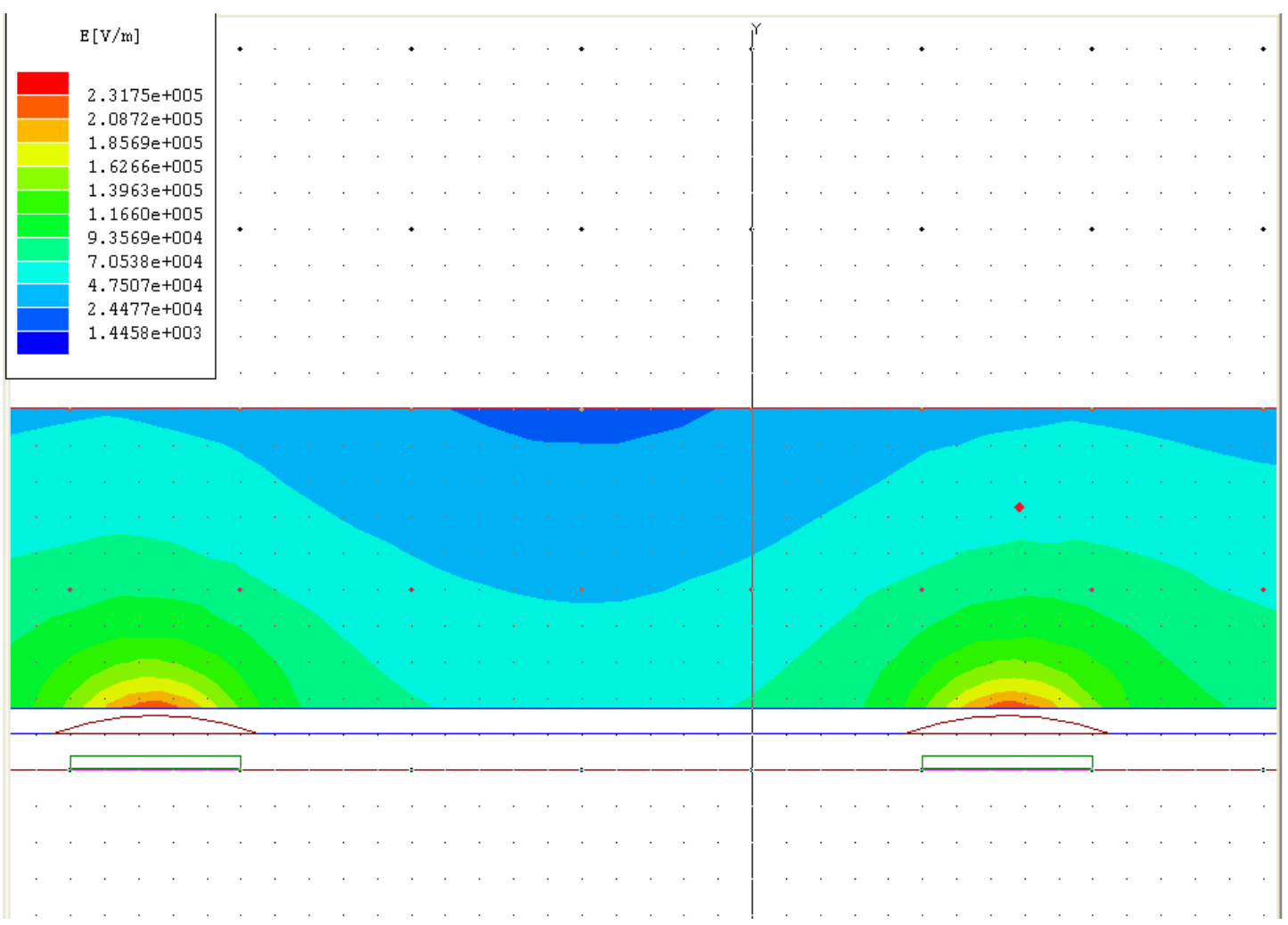

Figure $4120 \mu \mathrm{m}$ gap Applied Voltage $=5 \mathrm{~V}$ 


\subsubsection{Simulation of microelectrodes with gap of $\mathbf{4 0}$ microns}

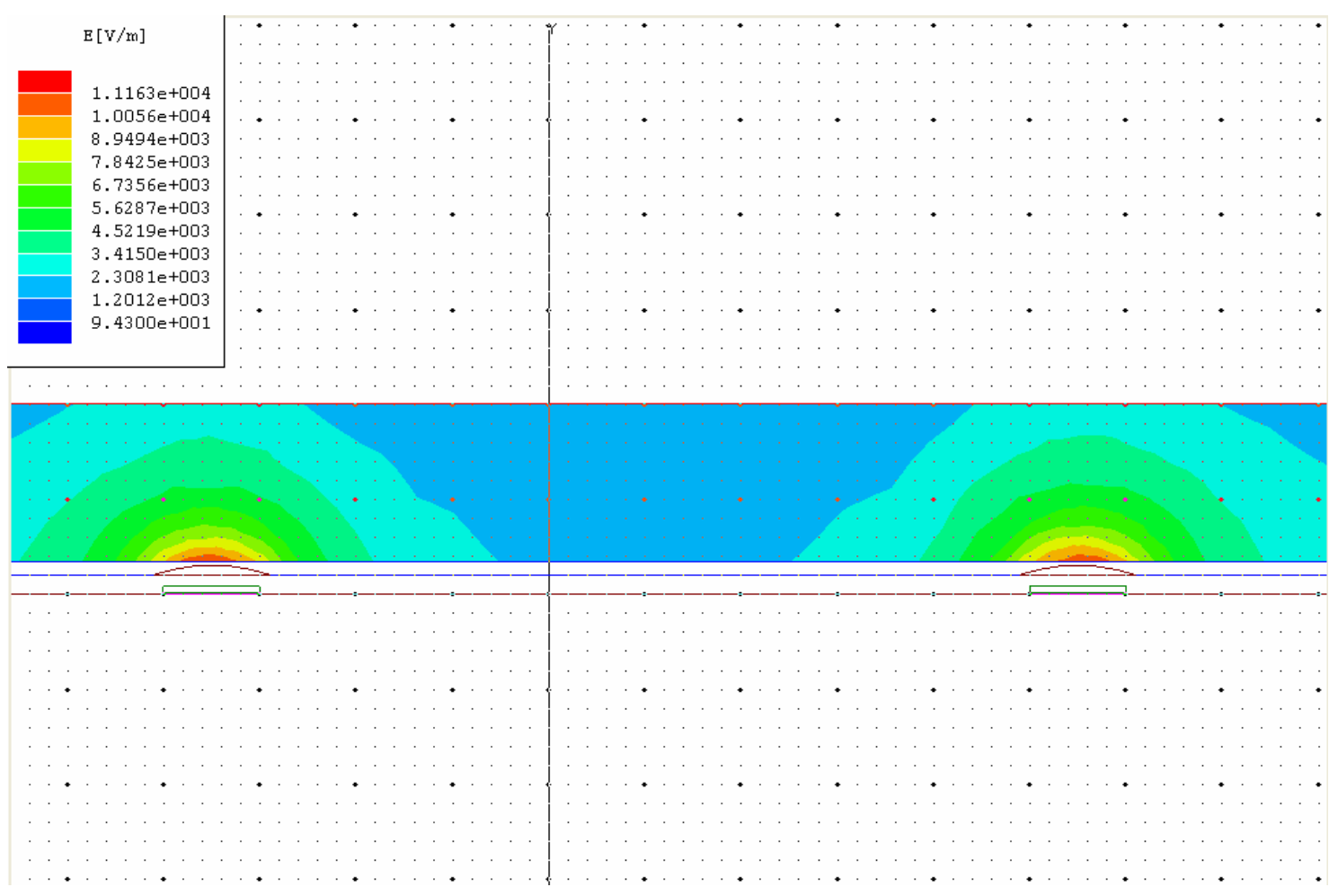

Figure $4240 \mu \mathrm{m}$ gap Applied Voltage $=0.5 \mathrm{~V}$

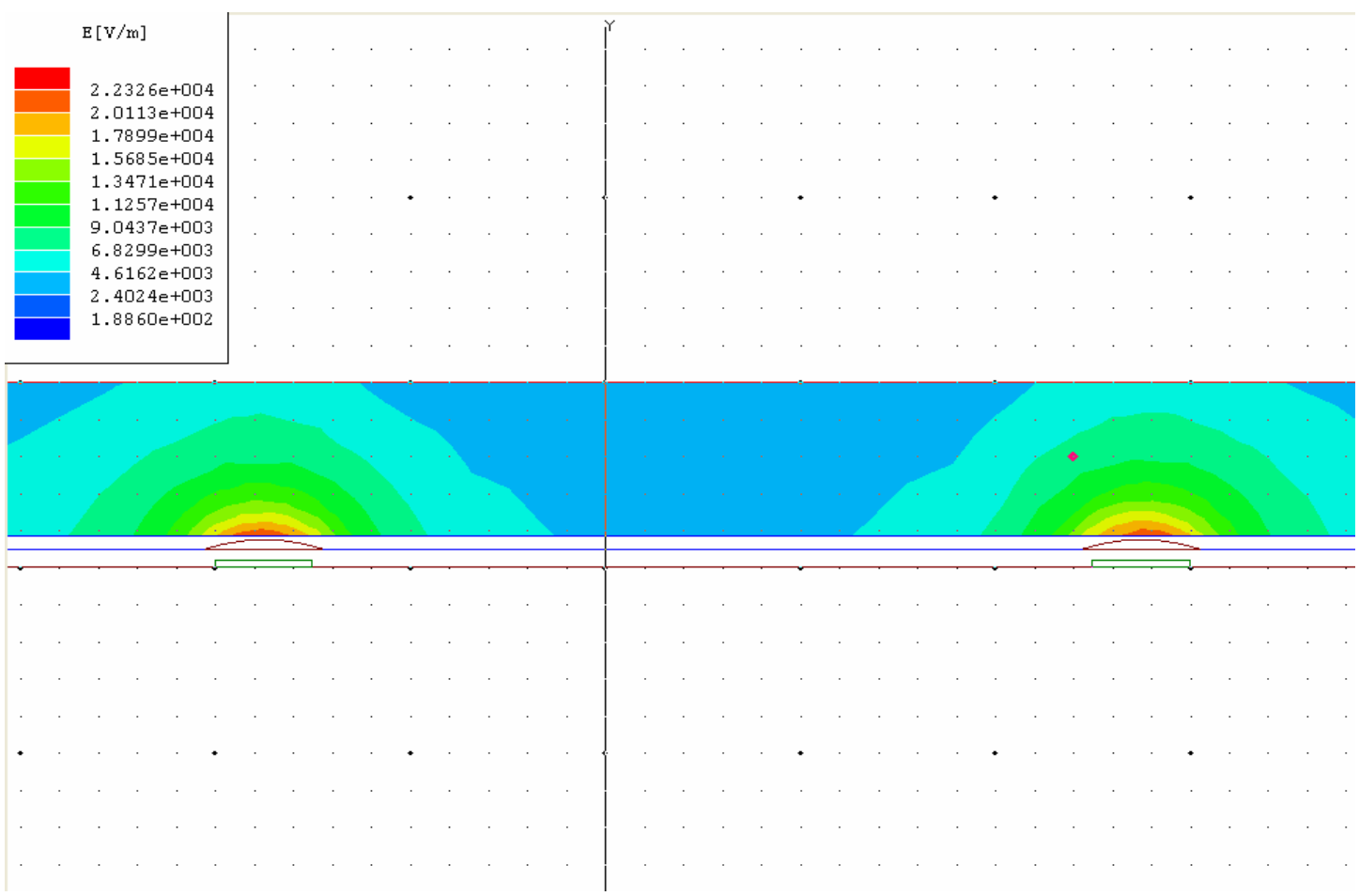

Figure $4340 \mu \mathrm{m}$ gap Applied Voltage $=1 \mathrm{~V}$ 


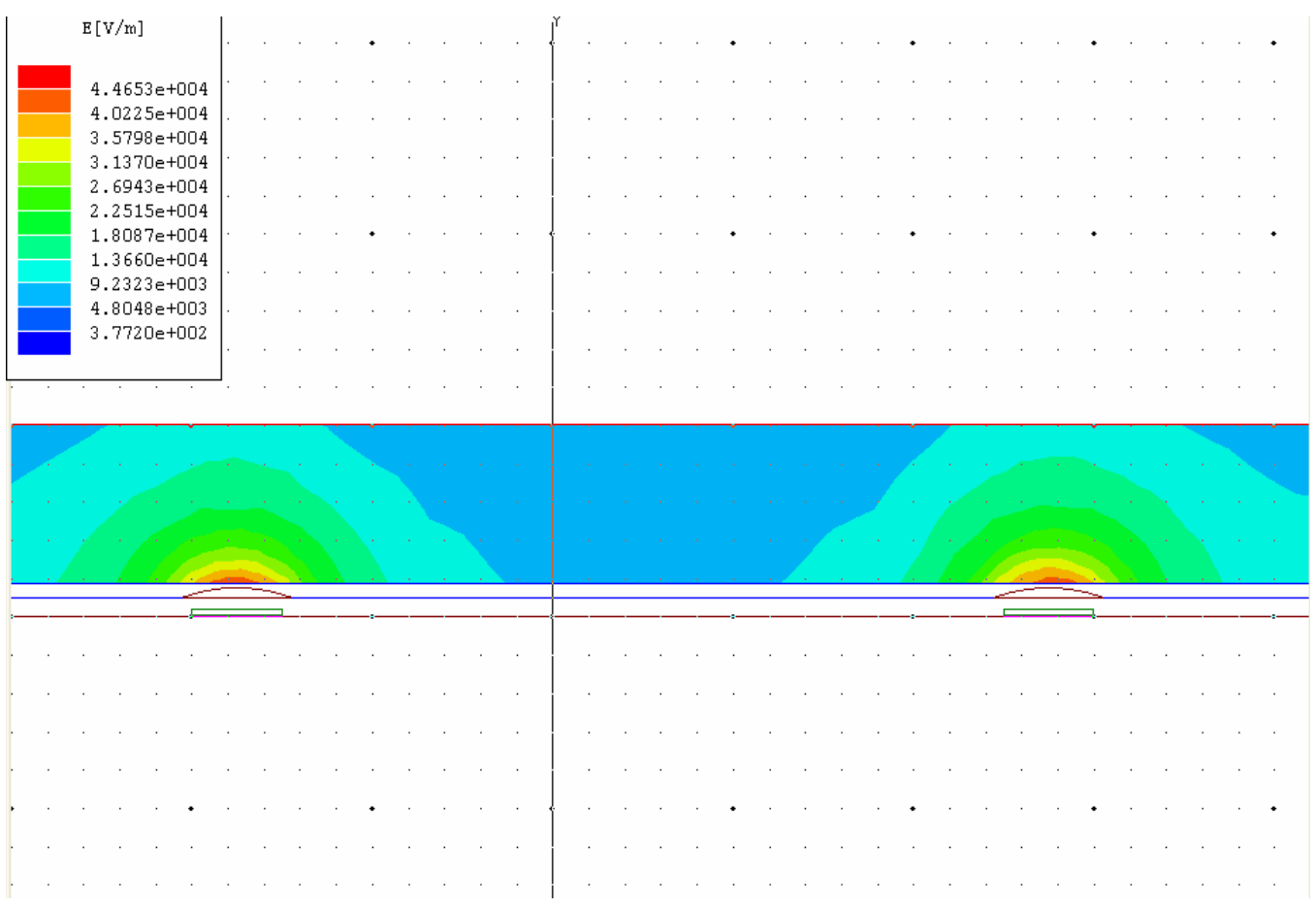

Figure 44 40 $\mu \mathrm{m}$ gap Applied Voltage $=2 \mathrm{~V}$

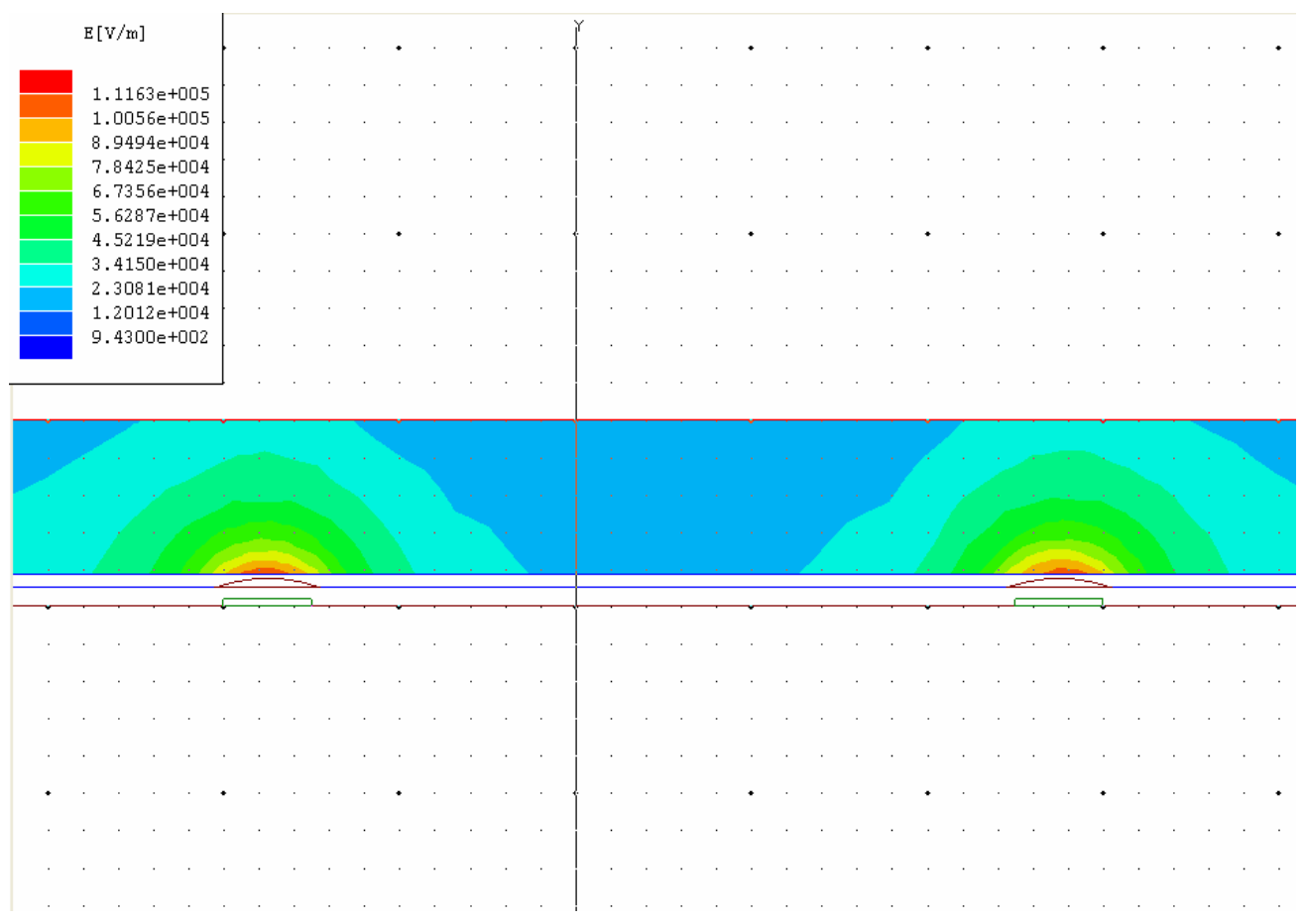

Figure $4540 \mu \mathrm{m}$ gap Applied Voltage $=5 \mathrm{~V}$

The next chapter describes the mask design and the fabrication methods employed to fabricate the chip. 


\section{Chapter 4. Design and Fabrication}

An electrode structure was designed for the purpose of observing the motility assay of the Actin-Myosin protein system in vitro. The intention was to observe the movement of the molecular motor system under the influence of applied DC electric fields. This chapter describes in detail the design of the masks used in this project and also the protocols and process flow of fabrication of the electrode chip. The macro electrodes were made out of Chrome; while the microelectrodes were made of Gold-Chrome. To understand at a more rudimentary level the behavior of Actin filament mobility in the buffer solution, a bank of chrome electrodes were also created. These had a very basic design which would be further explained in the chapter

\subsection{Gold Chrome Microelectrode Mask Design}

The mask design of the electrode structure was done using $\boldsymbol{L}$-Edit a CAD tool for mask designing. Electric field simulation of the microelectronic motility assay was undertaken to determine the proper electrode shape, size, spacing, and voltage needed to affect actin alignment without causing degradation of motor proteins in high e-fields. The structure of the mask pattern consisted of a series of interdigitated electrode sets with different spacing and different sizes of electrodes. The electrode design also consisted of a set of circular electrodes with different angles of orientation. The pattern of the electrode chip, shown in Figure, has three sets of parallel electrode patterns and four circular electrode structures of varying dimensions and angles. 


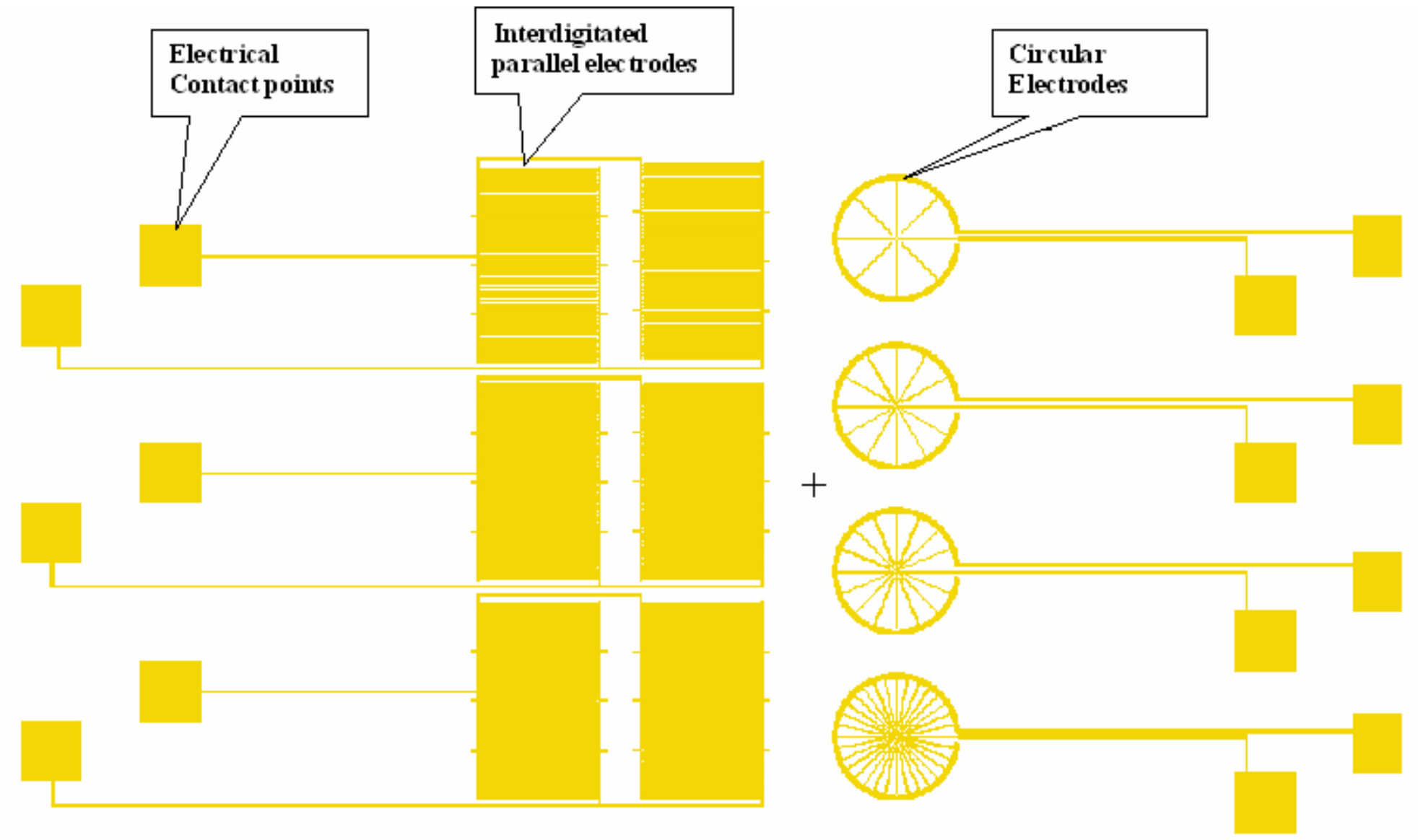

Figure 46 Structure of the mask. 
The parallel electrode patterns have varying sizes of the electrodes and the widths between them. Each parallel electrode pattern has varying size of electrodes keeping the width between the electrodes constant. i.e. the first pattern has electrode widths varying from $5-10-15-20 \mu \mathrm{m}$ keeping the width between the electrodes as $10 \mu \mathrm{m}$. The second pattern has the same electrode width variation keeping the space width constant at $20 \mu \mathrm{m}$. Similarly the third pattern has a space width of $40 \mu \mathrm{m}$. The circular electrodes have widths of $20 \mu \mathrm{m}$. The circular patterns have an angular variation of electrodes. The angles of variation are $11.25^{\circ}, 22.5^{0}, 30^{0}, 45^{0}$.

The pattern was imprinted on a Chrome Quartz mask. The critical dimension of the mask was $5 \mu \mathrm{m}$ and the tolerance was $0.5 \mu \mathrm{m}$. The Chrome Quartz masks facilitate a transmission of more than $90 \%$ of UV light during photolithography process.

\subsection{Chrome Macro Electrode Mask design}

The chrome electrode structure was utilized for characterizing and determining the mobility of Actin in the buffer solution. The chrome electrode structure was fabricated by using a set of black cardboard pieces which were scaled to the dimensions of a microscope slide. The cardboard pieces were scaled to give gaps of $1 \mathrm{~mm}, 2 \mathrm{~mm}, 5 \mathrm{~mm}$ and $10 \mathrm{~mm}$ between the chrome. The mask can be diagrammatically represented as shown below. 


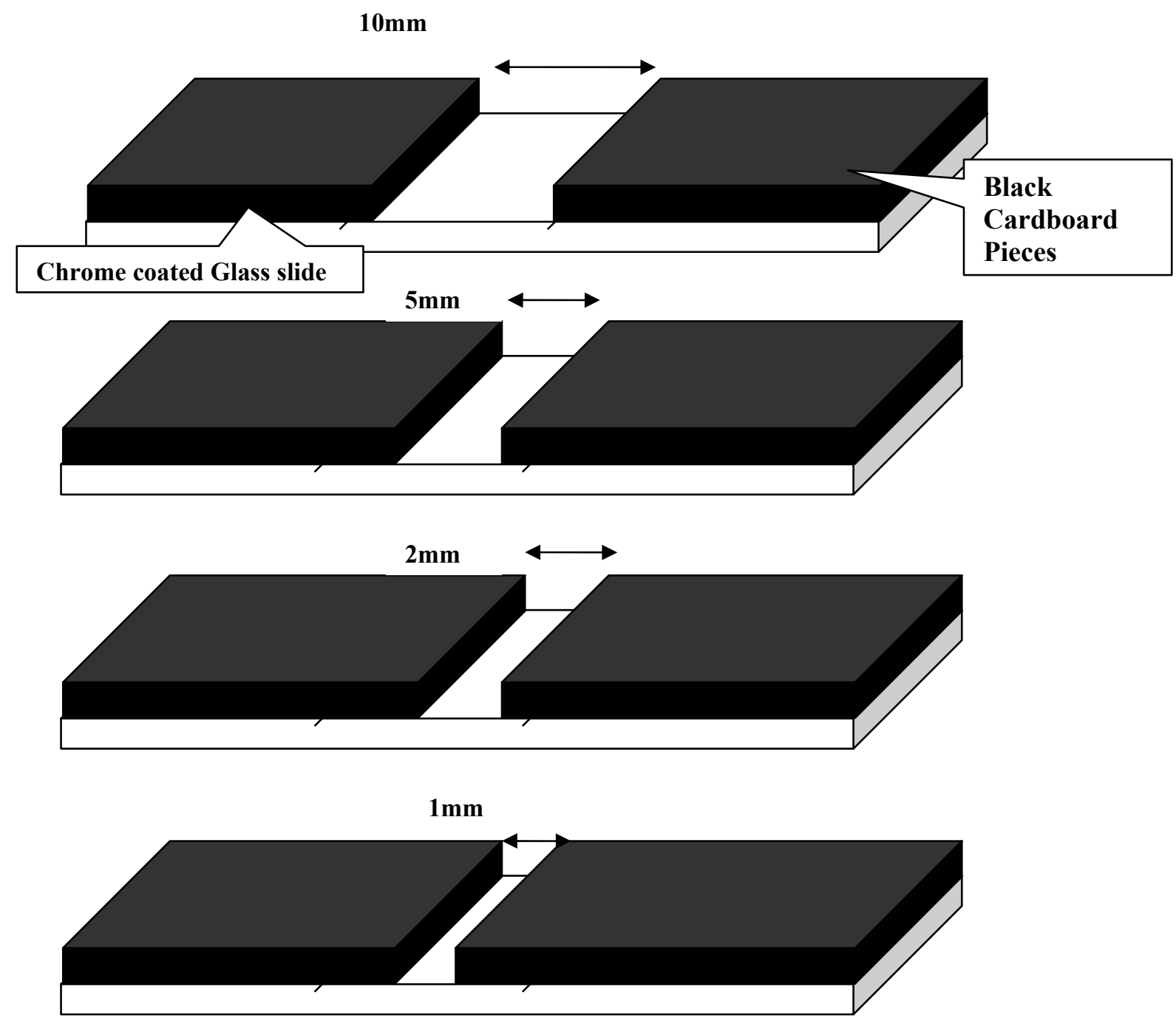

Figure 47 Macro electrode masks

The black card board pieces are approximately $0.5 \mathrm{~mm}$ thick. These cardboard pieces act as a mask. They are required to be in black color and opaque so that they don't allow the UV rays from the aligner to pass through them. 


\subsection{Fabrication of Micro and Macro Electrode structures}

The fabrication process of electrodes, both, Gold-Chrome and Chrome involves these thin film deposition and patterning processes in chronological order.

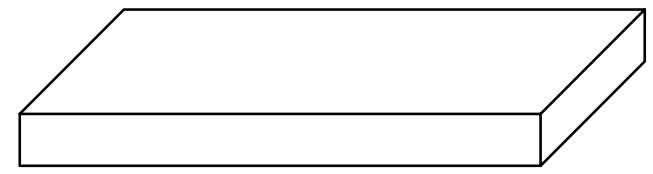

A

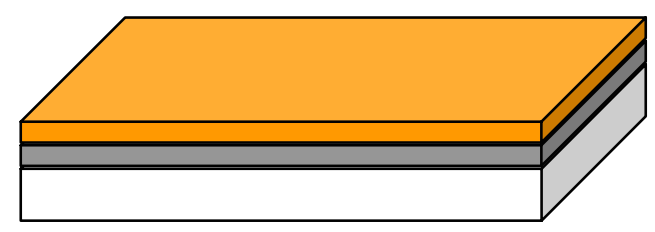

C

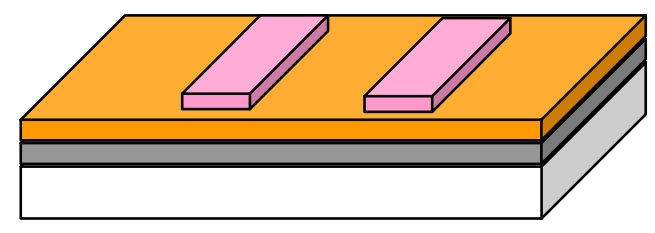

$\mathbf{E}$

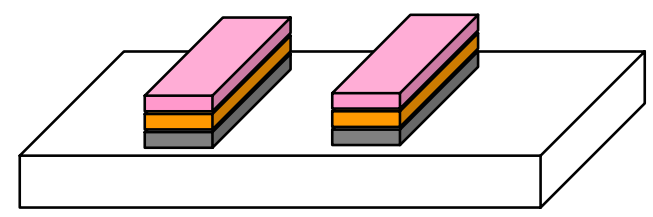

G

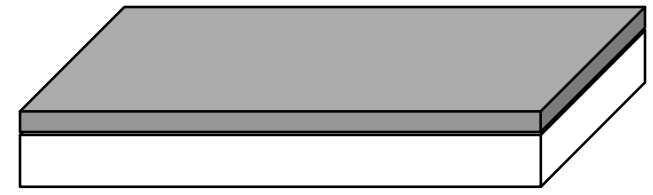

B

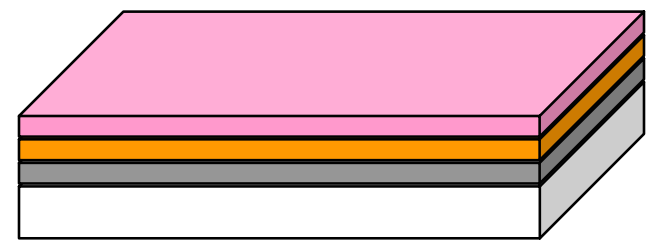

D

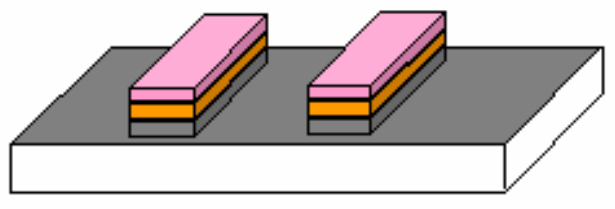

$\mathbf{F}$

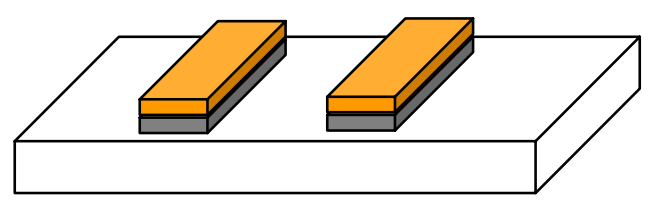

H

Figure 48 Graphical representation of the process flow of the microelectrode fabrication 


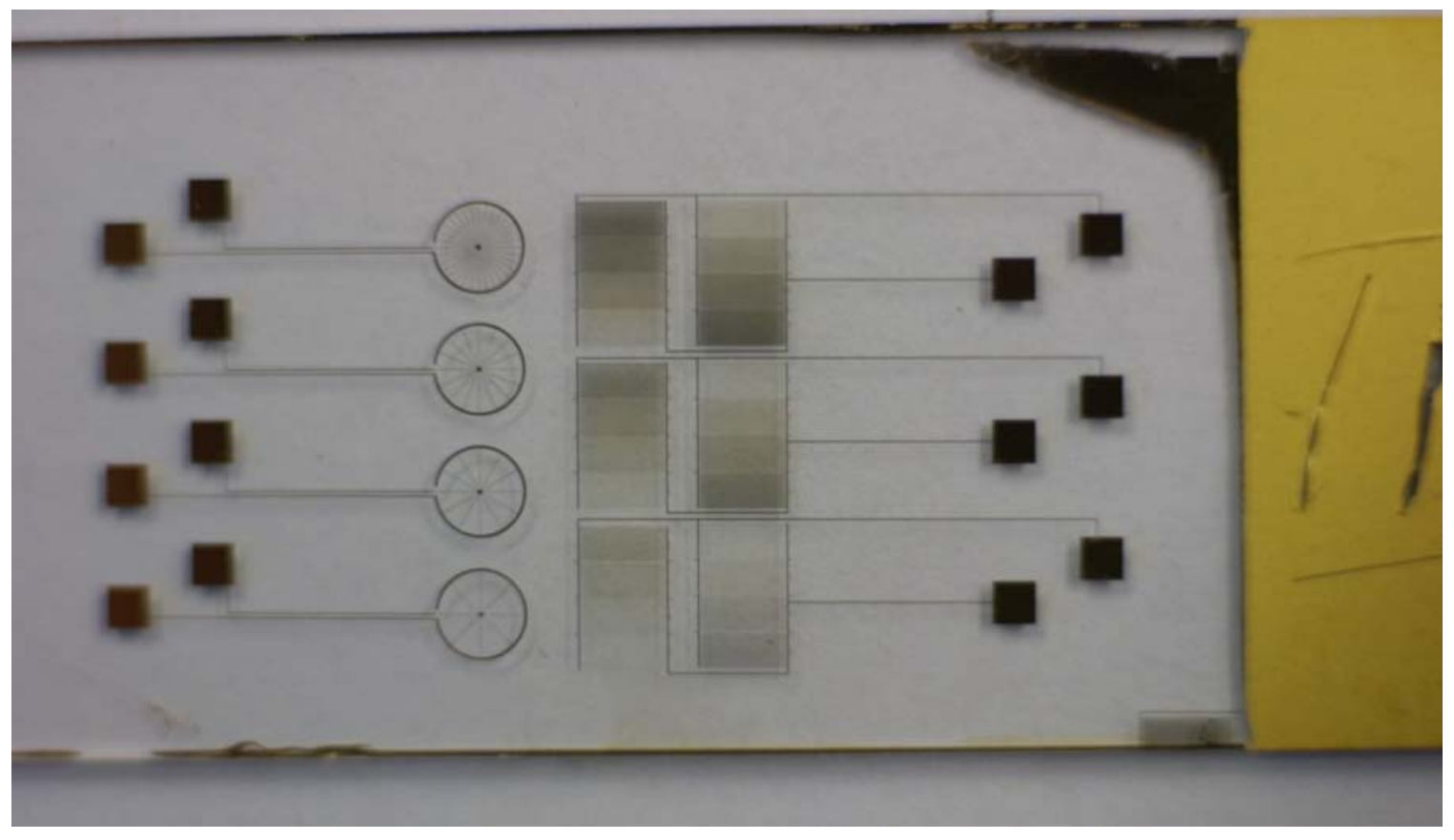

Figure 49 Electrode structures on glass slide after fabrication

\subsubsection{Fabrication notes}

a) The process beings with cleaning of the glass slide with the piranha bath process

b) An electron beam evaporator (Temescal BJD-2000) was employed in the deposition of chrome (for the microelectrode, gold-chrome process). The process table at the end of the chapter lists the time and the electrical current used in the deposition for the thickness of the metal deposited. In the fabrication of macro electrode the sputtering station (CVC 610) was employed for the deposition of chrome, the details of the parameters are listed in the process table at the end of the chapter.

c) In case of a microelectrode, the deposition of gold was done in the e-beam evaporator without breaking the vacuum, after the deposition of chrome. 
d) A layer of positive photoresist was spin applied, soft baked. The details are listed in the process table.

e) The substrate is then subjected to a photolithography process with UV rays from Karl-Suss MA-6 aligner (320nm wavelength) using a photomask of the layout in case of the microelectrodes, in the macro chrome electrode case the mask was black cardboard pieces structured to the required dimensions. The resulting layout pattern is formed in the photoresist during the development process, the photoresist in areas exposed to UV rays are removed. The process flow figure represents the outlines. The process table lists the details of the photolithography and development process.

f) The substrate is then wet etched for gold by immersing it in GE-8111 Transene. The etch times are listed in the process table. The substrate is rinsed in DI water after the gold etch.

g) The substrate is consequently wet etched to remove the chrome using a chrome mask etchant, after etching the substrate is dipped in $5 \% \mathrm{H}_{2} \mathrm{SO}_{4}$ and the rinsed in DI water.

h) The photoresist on the top of the structures was removed by immersing it in acetone and then subsequently in methanol. Chrome and gold structures in the form of the layout pattern are now present on the glass substrate.

i) The substrate was then subjected to a process of PECVD for deposition of $\mathrm{SiO}_{2}$. The PECVD acted as an insulating layer. 


\subsubsection{Bonding of wires}

The electrical contacts to the microelectrode slides were done with silver epoxy paste. The silver epoxy paste is a conductive paste. The copper wires used for the wiring were dipped into silver epoxy paste, and then stuck onto the contact pads of the slide. The silver epoxy was of the type A. After sticking the wires onto the slide the slide was cured in an oven at a temperature of $150^{\circ} \mathrm{C}$ for $1 / 2$ hour and at $200^{\circ} \mathrm{C}$ for 1 hour. Alternatively the curing could be carried out at a temperature of $150^{\circ} \mathrm{C}$ for $2 \mathrm{hrs}$. The curing caused the epoxy bond to solidify. After curing the wires were taped to the end of the slide to prevent the bond from coming loose because of the strain developed in the wires when handling it. The tape used was an insulation tape.

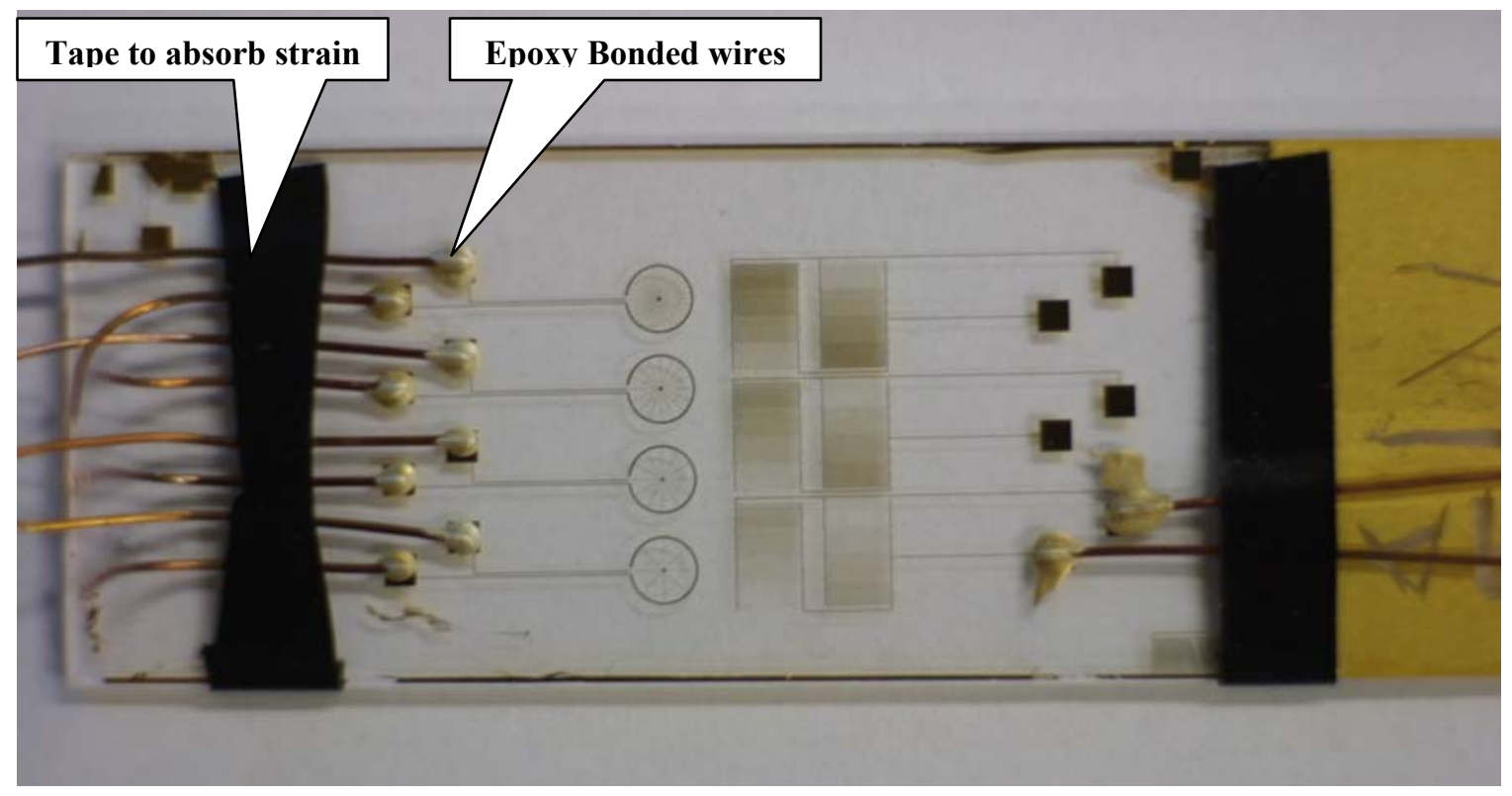

Figure 50 Electrode structure after $\mathrm{SiO}_{2}$ deposition and after bonding the wires 


\subsubsection{Fabrication of Macro electrode structures}

The fabrication of macro electrode structures involve the same steps as mentioned above. The exception is the absence of gold on the substrate. The mask for the macro electrodes is as mentioned in the section 3.2. The process parameters are listed in the process table.

\subsubsection{Bonding of wires to the Chrome slides}

Electrical wires were stuck onto the chrome slides using insulating tape. This was to allow the wire to be moving freely and also for simplicity purposes. The chrome slide with the electrical wires looks like the
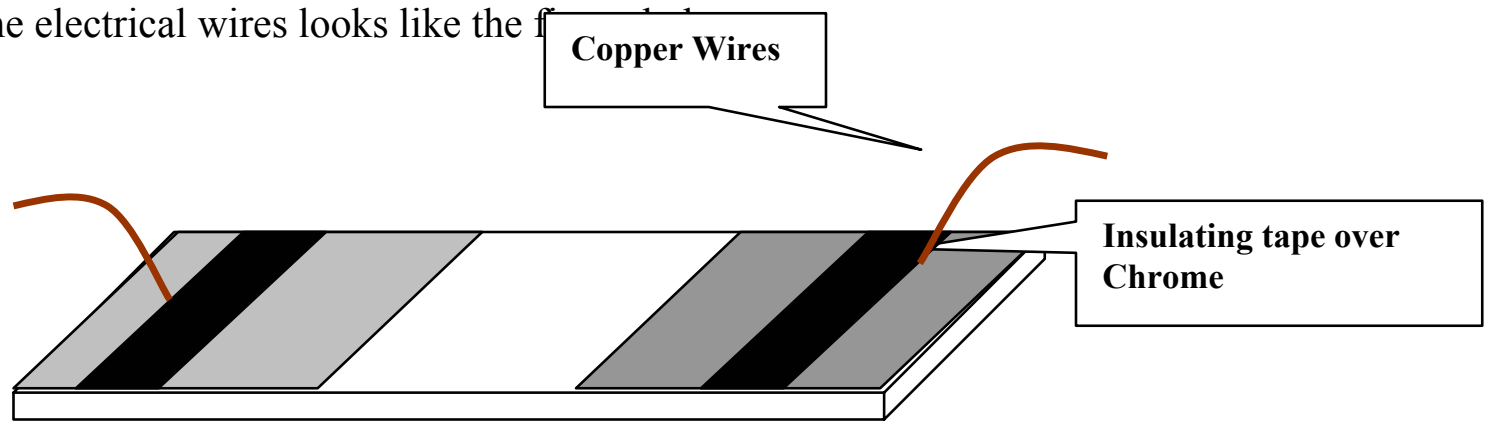

Figure 51 Graphical repsenation of bonding

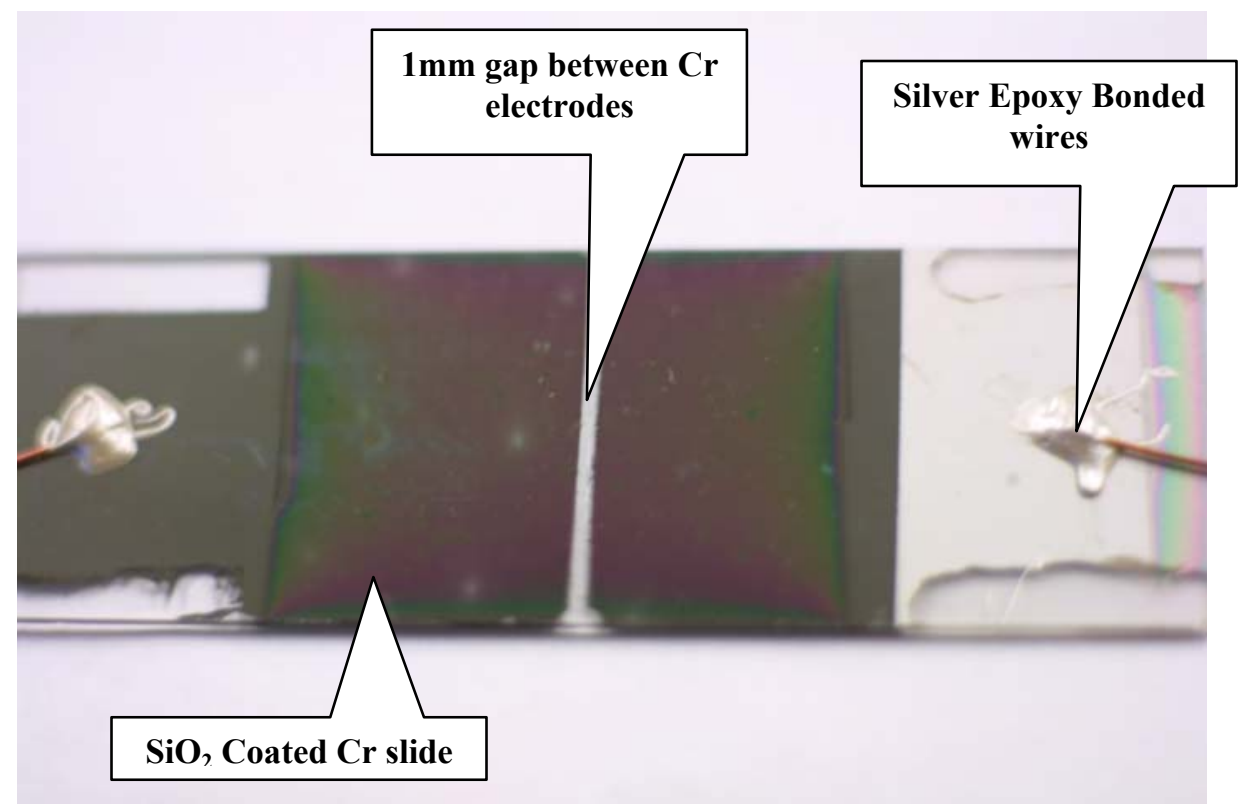

Figure 52 Macro electrode slide $1 \mathrm{~mm}$ gap with the wires bonded with epoxy 


\subsection{Process Tables}

\begin{tabular}{|c|c|c|c|c|c|}
\hline \multicolumn{6}{|c|}{ E- BEAM EVAPORTION } \\
\hline Material & \begin{tabular}{|l} 
Deposition \\
Current
\end{tabular} & $\begin{array}{l}\text { Tmeters } \\
\text { Time }\end{array}$ & \multicolumn{3}{|c|}{ Resulting thickness } \\
\hline Gold & $42 \mathrm{~mA}$ & & \multicolumn{3}{|c|}{$200 \mathrm{~nm}$} \\
\hline Chrome & $200 \mathrm{~mA}$ & & \multicolumn{3}{|c|}{$50 \mathrm{~nm}$} \\
\hline \multicolumn{6}{|c|}{ CHROME SPUTTERING PARAMETERS } \\
\hline $\begin{array}{l}\text { Vacuum } \\
\text { Pressure }\end{array}$ & \begin{tabular}{|l}
\multicolumn{1}{|c|}{ Process } \\
Process
\end{tabular} & $\begin{array}{l}\text { ameters } \\
\text { Flow }\end{array}$ & $\begin{array}{l}\text { Deposition } \\
\text { Power }\end{array}$ & $\begin{array}{c}\text { Parameters } \\
\text { Time }\end{array}$ & $\begin{array}{l}\text { Resulting } \\
\text { Thickness }\end{array}$ \\
\hline $5 \times 10^{-6}$ torr & $60 \mathrm{~m}$ Torr & $30 \mathrm{sccm}$ & $0.1 \mathrm{~kW}$ & $6 \min$ & $\approx 285 \mathrm{~nm}$ \\
\hline \multicolumn{6}{|c|}{ PHOTORESIST APPLICATION DETAILS } \\
\hline Photoresist & $\begin{array}{l}\text { Spinne } \\
\text { Speed }\end{array}$ & $\begin{array}{l}\text { Time } \\
\text { Ttings }\end{array}$ & $\begin{array}{l}\text { Photoresist } \\
\text { Thickness }\end{array}$ & $\begin{array}{l}\text { Softbake P } \\
\text { Temperature }\end{array}$ & $\begin{array}{l}\text { arameters } \\
\text { Time }\end{array}$ \\
\hline AZ 5214E & 4000rpm & $1 \mathrm{~min}$ & $\approx 1.8 \mu \mathrm{m}$ & $100^{\circ} \mathrm{C}$ & $1 \mathrm{~min}$ \\
\hline \multicolumn{6}{|c|}{ EXPOSURE AND DEVELOPMENT PARAMETERS } \\
\hline \multicolumn{3}{|c|}{ Exposure Settings } & \multicolumn{3}{|c|}{ Development parameters } \\
\hline Type & Power & Time & Developer & Concentration & Time \\
\hline Soft contact & $1.9 \mathrm{~mW} / \mathrm{cm}^{2}$ & $84 \mathrm{sec}$ & AZ $312 \mathrm{MIF}$ & $1: 4$ & $\approx 1 \min 55 \mathrm{sec}$ \\
\hline \multicolumn{6}{|c|}{ GOLD ETCHING DETAILS } \\
\hline \multirow[t]{2}{*}{ Material } & \multicolumn{5}{|c|}{ Etching and Rinsing parameters } \\
\hline & Etchant & Etch & & Rinse ti & \\
\hline
\end{tabular}




\begin{tabular}{|l|l|l|l|l|l|l|}
\hline Gold & GE 8111 & \multicolumn{2}{|c|}{$\approx 2$ min 45 sec } & \multicolumn{3}{c|}{5 minutes } \\
\hline \multicolumn{7}{|c|}{ CHROME ETCH PARAMETERS } \\
\hline Material & \multicolumn{3}{|c|}{ Etching/Rinsing Parameters } & \multicolumn{2}{|c|}{ Post Etch Cleaning } \\
& Etchant $\quad$ Etch time $\quad \mathrm{H}_{2} \mathrm{SO}_{4}$ Soak DI Rinse & Acetone $\quad$ Drying \\
\hline Chrome & $\begin{array}{l}\text { Chrome } \\
\text { Mask } \\
\text { Etchant } \\
\text { (Transene) }\end{array}$ & $\approx 45 \mathrm{sec}$ & 5 min & 5 min & 5 min & $\begin{array}{l}\text { Nitrogen } \\
\text { Flow }\end{array}$ \\
\hline & & & & & & \\
\hline
\end{tabular}

The next chapter, chapter $\mathrm{V}$ describes in brevity the experimental setup, the components used to hook up the slide with the electrical connections, the visual display units and the process in which the experiment is carried out. 


\section{Chapter 5. Experimental Setup}

\subsection{Experimental set up}

The experimental set up of this project was established in the chemistry research department laboratories. The setup consisted of these following components. The graphical representation below shows the setup of a standard motility assay.

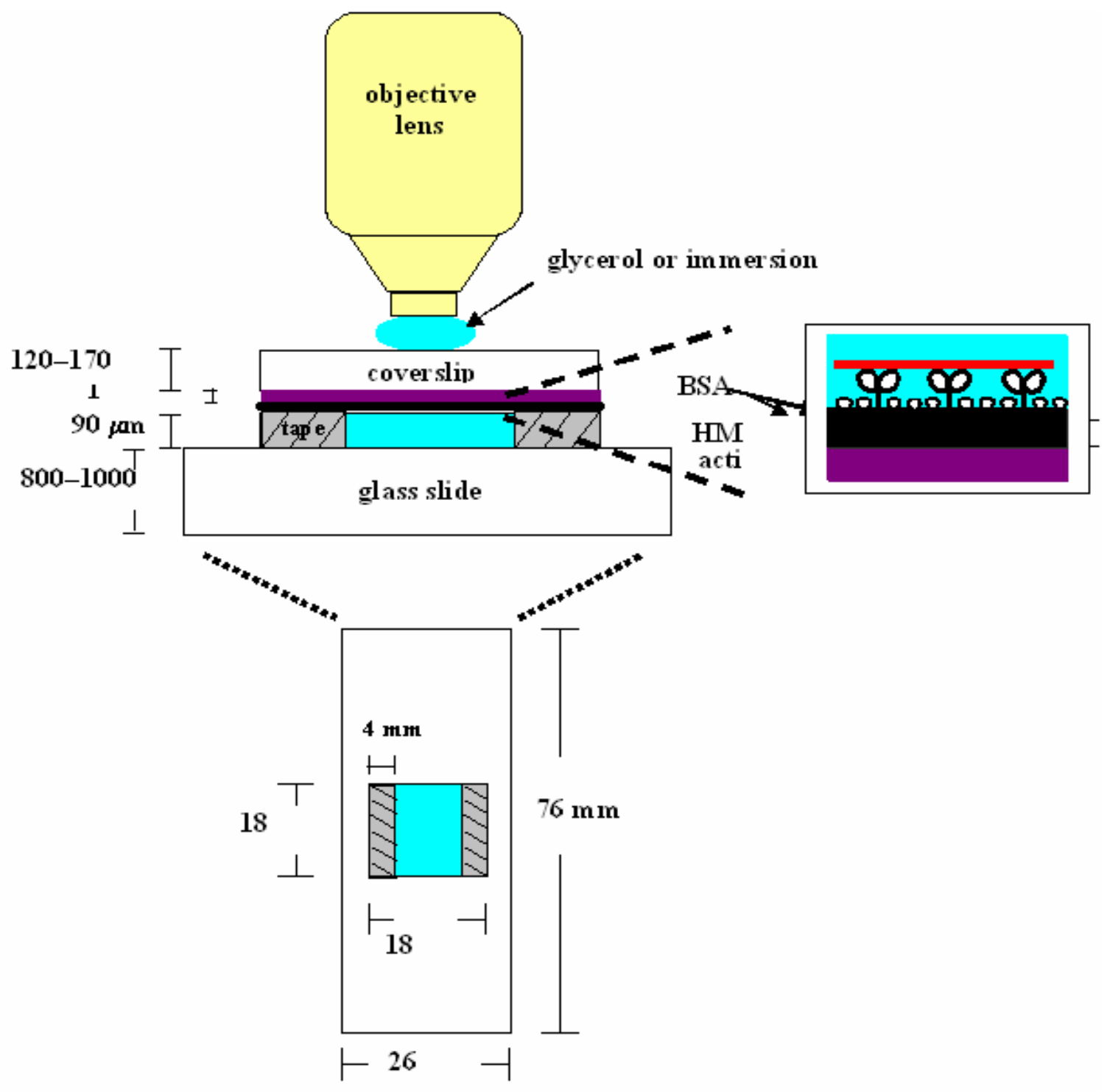

Figure 53Graphical representation depicting the setup for a standard motility assay 


\subsubsection{Microscope:}

An Olympus BH 152, upright, fluorescence microscope was used. The microscope had lens of magnification of 20X, 40X and 100X. The Working Distance (WD) of 100X lens is $0.18 \mathrm{~mm}$. The W.D of $40 \mathrm{X}$ is around $0.35 \mathrm{~mm}$. The microscope was arranged in a wooden box to provide a dark ambience while performing the experiment. Extended attachments were attached to the focusing knob and the X-Y direction movement controller knob. Holes were drilled on either sides of the box and the extendable attachments were attached. This setup provided a way to operate the microscope without opening the door of the box while performing the experiment and therefore preventing external light (room light) interfering. External light could cause interference while observing the fluorescent dyed actin filaments. The lens which were used to observe were immersion lens with either glycerol or immersion oil as the medium of observance.

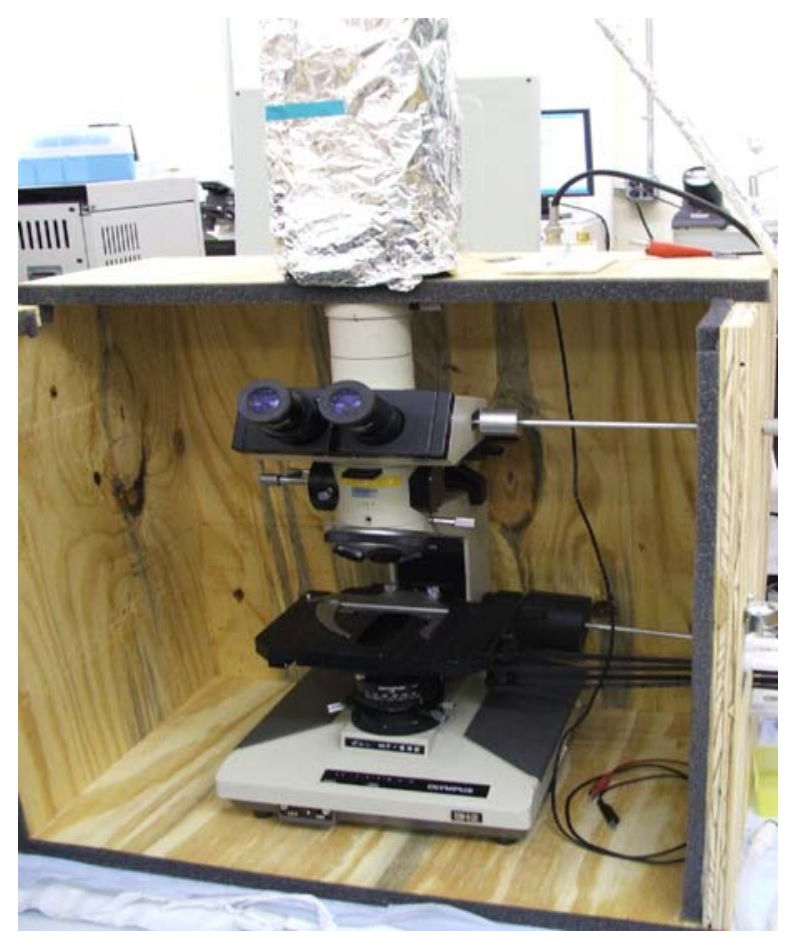

Figure 54 Microscope 


\subsubsection{Display and Recording systems:}

The visuals were seen on a large sized TV monitor (Dell). The visuals were recorded from a CCD camera (Hamamitsu). There is a timer display unit which is used to label the videos during the recording. This helps to differentiate the videos. An intensity meter was used to control the intensity of the visual from the CCD camera. A Panasonic VCR was used to record the visuals in real-time. The display system was thoroughly shielded for noise from the external sources.

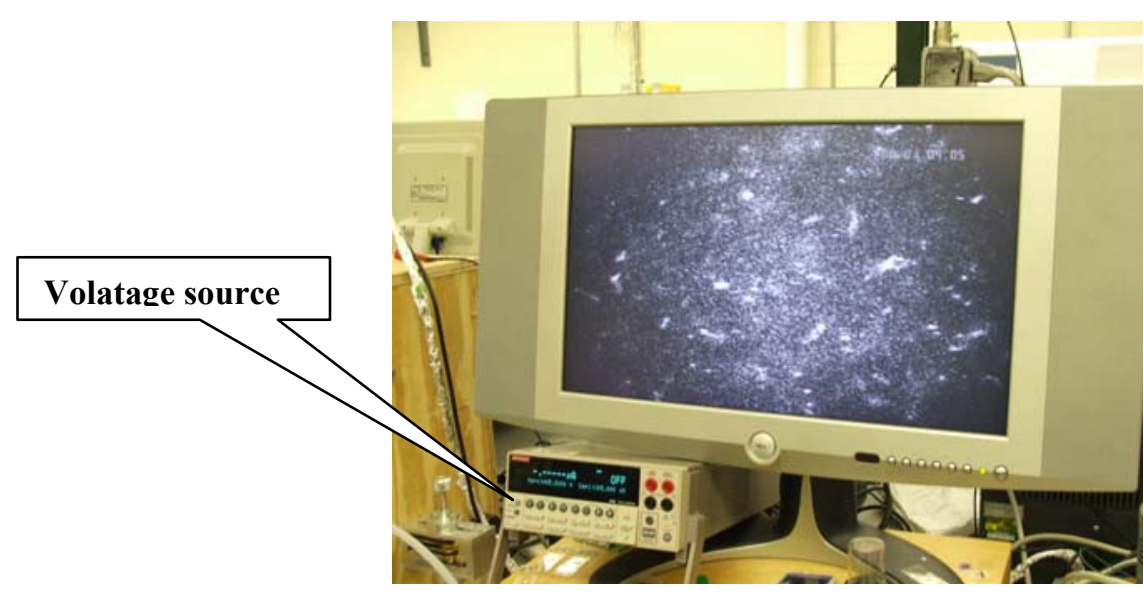

Figure 55 Flat screen Monitor used for analyzing the videos

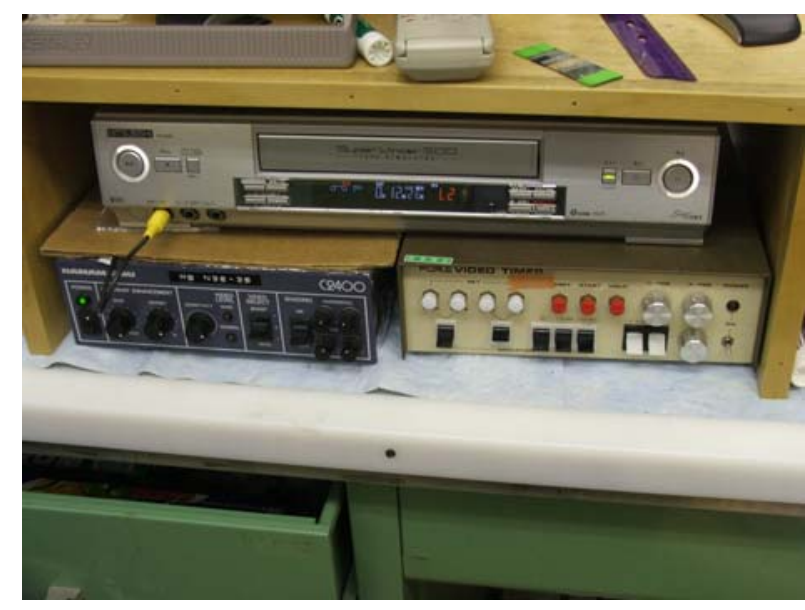

Figure 56 The video recording unit, 


\subsubsection{Motility assay solutions:}

The motility assay solution consists of the following composition of chemicals.

\section{Assay buffer}

$25 \mathrm{mM} \mathrm{KCl}(3000 \mathrm{mM} \times 1 / 120), 2 \mathrm{mM} \mathrm{MgCl}_{2}(1000 \mathrm{mM} \times 1 / 500)$,

$0.2 \mathrm{mM} \mathrm{CaCl}_{2}(200 \mathrm{mM} \times 1 / 1000), 25 \mathrm{mM}$ Imidazole $(2500 \mathrm{mM} \times 1 / 100)$ at $\mathrm{pH} 7.0$

\section{Dilution of myosin or HMM (Heavy meromyosin) into the desired concentration}

HMM in the same buffer of low ionic strength including $25 \mathrm{mM} \mathrm{KCl}$

Myosin in the different buffer of high ionic strength including $300 \mathrm{mM} \mathrm{KCl}$

\section{Bovine Serum Albumin (BSA) solution}

$1 \mathrm{mg} / \mathrm{mL}$ BSA (Fraction V) in water

\section{Dilution of F-actin labeled with rhodamine-phalloidin (RP)}

$25 \mu \mathrm{g} / \mathrm{mL}$ RP-labeled actin $(0.25 \mathrm{mg} / \mathrm{mL} \times 1 / 10)$ in assay buffer

\section{Actin solution $(100 \mu \mathrm{L})$}

Assay buffer $(94 \mu \mathrm{L})$

$10 \mathrm{mM}$ DL-Dithiothreitol (DTT, $1000 \mathrm{mM} \mathrm{1 \mu L)}$ or $14 \mathrm{mM}$ 2-Mercaptoethanol (1400 $\mathrm{mM} 1 \mu \mathrm{L})$

0 or $1.5 \mathrm{mM}$ ATP $(150 \mathrm{mM} 1 \mu \mathrm{L})$, $0.216 \mathrm{mg} / \mathrm{mL}$ Glucose oxidase $(21.6 \mathrm{mg} / \mathrm{mL} 1 \mu \mathrm{L})$

$0.036 \mathrm{mg} / \mathrm{mL}$ Catalase $(3.6 \mathrm{mg} / \mathrm{mL} 1 \mu \mathrm{L})$

$4.5 \mathrm{mg} / \mathrm{mL}$ Glucose $(450 \mathrm{mg} / \mathrm{mL} 1 \mu \mathrm{L})$

$0.25 \mu \mathrm{g} / \mathrm{mL}$ RP-labeled F-actin $(25 \mu \mathrm{g} / \mathrm{mL} 1 \mu \mathrm{L})$

\subsubsection{Nitrocellulose (Collodion)-coated coverslip}


The coverslip used for creating the flow cell was treated with the following chemicals. $0.2 \%$ Collodion $(2 \% \times 1 / 10$, Okenshoji Co. Ltd., Japan $)$ in Isoamyl acetate. $10 \mu \mathrm{L}$ of $0.2 \%$ collodion was painted on one side of glass coverslip followed by drying at oven at $80^{\circ} \mathrm{C}$ for $30 \mathrm{~min}$

\subsection{Buffer Solutions and their importance in the Mobility Assays}

The Buffer solution inside the flow cell plays an important role. Different constituents of the flow cell contribute changes to the environment. The electrolysis process inside the cell due to the electrodes also adds to the process. The buffer performs the function of a balancing agent by maintaining an affable environment for the proteins to survive.Buffers as defined by [16] "a substance which by its presence in solution increases the amount of acid or alkali that must be added to cause unit change in $\mathrm{pH}^{\prime \prime}$. The $\mathrm{pH}$ levels are maintained by the buffer by maintaining a constant source of hydrogen ions within the solution. An acidic buffer solution is simply one which has a $\mathrm{pH}$ less than 7. Acidic buffer solutions are commonly made from a weak acid and one of its salts - often a sodium salt. An alkaline buffer solution has a $\mathrm{pH}$ greater than 7. Alkaline buffer solutions are commonly made from a weak base and one of its salts. The buffer solutions that are employed in the field of biochemistry usually satisfy the following properties [17]

1. Solubility in water

2. No interference with biological processes

3. Non toxic

4. Non interference with biological membranes

5. Known complex forming tendency with metal ions 
The main function of a buffer is to maintain a constant $\mathrm{pH}$ and thereby guarding the biological specimens, in this case - actin, against variations in $\mathrm{pH} . \mathrm{pH}$ of a buffer solution can be found using the Henderson-Hasselbach equation

$$
p H=p K a+\log \frac{[\text { base }]}{[\text { acid }]}
$$

All acids and bases have a conjugate acid (HA) and a conjugate base $\left(\mathrm{A}^{-}\right)$. At $[\mathrm{HA}]=\left[\mathrm{A}^{-}\right]$ the $\mathrm{pH}=\mathrm{pK}$. The buffer resists change in $\mathrm{pH}$ maximally at the $\mathrm{pK}$ and the buffering zone is considered to be $1 \mathrm{pH}$ unit above and below the $\mathrm{pK}$. So at $\mathrm{pH}$ below $\mathrm{pK},[\mathrm{HA}]>\left[\mathrm{A}^{-}\right]$ and at $\mathrm{pH}$ above $\mathrm{pK},[\mathrm{HA}]<\left[\mathrm{A}^{-}\right]$. The titration curves of the acid vs bases will give us an idea of the buffering zone. The following plot indicates the titration of a weak acid indicating its ionization and buffering property.

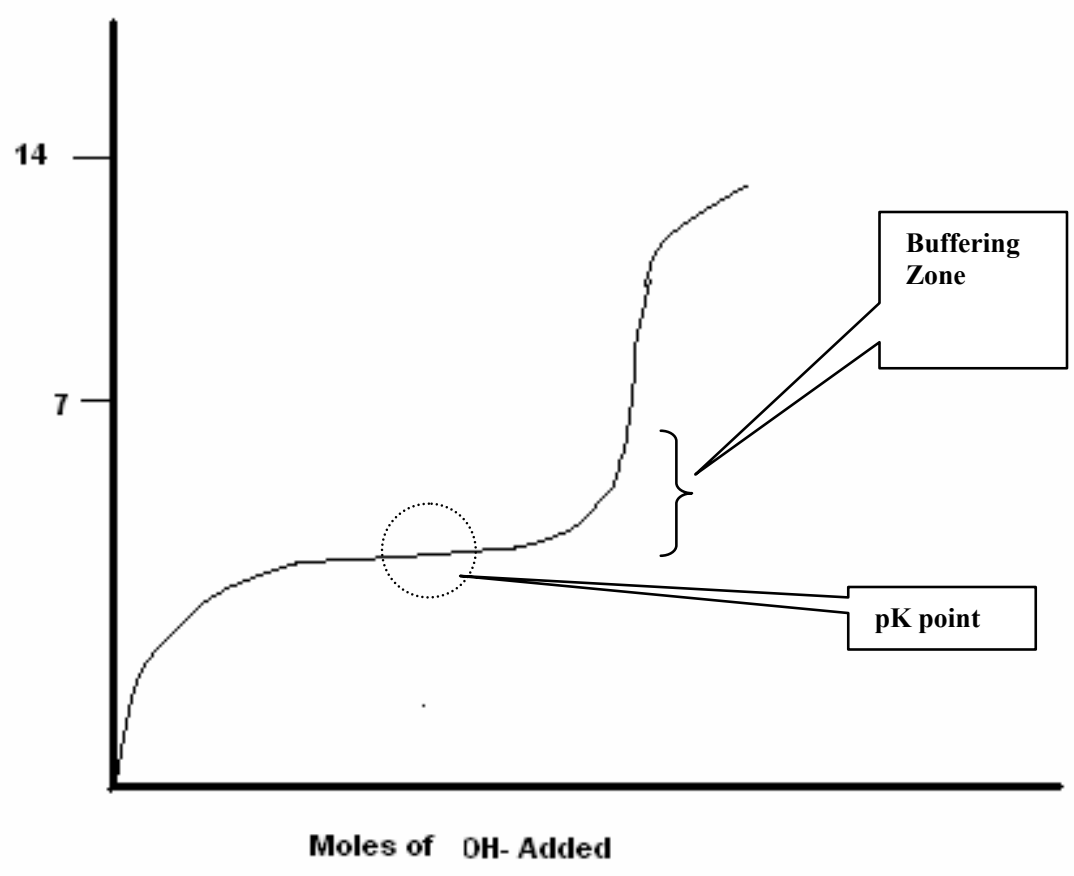

Figure 57 Titration curve of a weak acid 
The above curve is an example of acetic acid ionization. Acetic acid ionizes in the acidic portion of the $\mathrm{pH}$ scale. The position where the buffering zone is on the $\mathrm{pH}$ scale is related to the chemical nature of the weak acid.

$$
H A<=>H^{+}+A^{-}
$$

The $\mathrm{pH}$ is given by

$$
p H=p K+\log \frac{\left[A^{-}\right]}{[H A]}
$$

the $\mathrm{pK}$ of the weak acid is the $\mathrm{pH}$ when $\left[\mathrm{A}^{-}\right]=[\mathrm{HA}]$.

\subsubsection{Amino acids and Isoelectric points}

Proteins structures primarily contain amino acids. For example the myosin head contains 850 different types of amino acids.

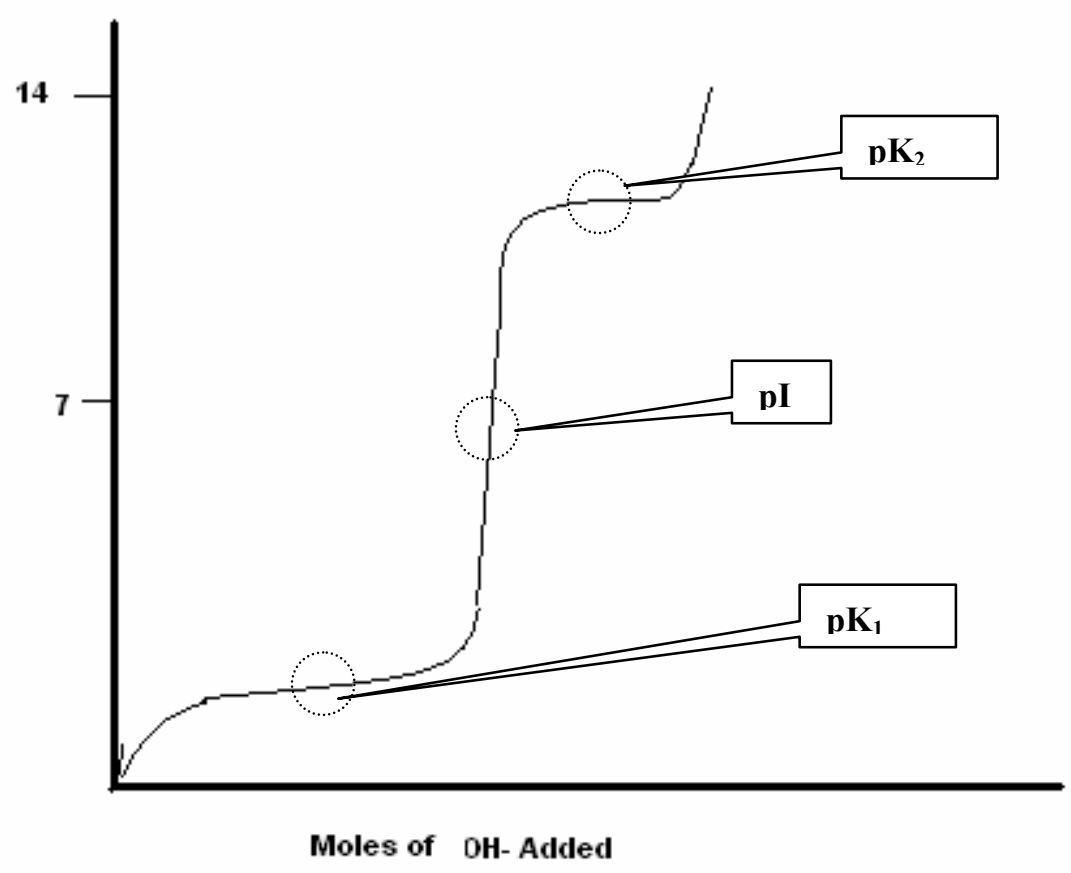

Figure 58 Titration curve of amino acids 
There are at least two different ionizing groups in amino acids and that makes the situation more complex than simple buffers. Since there are two different ionizing groups there will exist two or more values of $\mathrm{pK}$. The following plot illustrates the point. The above curve is a titration curve representing the different stages in the case of ionization of amino acids. For example if we consider the case of Glycine (Gly)

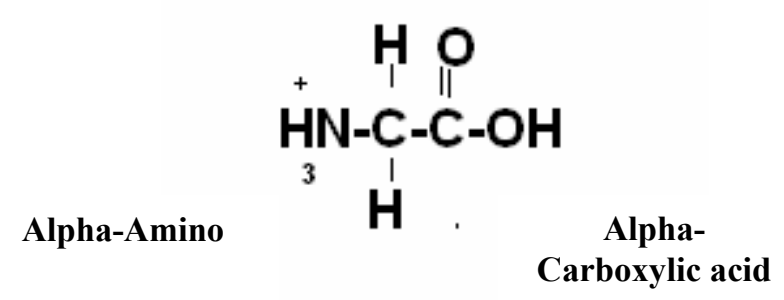

If the acid is dissolved in water it will form a Zwitterion which has both the +ve and -ve groups.

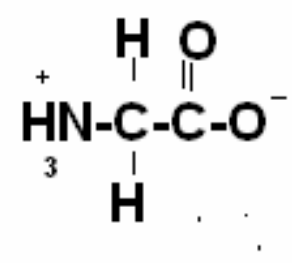

This is still neutral since the +ve charge is balanced by $-v e$ charge. Glycine is a zwitteron in neutral $\mathrm{pH}$. Now if Glycine is put at an acid $\mathrm{pH}$ where it will be fully protonated (i.e. it has all the protons bound to it which it can bind), it can then be titrated to obtain its $2 \mathrm{pK}$ values for the alpha-carboxylic acid group and the alpha amino group. The $\mathrm{pI}$ is the point 
where the glycine is neutral. The pI point is also known as the isoelectric point where the amino acid has no net charge.

$$
p I=\frac{p K_{1}+p K_{2}}{2}
$$

The protein actin also contains amino acids, so following the principles described above the isoelectric point of actin can also be estimated. The isoelectric point of G-actin is reported to be around $\mathrm{pH}$ of 5.06-5.27[18]. This means that the actin was +ve charged below this $\mathrm{pH}$ i.e. at the acidic $\mathrm{pH}$ and has no charge or is neutrally charged at the isoelectric point; has a negative charge at $\mathrm{pH}$ 's above 5.27. Hence at the $\mathrm{pH}$ of the buffer solution i.e. at $\mathrm{pH} 7.2$ the actin filaments are negatively charged.

\subsubsection{Variations in the flow cell}

The flow cell can be the visualized as an electrolytic cell wherein an electrolysis process takes place. In an electrolysis process there are chemical changes within the flow cell due to the flow of current. At the positive electrode (the anode), oxidation occurs and at the negative electrode (the cathode), reduction occurs[19]. The chemical changes that occur at the electrodes can be described by the equations:

$$
\begin{gathered}
2 \mathrm{H}_{2} \mathrm{O} \Leftrightarrow \mathrm{O}_{2}+4 \mathrm{H}^{+}+4 \mathrm{e}^{-} \text {(anode) } \\
2 \mathrm{H}_{2} \mathrm{O}+2 \mathrm{e}^{-} \Leftrightarrow \mathrm{H}_{2}+2 \mathrm{OH}^{-} \text {(cathode) }
\end{gathered}
$$

These chemical changes in the flow cell also affect the $\mathrm{pH}$ of the buffer. The relative electrophoretic velocities of the analytes can be altered by a change in ionization state as a result of $\mathrm{pH}$ change [20]. Electrolysis of water and the resulting $\mathrm{OH}^{-}$and $\mathrm{H}^{+}$produced can change the $\mathrm{pH}$. The change in $\mathrm{pH}$ can cause changes in the attachments of proteins and their segregations. It was observed that at lower $\mathrm{pH}(6.5)$ the actin filaments bundled 
up together and at higher $\mathrm{pH}(8.4)$ the actin filaments were segregated. .This segregation and aggregation of actin filaments with changes in $\mathrm{pH}$ can be attributed to the hydrophobicity of actin. At lower $\mathrm{pH}$ the buffer solution tends to be dehydrating which causes the actin to bundle up; at higher $\mathrm{pH}$ the buffer solution tends to be basic and so the actin remains separate. As more current flows through the buffer solution there is an increase in the chemical changes causing a change in $\mathrm{pH}$. This is due to the fact that the redox reactions imbalance the buffering capacity of the buffer solution.

\subsection{Protocol}

\subsubsection{Flow cell setup}

A flow cell is the chamber/area under concentration where the experiment is carried out. The flow cell was set up on the centre of the slide. In case of a micro electrode structure the flow cell was set so that all the electrodes are under the flow cell chamber. In case of the chrome big electrodes the flow cell was set up on the gap between the big banks of electrodes. Care was taken to see that the gap remained at the centre of the flow cell. The flow cell was set up using a coverslip (18mm x $18 \mathrm{~mm})$. The cover slip was coated with nitrocellulose. The coverslip was fixed in the area of concentration using a double sided tape. The tape had adhesion on both the top and bottom sides. The tape was first adhered on to slide initially and then the coverslip was placed on the top. The coverslip was tapped delicately on the top to get a decent adhesion onto the tape. The tape served as a spacer. The height of the coverslip from the glass slide was around $100 \mu \mathrm{m}$, which is roughly the height of the tape. Hence this acted as a spacer. The buffer solution, motility solution were introduced into the flow cell with a micro injection. As mentioned earlier 
the size of the glass slide is $76 \times 26 \mathrm{~mm}^{2}$. The block diagram of the setup looked like the one below.

\subsubsection{Perfusion of respective solutions to flow cell between glass coverslip and glass slide in room temperature $\left(23-26^{\circ} \mathrm{C}\right)$}

The solutions were introduced in the flow cell using a micro injection. The solutions were introduced one at a time accordingly. For an assay with only Actin the step 1,2 were skipped.

1. Myosin or HMM at $35 \mu \mathrm{L}$ for $5 \mathrm{~min}$

2. BSA solution at $40 \mu \mathrm{L}$ for $5 \mathrm{~min}$

3. Assay buffer at $50 \mu \mathrm{L}$

4. Actin solution at $50 \mu \mathrm{L}$ without ATP

5. Actin solution at $50 \mu \mathrm{L}$ with $1.5 \mathrm{mM}$ ATP

A graphical illustration of the process is shown below for a chrome slide

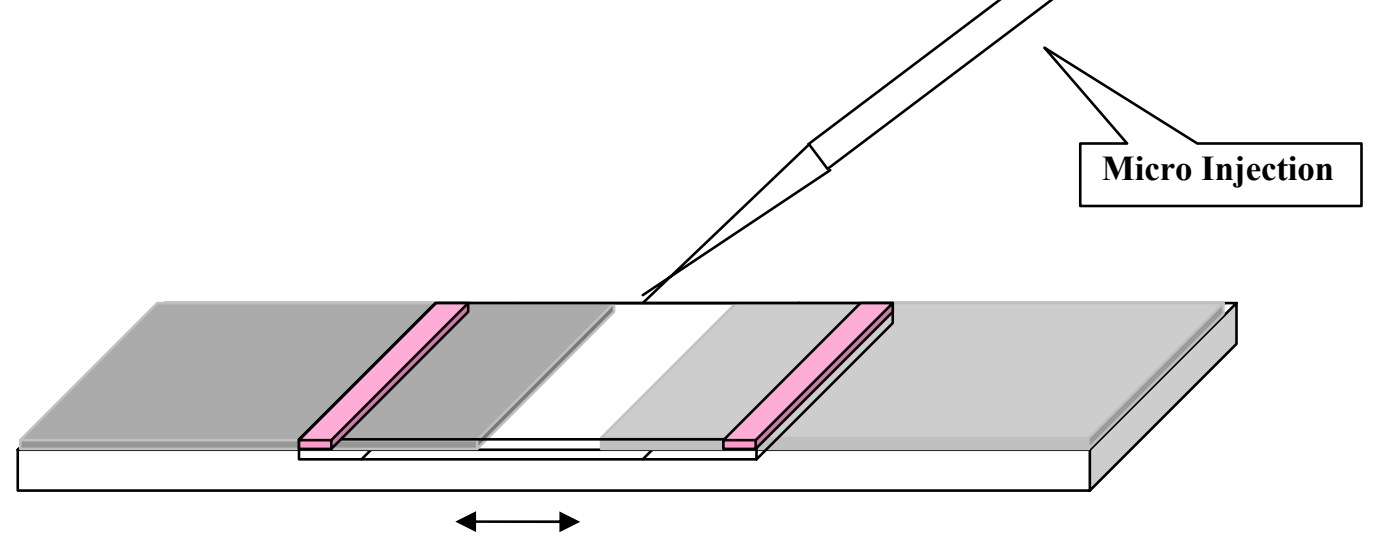

Figure 59 Process of injection of the buffer solutions, actin and HMM

\subsubsection{Insulation of the slide}

For the chrome slide, since there were two big banks of electrodes additional insulation was required at the ends. This was because of the fact that the slide holder of the 
microscope was metallic and the pedestal or the floor of the microscope was also a non insulating one. As a precautionary measure to prevent short circuiting of the slide when the voltage was applied, additional insulation was needed at the point of contact of the holder and the slide and the floor and the slide. An insulating tape was used for this purpose. The insulating tape was wound on the sides on the slides. This also served to hold the electrical connection wires onto the chrome slides. The tapes so wound on the glass slide slightly raised the slide on the floor of the microscope and thus preventing any kind of direct/indirect contact of the two polarities of the applied voltage. The graphical representation of it is as follows.

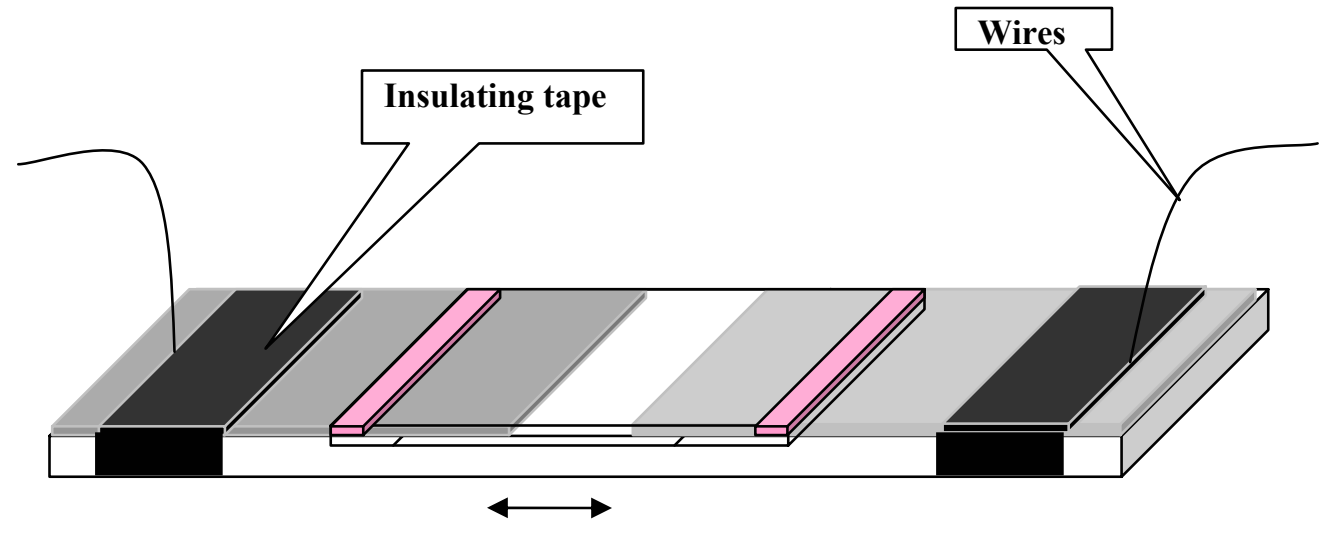

Figure 60

\subsection{Voltage source set}

The Voltage source that was used in this project was a Keithley source meter, model 2400 with measurements upto $200 \mathrm{~V}$ and $1 \mathrm{~A}, 20 \mathrm{~W}$ power output. The source meter can simultaneously be used to measure and supply voltage; it can also measure the current for the supplied voltage. The source meter had a current limit of $105 \mu \mathrm{A}$ when used in the range of $0-5 \mathrm{~V}$. So if the current limit was reached the voltage wouldn't increase further. In such a situation in order to increase the voltage applied the current had to be increased. 
Care should be taken and the current should be continuously monitored to arrive at a stable voltage.

\subsection{Images of the set up}

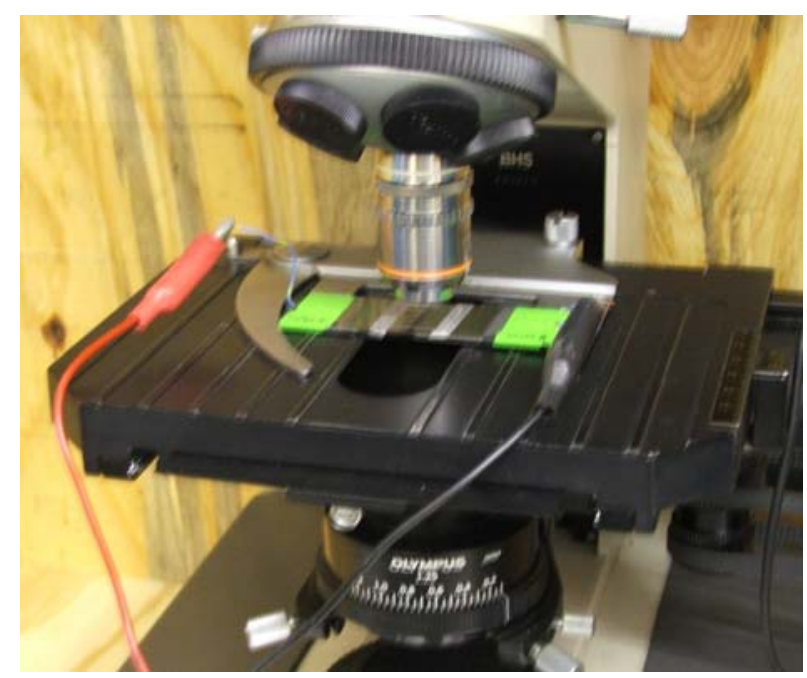

Figure 61 Macro electrode assay setup

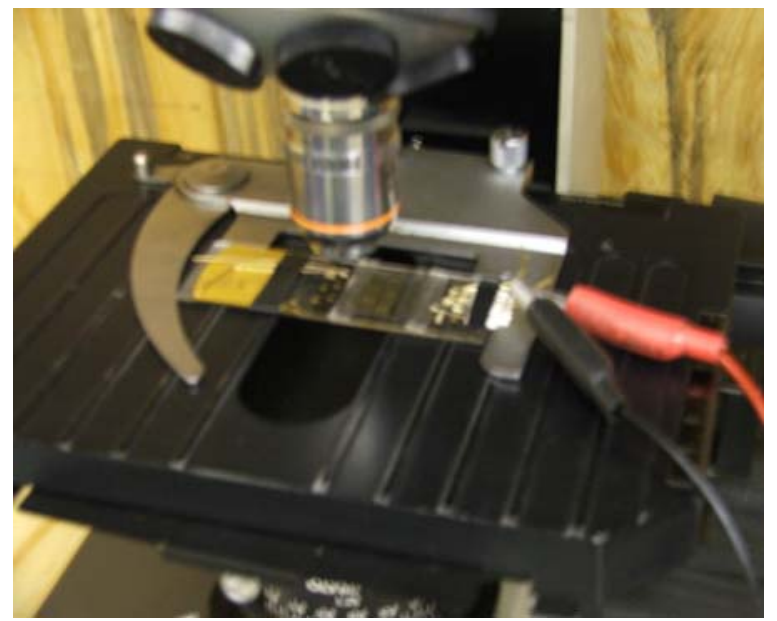

Figure 62 Microelectrode setup 


\section{Chapter 6. Experimental Results and Discussion}

The results of the mobility assays can be characterized as the response of the actin filaments to the electric field. The experiments were carried out under different conditions of

1. Buffer Solution + F-Actin.

2. Buffer Solution + F-Actin + BSA + Collodion.

3. Buffer solution with $\mathrm{KCl}$ concentration of $25 \mathrm{mM}, 60 \mathrm{mM}$ and $100 \mathrm{mM}$.

4. Buffer solution with $\mathrm{pH} 6.5,7.2$ and 8.0.

The voltages applied were primarily determined by the simulation data (Chapter 3). The response of actin filaments with respect to the current passing through the solution was also recorded on a video tape. The video tapes were analyzed for the calculation of velocities. All the videos were at the magnification of 40X.

\subsection{Method of calculation}

The actin filament's response to the electric field was characterized by the velocity of the filament in the flow cell. For the purpose of calculation of velocity the observable region was graduated into 16 parts of $0.01 \mathrm{~mm}$. This graduation scale was chosen with the assistance of a marker. The marker is placed under focus of the $40 \mathrm{X}$ magnification lens. The magnification 40x allowed the visibility of 16 gaps of $0.01 \mathrm{~mm}$ width. Hence the screen was divided into 16 graduations. The graphical representation of the same is as shown in the figure below. The $0.01 \mathrm{~mm}$ represents the actual distance. The length of the 
computer screen used for analysis is $29.3 \mathrm{~cm}$. So each centimeter of the computer screen represented 5.4 microns.

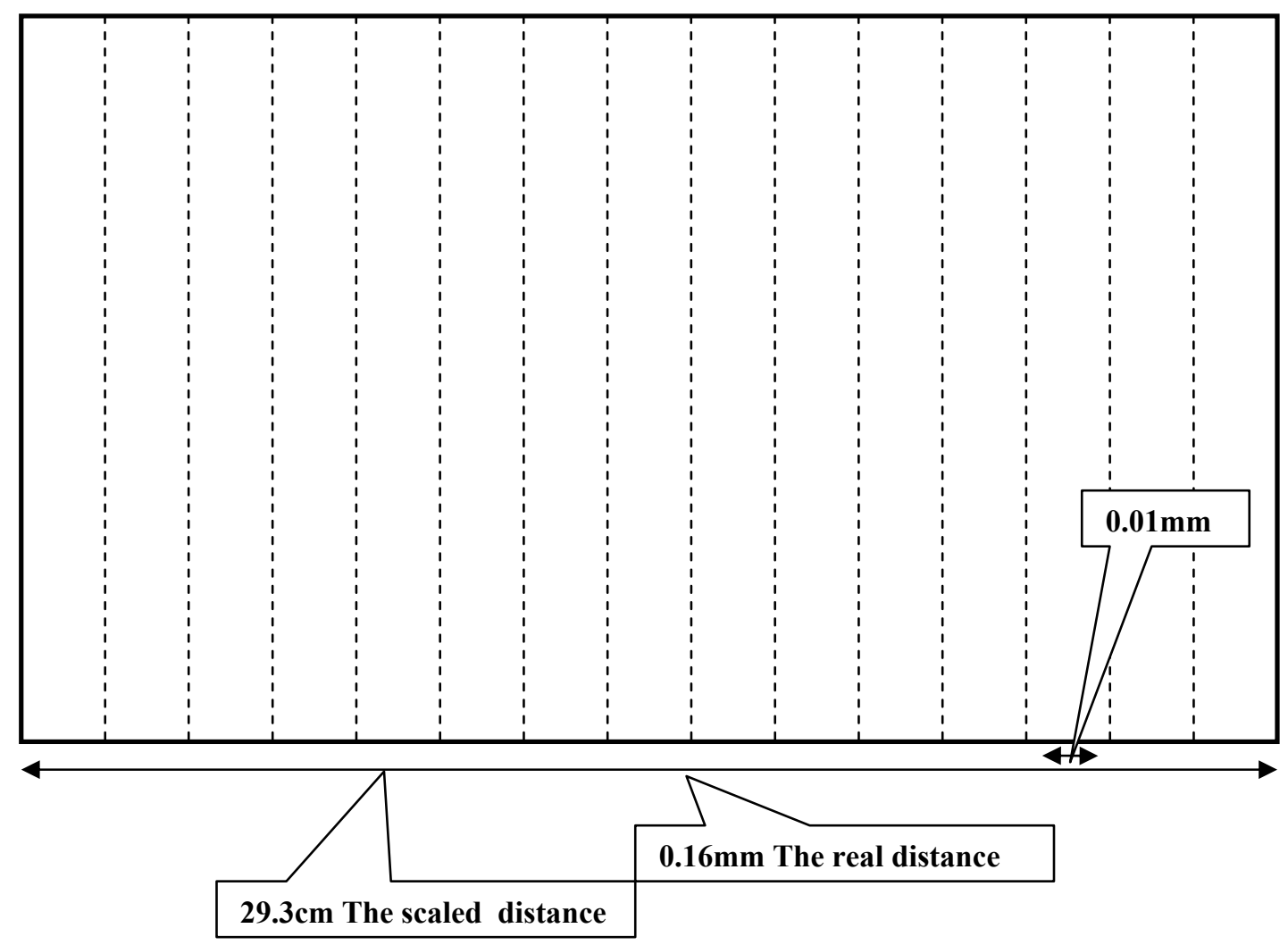

Figure 63 The scaling of the computer screen

The measurement of the velocity takes was undertaken after the scaling parameters were determined. A plastic wrap or a slide was attached to the screen for the mark up of the path of the actin filaments. The markup was done using a marker by following the path of the filament. The actin filaments moved towards the positive electrode when the field was applied. The graphical representation of the mark up is as shown in the figure below. The distance traveled by the filament on the screen and the time taken for it to travel was recorded in a spreadsheet. The distance traveled toward the electrode was divided by the time to get the velocity of the filaments towards the electrode. Since the filaments don't 
always travel in a straight line the displacement of the filament towards the electric field was taken into consideration.

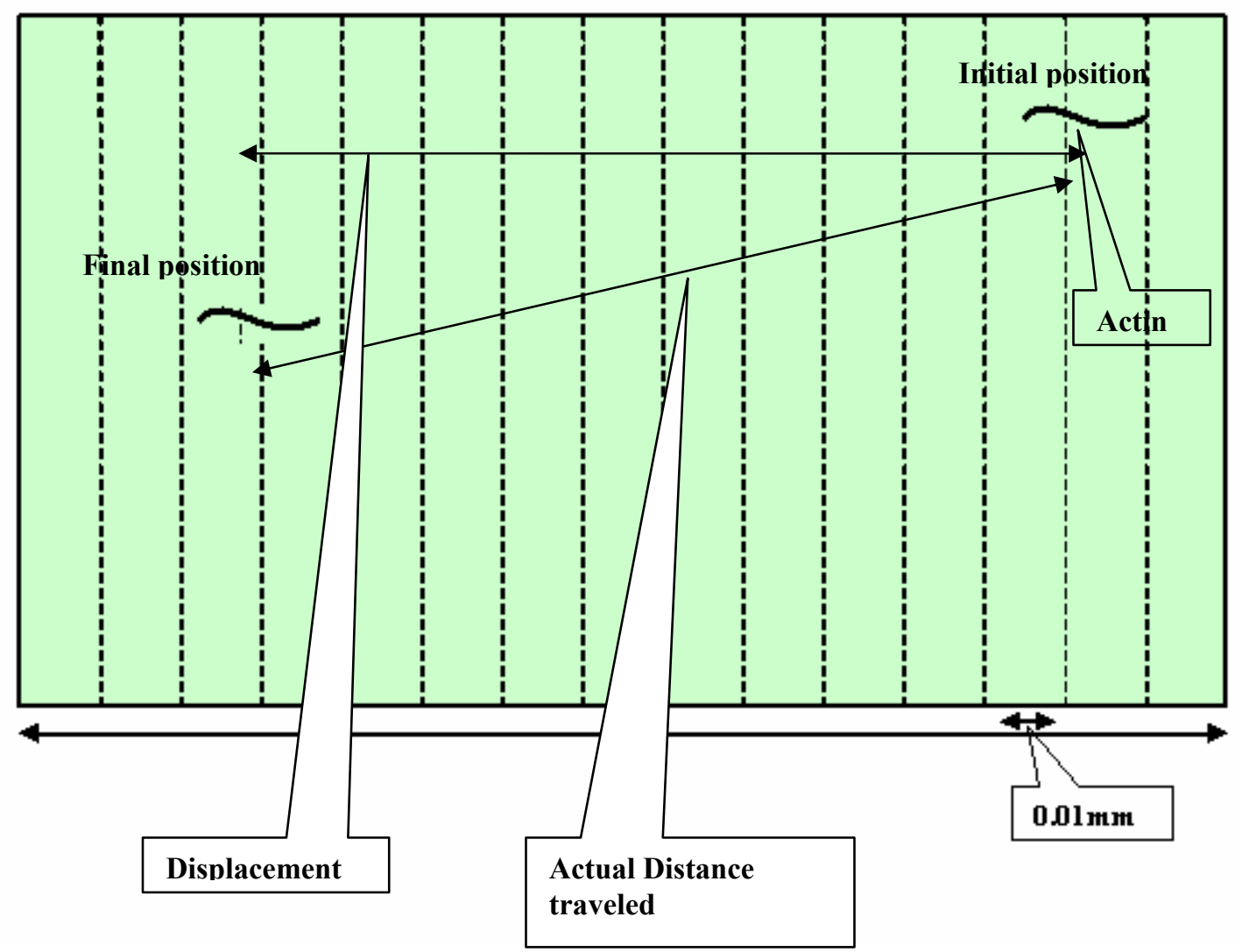

Figure 64 Method of markup of the distance traveled

The displacement was taken into consideration because the travel relative to the field is important. The general motion of actin filament in the absence of electric field is governed by the Brownian motion and micro fluid flows. For considering the movement relative to the field we have to consider the displacement instead of distance traveled. The velocity is measured by scaling the recorded velocities to the original dimensions.

$$
\text { Velocity }=\frac{\text { Dis } \tan c e}{\text { time }}
$$

Ideally the acceleration has to be calculated as per the equation 


$$
\text { Acceleration }=\frac{\text { Velocity }}{\text { time }}
$$

But since the time frame in which the acceleration of actin filament occurs was extremely difficult to determine given the limits of resolution of time, an alternate kinematic approach was taken. The acceleration of the actin filament is calculated from the kinematics equations of

$$
V^{2}-U^{2}=2 \cdot a \cdot S
$$

Where $V$ is the final velocity and $U$ is the initial velocity, $a$ is the acceleration and $S$ is the distance traveled. Since the initial velocity relative to the field is zero the equation gets reduced to

$$
V^{2}=2 \cdot a \cdot S
$$

From which the acceleration can be calculated as

$$
a=V^{2} / 2 . S
$$

Here we make an assumption that the acceleration happens in the smallest resolution we can observe. The smallest resolution is the gap between the lines i.e. $0.01 \mathrm{~mm}$ or 10 microns. So the acceleration is now

$$
a=V^{2} / 2 * 10\left(\text { microns } / \sec ^{2}\right)
$$

The velocity calculations were made for voltages typically between $3 \mathrm{~V}-7 \mathrm{~V}$ for various ambient conditions within the flow cell. The conditions under consideration were mentioned in the beginning of the chapter. 
The following sections will explore the response of the actin filaments taking into consideration the different voltages, currents, different constituents, $\mathrm{pH}$ and their interrelationship with each other.

\subsection{Voltage Vs Field}

The plot of Voltage vs Fields generated from the Simulations is shown below.

\section{Electric field Vs Voltage}

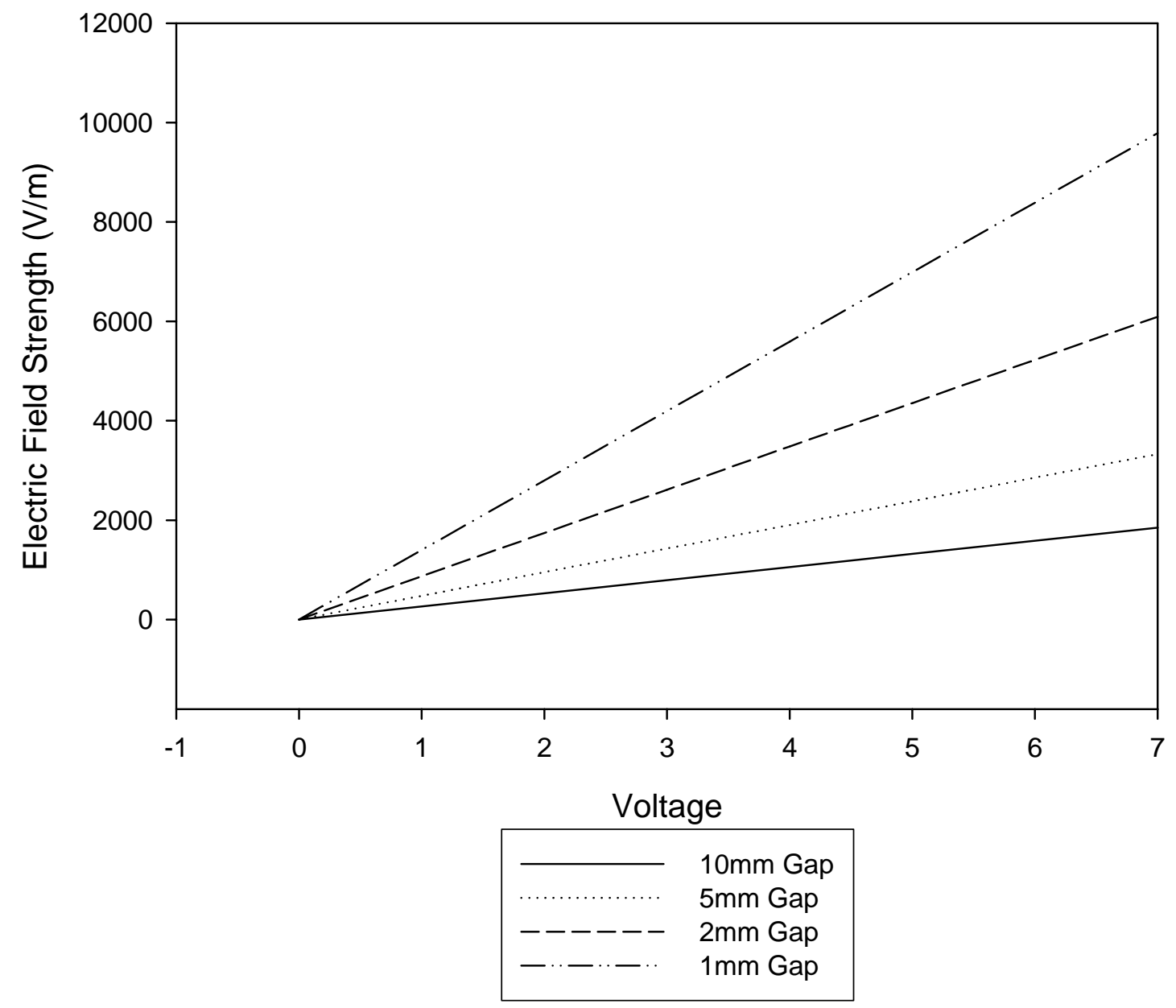

Figure 65 Plot of Electric Field vs Voltage

\subsection{Voltage vs Current}


The Voltage vs Current plot was plotted for various conditions of buffer solution constituents, concentrations and $\mathrm{pH}$ values.

\section{Current Vs Voltage (F-Actin only)}

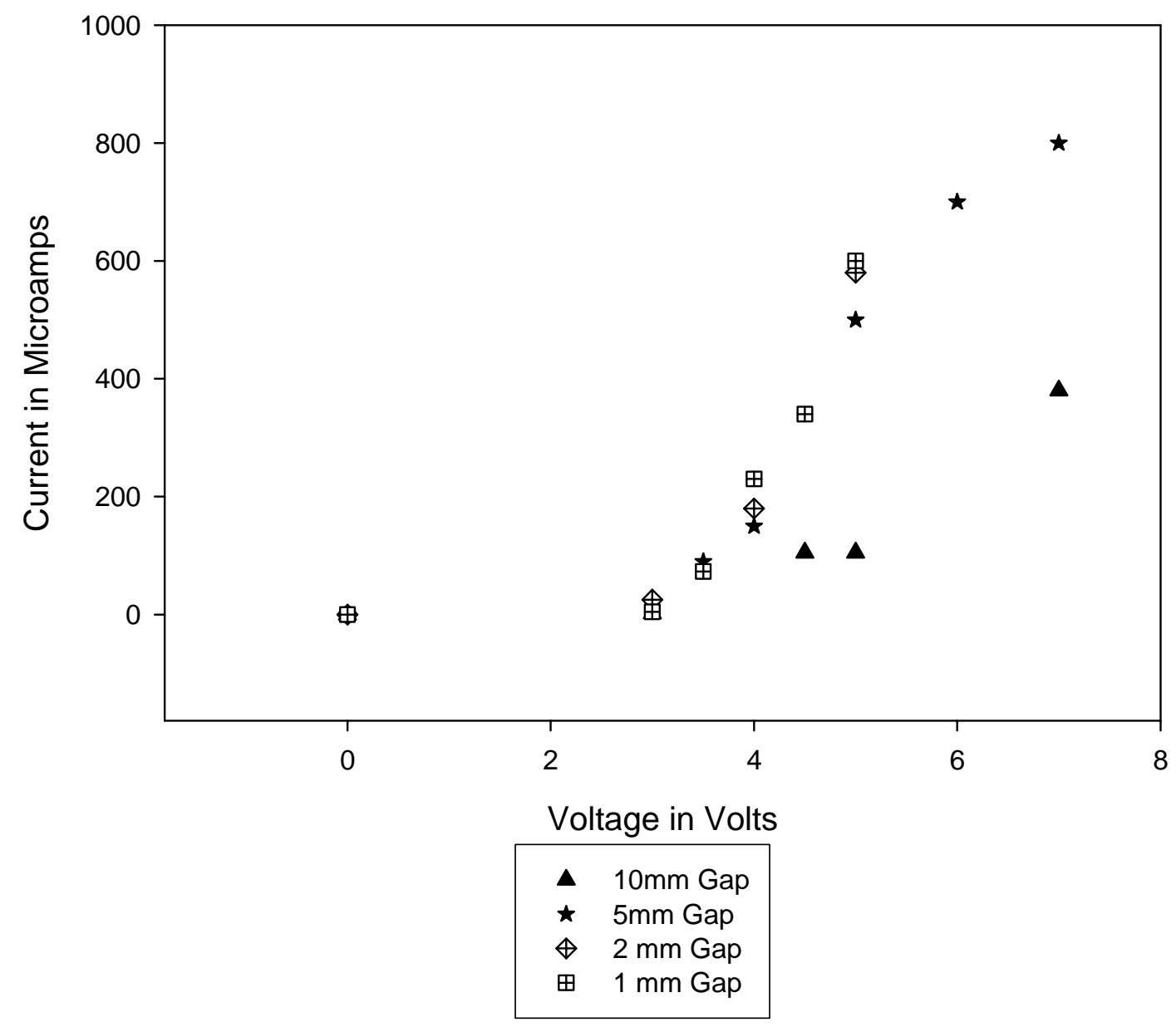

Figure 66 Current vs Voltage in the flow cell with pH7.2, Normal buffer conc, and with only F-actin

The next plot contains the readings when the constituents are F-Actin+ BSA + Collodion. These constituents assume importance in the motility assays which also have HMM in them. Collodion is a membrane and BSA (Bovine Serum Albumin) prevents the 
attachment of actin to the collodion in a motility assay. The plots show that the current readings of for the voltage specified are more or less the same.

\section{Current Vs Voltage (F-Actin+BSA+Collodion)}

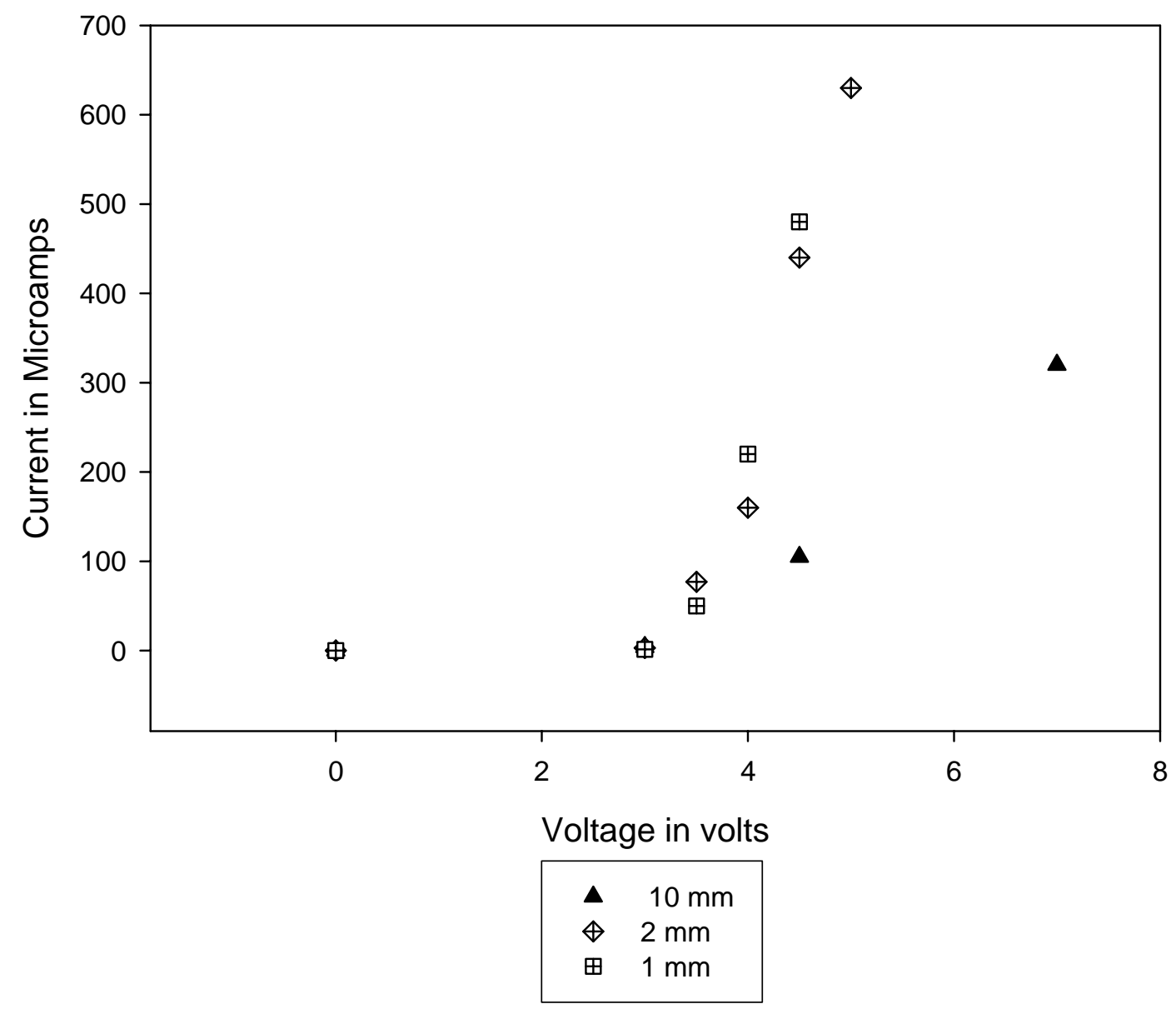

Figure 67 Current vs Voltage in the flow cell with pH7.2, Normal buffer conc, and with BSA+ Factin+ Collodion

The next plot indicates the current flowing through the buffer solution in the flow cell with respect to the different variations in the $\mathrm{pH}$ of the buffer solution. Its observed that though there is more current flowing through the more acidic solution (more $\mathrm{pH}$ ) the current difference is not notably different. 


\section{Current Vs Voltage for different $\mathrm{pH}$}

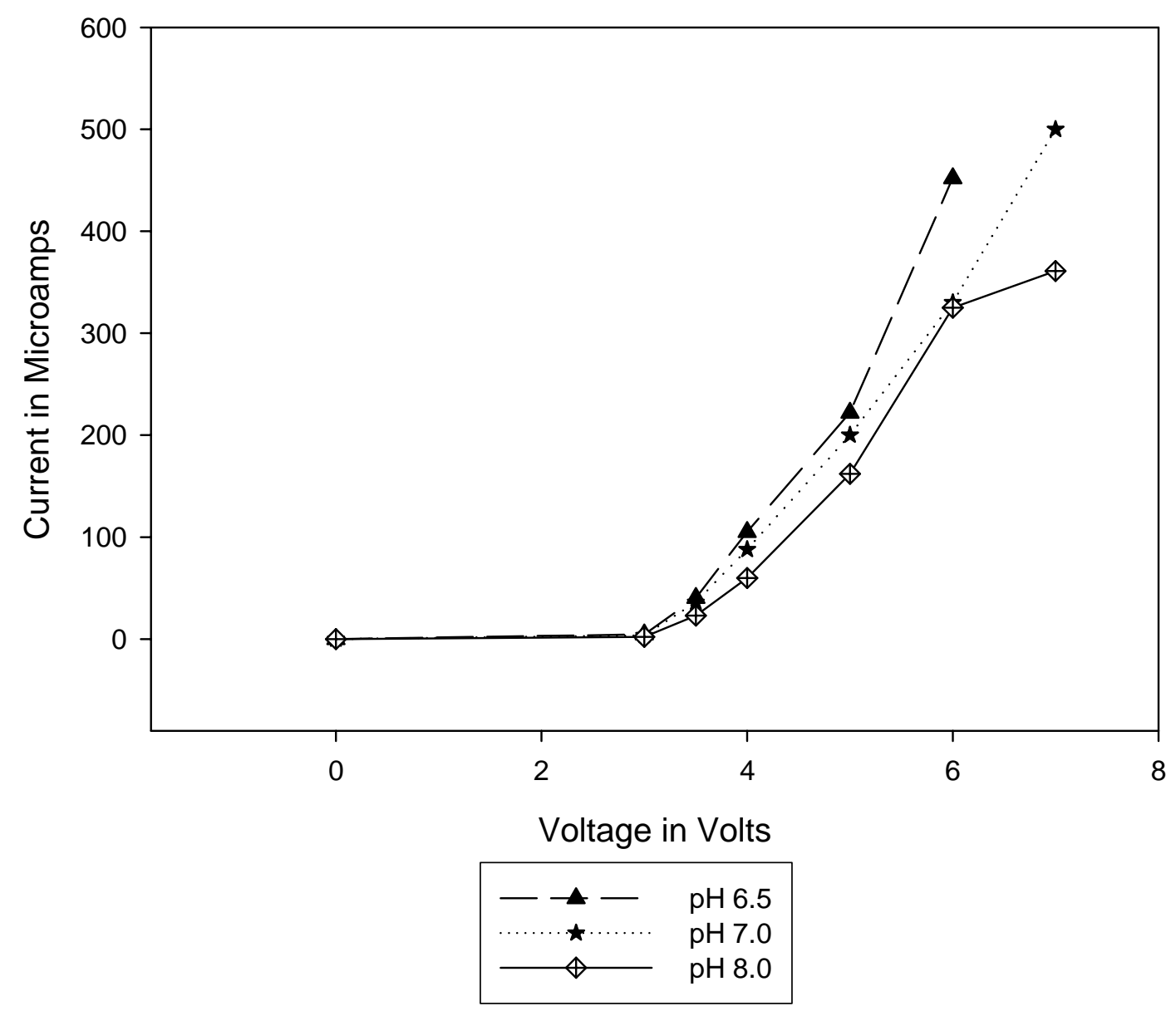

Figure 68 Current vs Voltage in the flow cell with varying pH, Normal buffer conc, and with F-actin

The next plot explores the current flow of in the buffer solution when the concentration of $\mathrm{KCl}$ is different. The concentration of $25 \mathrm{mM}, 60 \mathrm{mM}$ and $100 \mathrm{mM}$ of $\mathrm{KCl}$ in buffer solution can be termed as suitable for existence of actin proteins without denaturing. However the concentration of $\mathrm{KCl}$ in excess of $60 \mathrm{mM}$ can be detrimental in causing denaturing of HMM in case of a motility assay. The plots again show similar current flows, if not the same. 


\section{Current Vs Voltage for Different Buffer Concentrations}

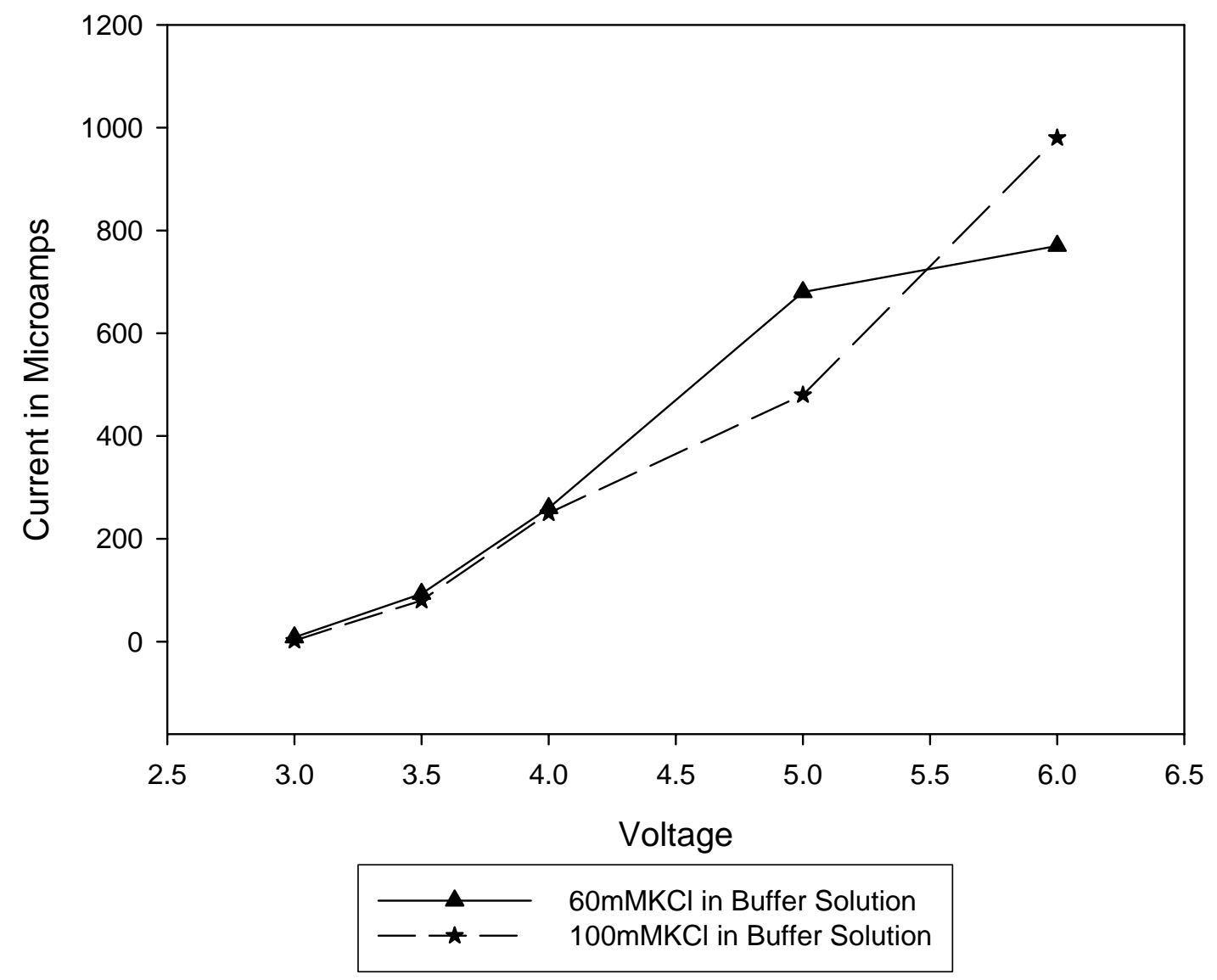

Figure 69 Current vs Voltage in the flow cell with $\mathrm{pH}$ 7.2, and varying buffer conc, and with F-actin

The similar current flow in the cases of variation in $\mathrm{pH}$, variation in buffer solution and variation in constituents predict similar response of actin in varied conditions, although some of the conditions may not be suitable for the motility assay. The motility assay has to be in extremely controlled conditions as the HMM is sensitive to changes. It was observed that the actin was more aggregated at lesser $\mathrm{pH}$ and segregated at greater $\mathrm{pH}$. The aggregation can be attributed to the higher hydrophobic nature of actin at lower $\mathrm{pH}$. 


\subsection{Velocity Vs Voltage}

The voltage response of the action for normal buffer concentrations and a $\mathrm{pH}$ of 7.2 with only actin in the buffer solution is plotted as below.

\section{Velocity versus Voltage(F-Actin Only)}

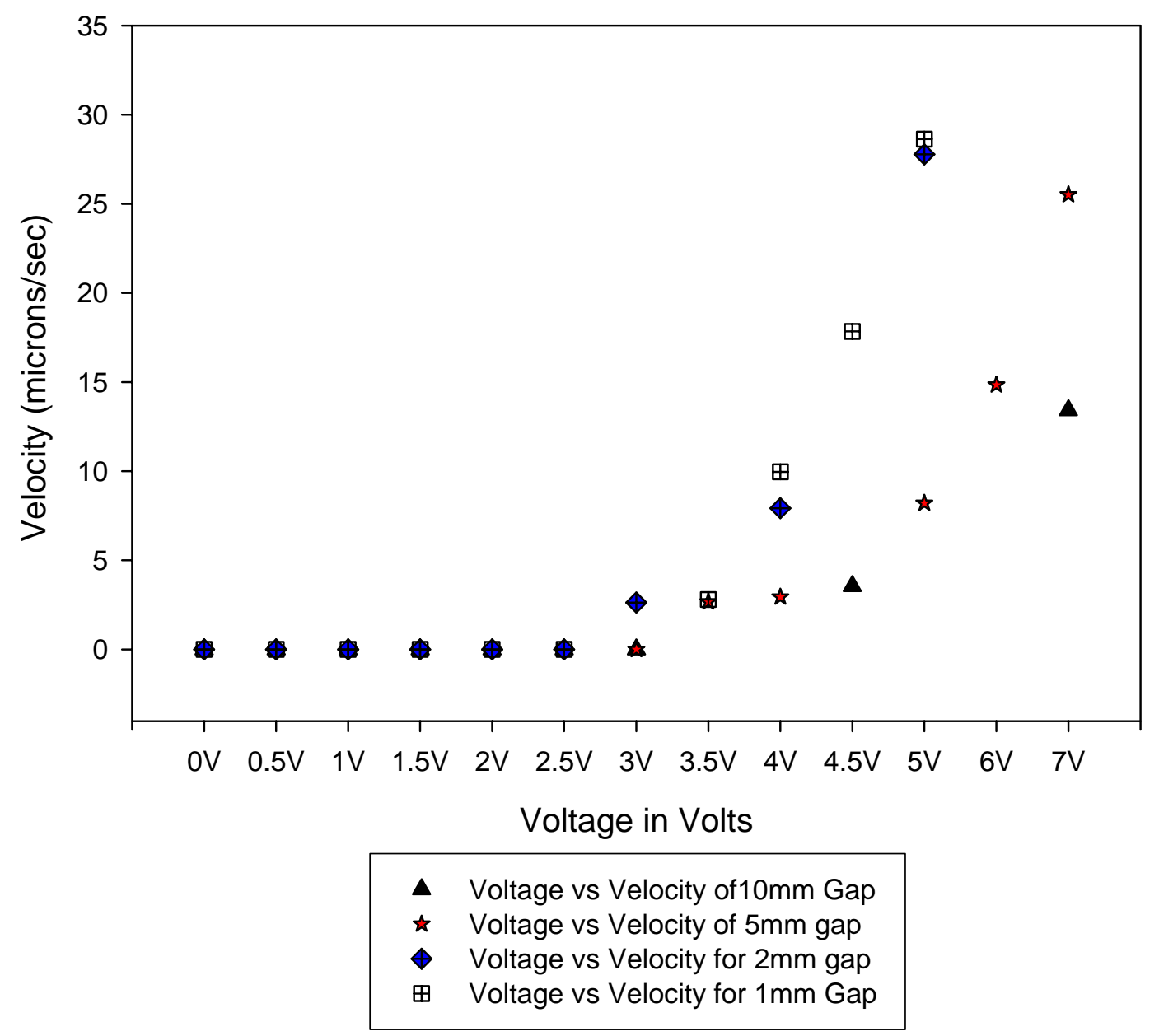

Figure 70 Plot of Velocity Vs Voltage under normal concentration of buffer, pH 7.2 with F-Actin The plot indicates that the actin begins responding to the voltage only at $3.5 \mathrm{~V}$. This voltage corresponds to the starting of the current flow through the buffer solution within the flow cell. The velocity gradually keeps increasing with the increase in voltage. The velocity of actin with different gaps between the electrodes is mentioned. It is observed 
from the plot that the velocity assumes greater values for the same voltages when the gap between the electrodes is narrower. This behavior was expected as the electric field strength between the electrodes increases as the gap gets narrower. The plot with error bars is depicted below. The error bars were a function of the standard deviation.

\subsubsection{Terminal Velocity}

The velocities observed/estimated during the calculations can be termed as terminal velocities of the filaments. Literature pertaining to capillary electrophoresis indicates that the head size of the particles undergoing motion due to the electric fields has little or no effect on their velocities. The head sizes ranging from $1.5 \mathrm{~m}-50 \mathrm{~m}$ usually do not have any effect on the velocity of the particle. Ideally the charged filament's velocity should keep increasing as it moves towards the electrode, because of the increasing field on the filament as it moves towards the positive electrode. But the velocity of the filament assumes a constant state after the initial spurt of acceleration. This constant velocity is due to the fact that the liquid medium which consists of the motility solvents and membranes is a lossy medium. The filament traveling in the medium experiencing the electric field force also experiences a drag force due to the viscosity of the medium. The other factors that might play a role are the transmigration of opposite ions in the buffer solution. These forces of drag cause the velocity to remain constant after reaching a limit governed by the lossy medium. This velocity is commonly termed as Terminal velocity. The definition of terminal velocity as a phenomenon in the macro world is as follows, Terminal velocity: (a) the final speed (usually an impact speed) achieved by an object at the end of its path (where gravity, applied physical acceleration and dragging effects may 
all play a part), and (b) in skydiving, the maximum speed achievable for a given object's atmospheric freefall, at which deceleration due to atmospheric friction counteracts further acceleration due to gravity, and no further acceleration occurs - this is now the more common usage.[12] The mathematical equation for this phenomenon for the macro world is represented as follows.[12]

$$
V_{t}=\sqrt{\frac{2 m g}{C_{d} \mathfrak{I} A}}
$$

$V_{t}=$ Terminal velocity, $m=$ mass of the object, $g=$ gravitational acceleration, $C_{d}=$ drag coefficient, is the density of the fluid and $A$ is the objects cross sectional area.

Velocity Distribution (F-Actin Only)

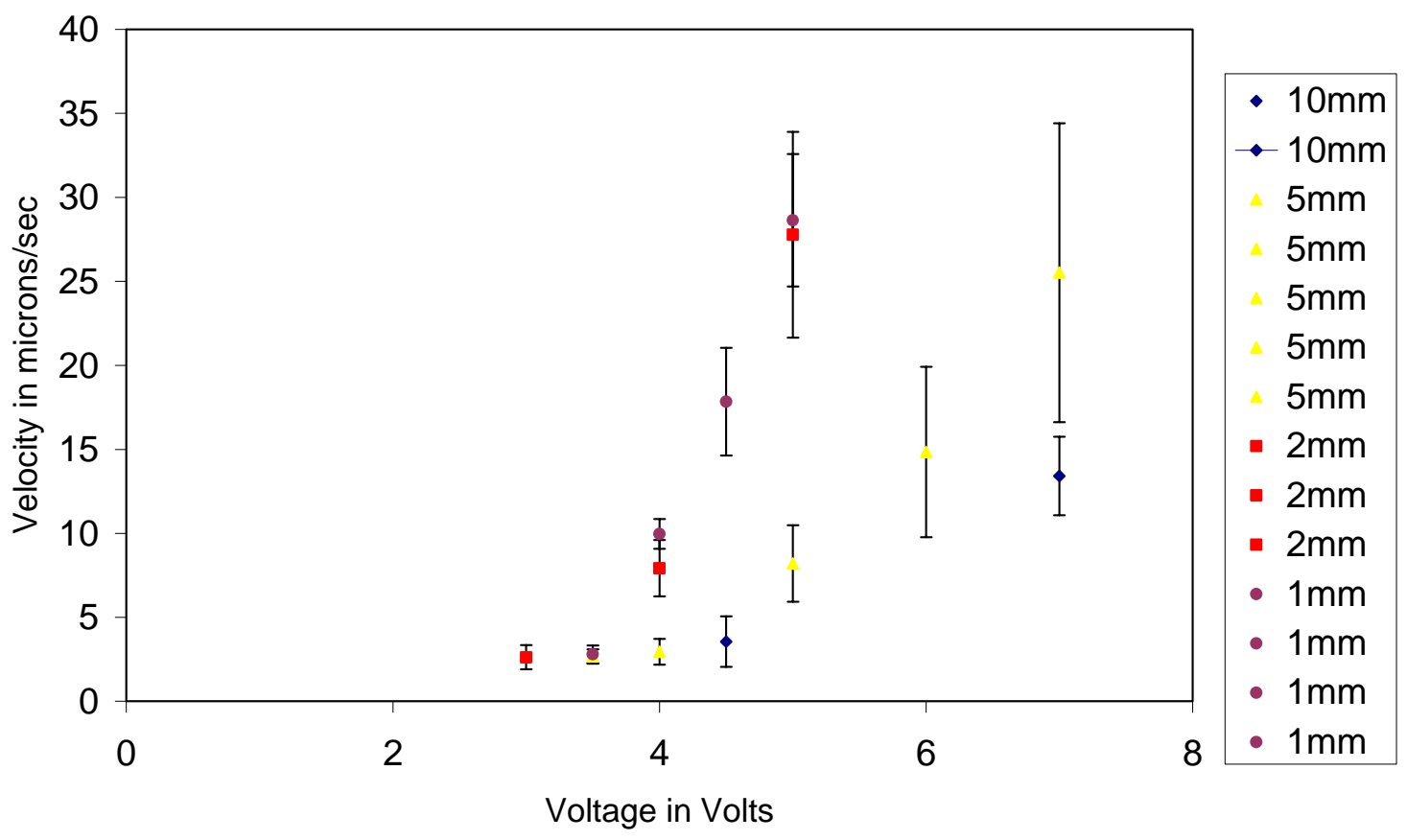

Figure 71 Error bars for the velocity Vs Voltage graph

The next plot has explores the variation of velocity with voltage for different $\mathrm{pH}$ conditions. The actin can survive in the $\mathrm{pH}$ range of 6.4-8.4. The $\mathrm{pH}$ values chosen for these assays were $\mathrm{pH} 6.5, \mathrm{pH} 7.0, \mathrm{pH} 8.0$. The velocity of $\mathrm{pH} 8.0$ started at voltage 3.0V. 
There is marginal difference in the velocities for three different conditions of $\mathrm{pH}$ with $\mathrm{pH}$ of 8.0 showing higher velocity than $\mathrm{pH} 6.5$. It was observed during the assay that the higher $\mathrm{pH}$ of the buffer solution had a segregating effect on the actin filaments, while lower $\mathrm{pH}$ caused the actin to bundle up.

\section{Velocity Vs Voltage with varying pH(5mm gap); F-Actin Only}

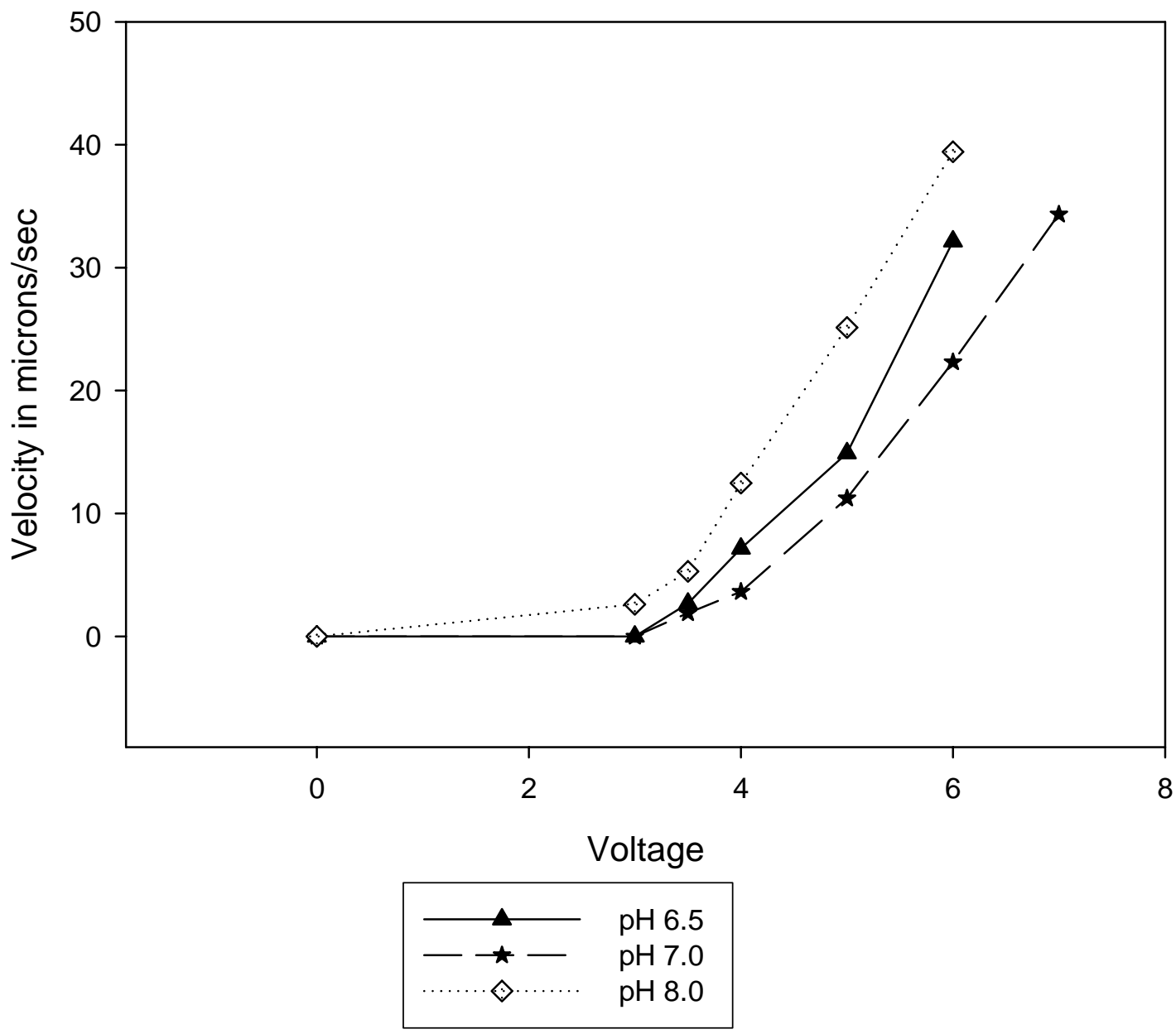

Figure 72 Variation of velocity with $\mathrm{pH}$ for $5 \mathrm{~mm}$ gap, with F-actin only

Though the velocity with varied $\mathrm{pH}$ might not be a precise way of determining the relationship between the velocity and $\mathrm{pH}$, it indicates that a little change in ambient conditions might not alter the velocity by a large degree. A lower $\mathrm{pH}$ (acidic) indicates the 
presence of more $\mathrm{H}_{3} \mathrm{O}^{+}$ions and a higher $\mathrm{pH}\left(\right.$ basic) indicates the presence of more $\mathrm{OH}^{-}$ ions. This by itself does not uniquely specify the conductivity of the solution, because it does not hint anything about the presence of other ions that may affect the electrical conductivity, or about the speed of diffusion. The next plot indicated the velocity response to different voltages for different concentrations of $\mathrm{KCl}$ in buffer solution.

Velocity Vs Voltage (BSA+F-Actin+Collodion)

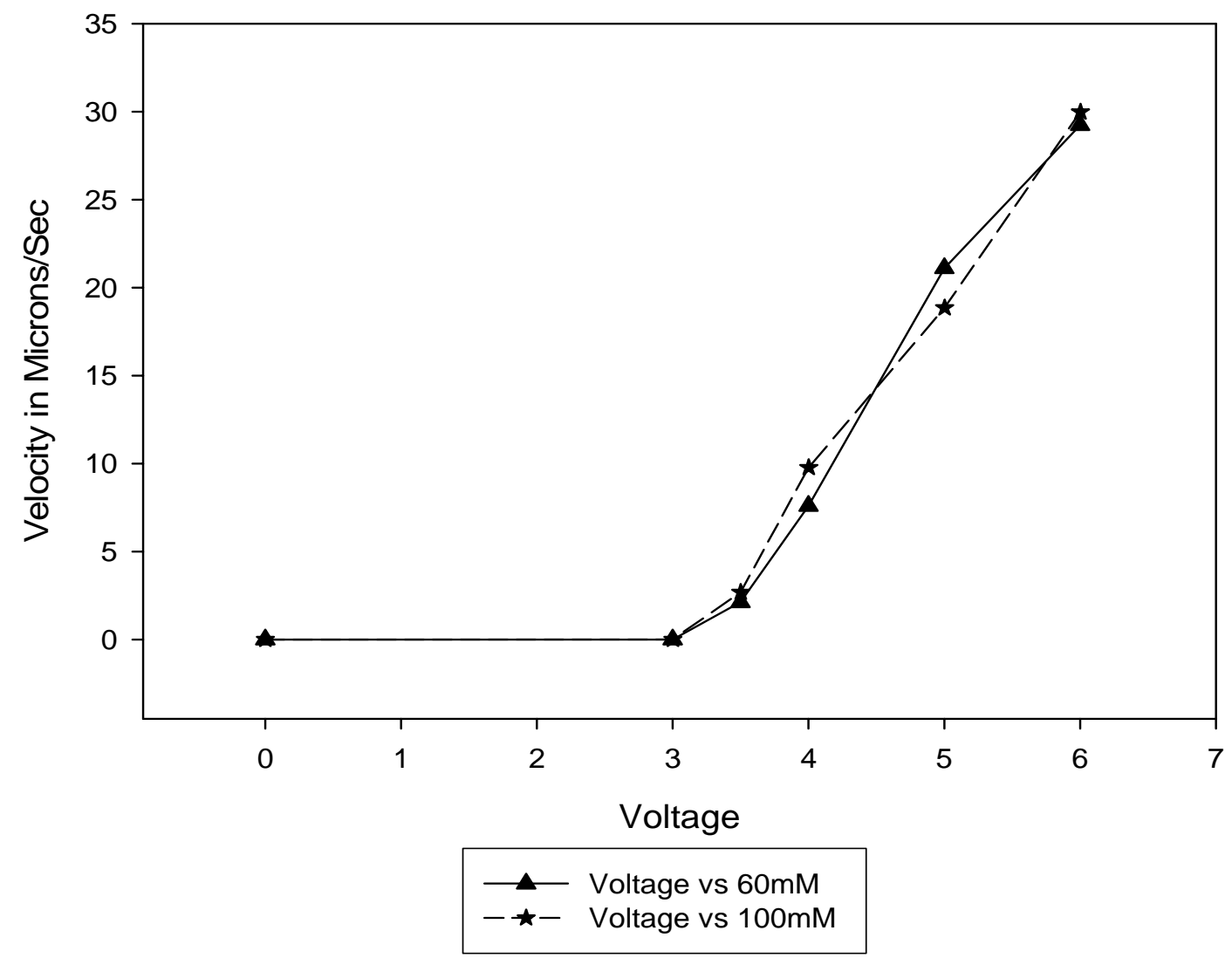

Figure 73 Variation of velocity with buffer concentration for $5 \mathrm{~mm}$ gap, $\mathrm{pH} 7.2$

It can be observed from the plot that the variation in velocity is very marginal, though the concentrations of $\mathrm{KCl}$ were higher. This proves that the velocity of suspended actin doesn't change with the change in concentration of buffer solution. However the 
concentration of $\mathrm{KCl}$ assumes importance in case of a motility assay. A higher concentration of $\mathrm{KCl}$, above $60 \mathrm{mM}$ inhibits the adhering of actin on myosin.

\subsection{Acceleration Vs Voltage}

Under the assumption that the filament, whose average length was empirically determined to be around $10 \mu$, accelerates under the smallest resolution observable i.e. $10 \mu$, the acceleration can be calculated as mentioned in the first section of this chapter.

\section{Acceleration Vs Voltage(F-actin)}

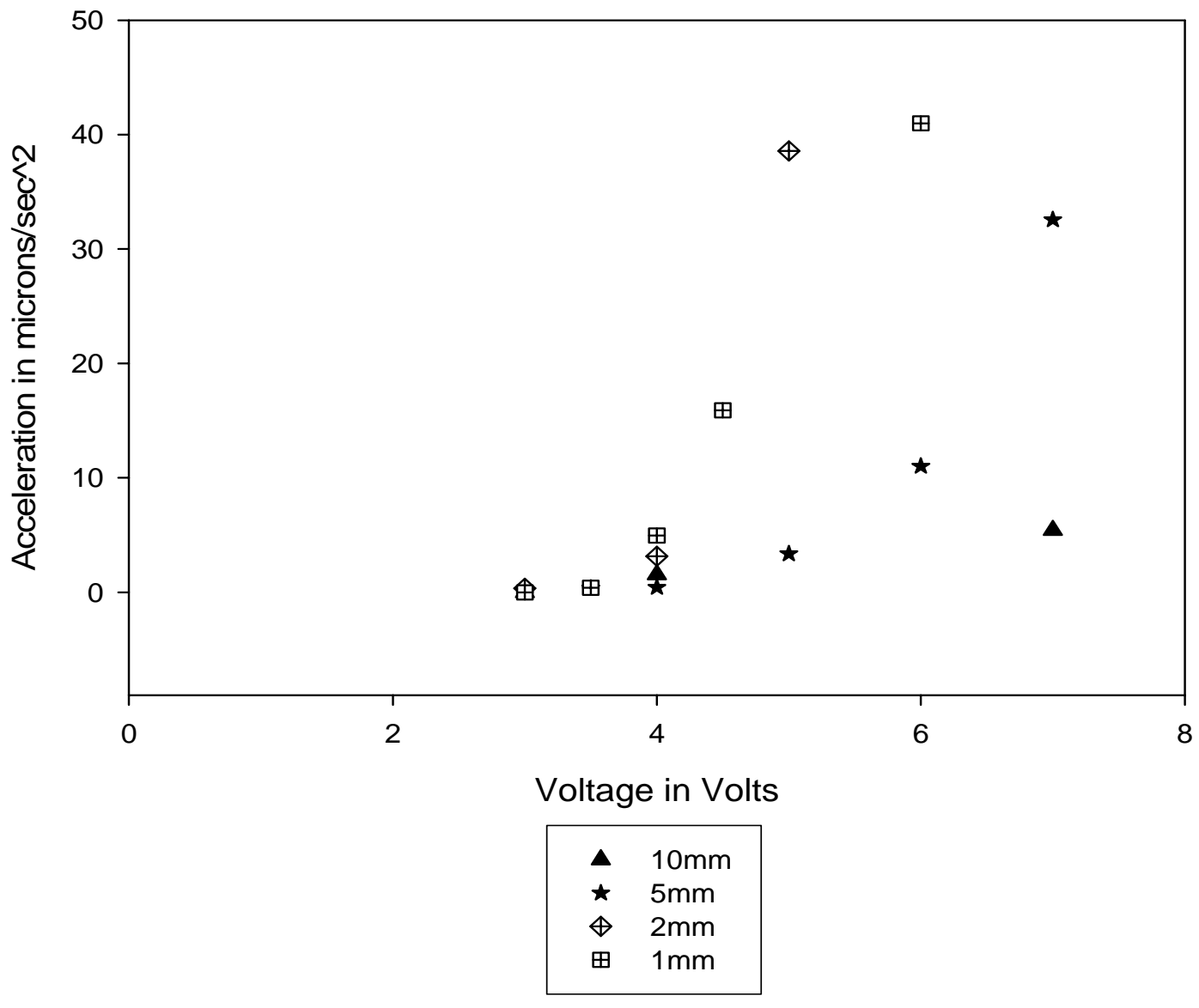

Figure 74 Variation of acceleration in different gaps between electrodes, pH 7.2, F-actin only 
The filament is assumed to accelerate in 10microns. The next plot depicts the acceleration pattern under different voltages for normal buffer concentration, $\mathrm{pH}$ 7.2 As observable the acceleration experienced by the filament increased with the voltage. Another observable point is that the acceleration experienced by the filament with respect to the gap also increases i.e. the acceleration for the same voltage for narrower gap is more than the broader gap between the electrodes. This is complimentary of the trend observed in the Velocity vs Voltage plot for the same condition.

\subsection{Mobility per Electric field}

The mobility per electric field or the mobility is defined as the velocity of the actin filaments per Electric field unit. Mathematically it can be represented as follows

$$
V=\mu E
$$

Where $\mathrm{V}$ is the velocity and $\mu$ is the mobility and $\mathrm{E}$ is the electric field. The mobility is therefore can be written as

$$
\mu=V / E
$$

The plot of the mobility for the voltages applied looks like the plot below. The mobility from the plot though not exactly the same for each voltage, differs by little margin. This can be because of minor errors that could have crept in during the repetition of the experiments. 


\section{Mobility vs Voltage Plot}

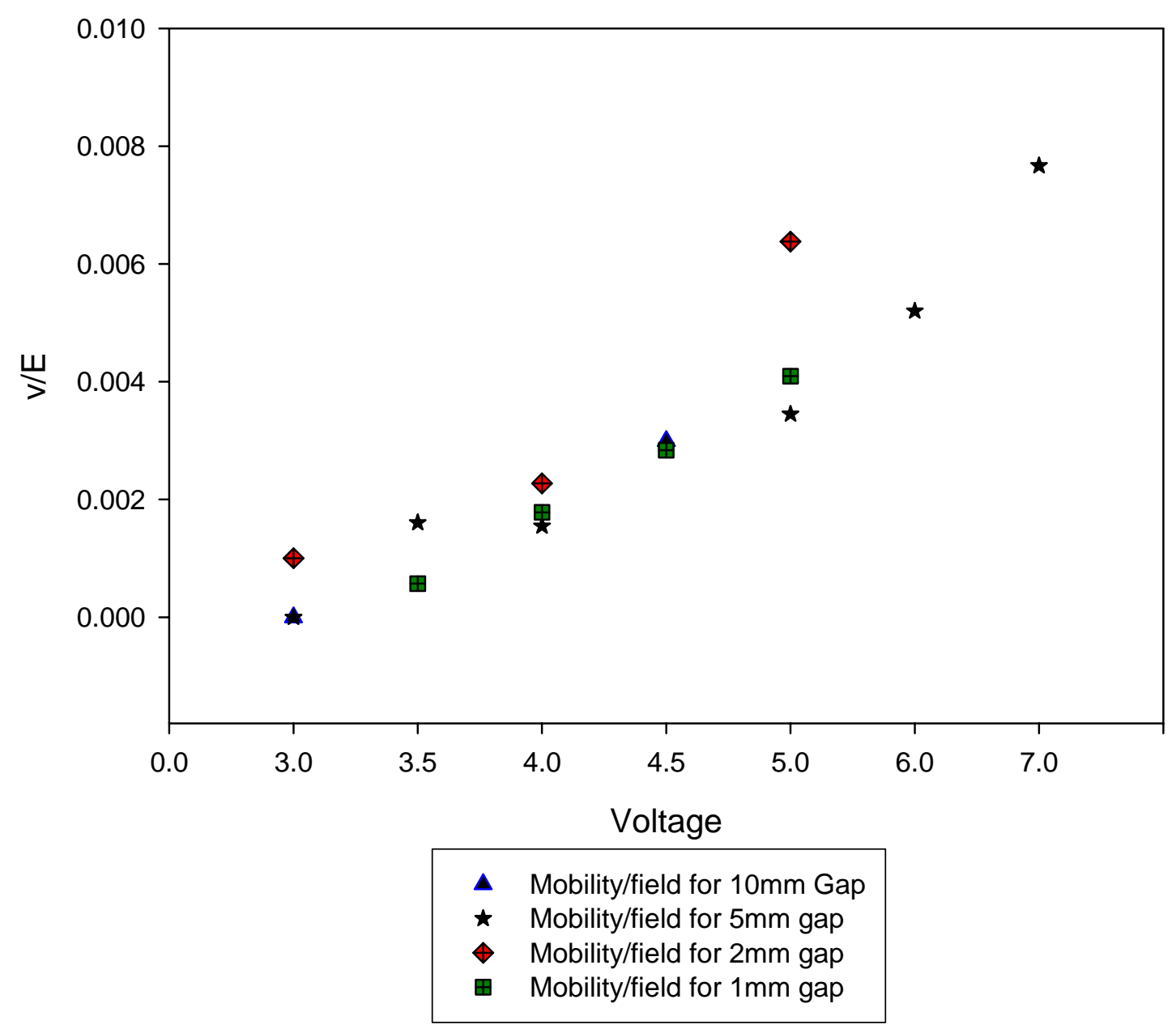

Figure 75 Mobility Vs Voltage plot

\subsection{Lifetime of the Actin Chip System}

The lifetime of the actin system under observation is usually very short, lasting around 15-30 minutes. The lifetime of this experimental system depends on two primary factors, temperature, and photo-bleaching of the system. The temperature of the experimental system can increase due to the heating effects of the current flowing in the system and due to the heating effect due to the fluorescent lamp, which is employed for observation. 
The fluorescent lamp also causes photo-bleaching which renders the dye ineffective after a time period of around 20-30 minutes, this phenomenon is also known as photobleaching. The proteins are maintained in the temperature range of $-80^{\circ} \mathrm{C}$ to $-20^{\circ} \mathrm{C}$ before introducing into the flow cell and the ideal temperature of operation of these proteins is from $25-30^{\circ} \mathrm{C}$. The heating effects denature the protein and therefore the system has to be used within 30 minutes of introducing of proteins. 


\section{CONCLUSIONS AND FUTURE WORK}

The broader perspective of this thesis was to estimate the response of the actin filaments so that the endeavor into the motility assays with Myosin can be characterized accordingly. The response of the naturally charged actin filaments with respect to the electric field was quantitatively estimated with the help of the mobility experiments. The experiments with different gaps between the electrodes and different buffer solution concentrations and different $\mathrm{pHs}$ seemed to indicate the existence of a threshold for the mobility to start. This threshold was primarily thought to be reflective of the ionic conductivity of the solution.

The marginal changes in the ambient conditions altered the velocity of the actin filament marginally. But the response followed a similar pattern. The response of the filaments to the current passing through the solution served as another indicator of the response of the filaments to the electric field. The response under different conditions of $\mathrm{pH}$ and concentrations, constituents provided a base for the motility assays to be characterized.

The reported experiments were carried out on macro electrode structures. The future work on this project can include the attempt to control the directionality of the filaments using

Motility assays with macro electrodes

Motility assays with micro electrodes with inter digitated structures.

Motility assays under the various ambient conditions described in this thesis 
$>$ Pulsed electric fields for more precise directional control.

$>$ Circular electrode motility assays for attaining precise positional control.

Loading and transportation of cargo for micro-transportation.

The implications of the success of this directional control when coupled with the micro cargo handling capacity of actin can be far reaching in the fields of drug delivery and self assembly of materials, enhancing the lab-on-chip technology, to name a few of the seemingly endless possibilities. 


\section{BIBIOLOGRAPHY}

[1] Investigating molecular motors step by step, Jeffrey M Perkel, The scientist, Vol 18, No.5, March 2004

[2] Molecular shuttles based on motor proteins: Active transport in synthetic environments, Henry Hess, Viola Vogel, Dept of Bioengineering, Univ of Washington,Seattle, WA 98195

[3] Acting on actin: the electric motility assay, Daniel Riveline et al, Eur Biophysics, 1998,27,403-408

[4] Molecular mechanism of the contractile cycle of muscle, Sunil Nath, Divya Khurana, Current science, Vol 81, No.1, 10 July 2001

[5] www.ebsa.org/npbsn41/ pictures/myosin.gif

[6] Notes on Brownian motion and related phenomenon, Deb Shankar Roy,Dept. of Physical Chmeistry, Association for Cultivation of science, Jadavpur, December 2001,

[7] Walk of molecular motors in two and three dimensions, T.M. Nieuwenhuizen, S. Klumpp, R. Lipowsky. Europhysics Letters,58(3), pp 468-474, May 2002.

[8] http://www.tiscali.co.uk/reference/encyclopaedia/hutchinson/m0010029.html

[9] http://motility.york.ac.uk/chimaera.shtml

[12] http://en.wikipedia.org/wiki/Terminal_velocity_28disambiguation

[16] Van Slyke, J. Biol. Chem. 52, 525 (1922)

[17]http://www.sigmaaldrich.com/Brands/Fluka__Riedel_Home/Bioscience/BioChemik a_Ultra/Biological_Buffers.html

[18] J.M. Corbett, C.H. Wheeler, M.J. Dunn, Moelectrophoreses of cardiac tissue from human, dog, rat and mouse: towards the establishment of an integrated two-dimensional 
protein database, Electrophoresis 16 (1995) 1524-1529.

[19] Brady and Holum, Chemistry - The study of matter and its changes,1993,

[20] Aaron timperman, Scott E Tracht, and Jonathan V. Sweedler, Dynamic On-Column $\mathrm{pH}$ Monitoring in Capillary Electrophoresis: Application to Volume-Limited Outlet Vials, Analytical Chemistry, Vol 68, No 15, August 1996. 


\section{Appendix A}

\section{A. Process Flow for the fabrication of Gold-Chrome electrodes}

The substrate used in this project was a microscopic glass slide. The dimensions of the glass slide are $26 \mathrm{~mm} \times 76 \mathrm{~mm}$. The thickness of the slide is approximately $1 \mathrm{~mm}$. The microscopic slide was chosen as the substrate for ease of operation with the microscope. Also since the previous experiments on Myosin-Actin motility were conducted on glass slides, the glass slides were used to have uniformity of approach. The glass slides were bought from VWR Scientific Inc, these glass slides came pre-cleaned. The glass slides were frosted at one end. Frost ended slides were chosen to enable easy handling of the slide.

A.1. Substrate Cleaning (slide): The microscopic glass slide was cleaned by immersing the slide in Acetone for $5 \mathrm{~min}$. It is next subjected to an ultrasound bath. The ultrasound bath is used to lift off dirt particles which might have got stuck on to the slide. The slide was then immersed in methanol to de-grease. The slides are then subjected to PiranhaBath clean process which removes any metallic elements present on the slide. The slide is immersed in a Piranha bath for 30 minutes. After 30 minutes the wet slide is then blown dry with nitrogen. The slide was then placed in the oven maintained at $100^{\circ} \mathrm{C}$ for 25 minutes to dry any remaining wetness. 
A.2. Deposition of Metal: The cleaned slides are subjected to metal deposition in an ebeam evaporator. Chrome and Gold are deposited on the slide respectively. Gold doesn't adhere to glass, whereas Chrome has good adhesion to glass. In order to use gold for the electrodes Chrome has to be deposited and then Gold is deposited atop. The deposition parameters will be specified in a subsequent table of parameters. The thickness of the metals deposited was $50 \mathrm{~nm}$ of $\mathrm{Cr}$, and $200 \mathrm{~nm}$ of Au. The e-beam evaporator has a higher vacuum creating capacity than a sputtering station.

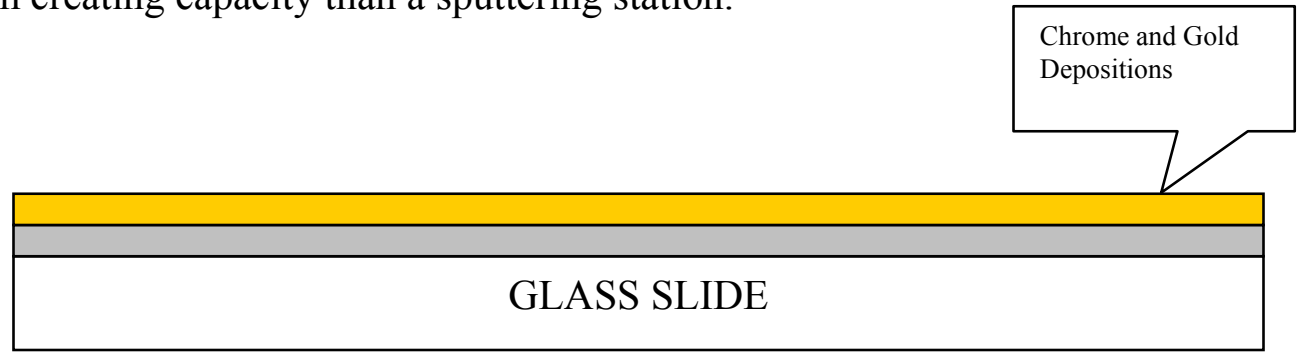

A.3. Photolithography: The metal deposited slide was then subjected to the process of Photolithography. In this process a photoresist was spun on the slide with a spinner. The photoresist used in this process was AZ5214E. With a spin speed of 4000rpm a thickness of $1.8 \mu \mathrm{m}$ of photoresist was achieved. The slide immediately after the spin was soft baked at $90-100^{\circ} \mathrm{C}$ on a hot plate for 1 minute. This was done to let the photoresist solidify and let the wetness dry to an extent. Care was taken not to over bake the photoresist as it hardens the photoresist and adheres more strongly to the surface and can cause problems while developing.

The slide was then subjected to positive photolithography process on a Karl-Suss Aligner. The mask designed for the Au-Cr electrodes was fitted on to the aligner and then the slide was exposed to UV rays. Care was taken to let the pattern take the centre place 
on the slide. Since this process was a positive photolithography process the photoresist areas being exposed to UV rays would be washed during the development process. The photoresist on pattern being blocked by the mask would stay. The UV rays striking the photoresist increases the solubility product of the photoresist and cause it to dissolve when exposed to a developer solution. The exposure parameters are mentioned in a subsequent table. The slide was exposed for $156 \mathrm{~mJ} / \mathrm{cm}^{2}$ as that was the energy that could cause breakage of bonds of photoresist and increase the solubility product.

After the lithography process the slide is subjected to a development process. The developer used is AZ312MIF. The slide was immersed in a 1:4 solution of developer and DI water respectively. The 1:4 ratio was prescribed for better resolution by the datasheets of AZ312MIF developer. The slide was immersed for 2 minutes in the developer solution to get a sharp feature and complete removal of exposed resist. The slide is then rinsed in DI water. This would get rid of undissolved resist. 


\section{Appendix B}

\section{B. Fabrication of Macro Chrome electrodes}

The fabrication of Chrome involves steps which are common to gold chrome electrode fabrication.

B.1. Cleaning of slides: The cleaning of slides is the same process as mentioned in the above section. The cleaning process used was a Piranha bath process.

B.2. Metal deposition: The metal deposition for the big electrodes was done by Sputtering process. The sputtering station was used for this. The sputtering process was carried out at a power of $0.1 \mathrm{KW}$ for 6 minutes. This would give a chrome thickness of approximately $285-290 \mathrm{~nm}$ on the glass slide. The details about the parameters are given in the table.

B.3. Photolithography: The process is very much similar to the process described in the gold-chrome electrode fabrication section. The difference was in the mask used. Here in this process the mask was a pair of black cardboard pieces which were separated at the middle of the slide as per the required thickness. The cross-section of the slide looked like the figure shown below after the spin of the photoresist.

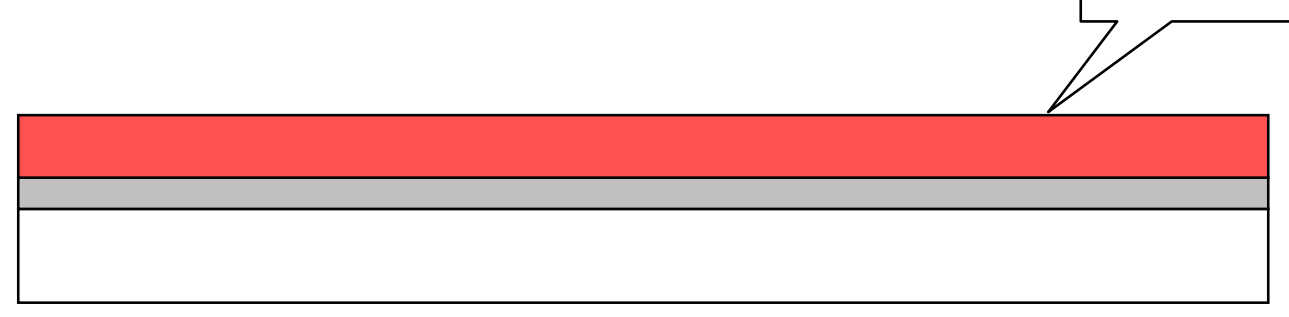




\section{Figure}

The mask on the slide looked like the figure shown below.

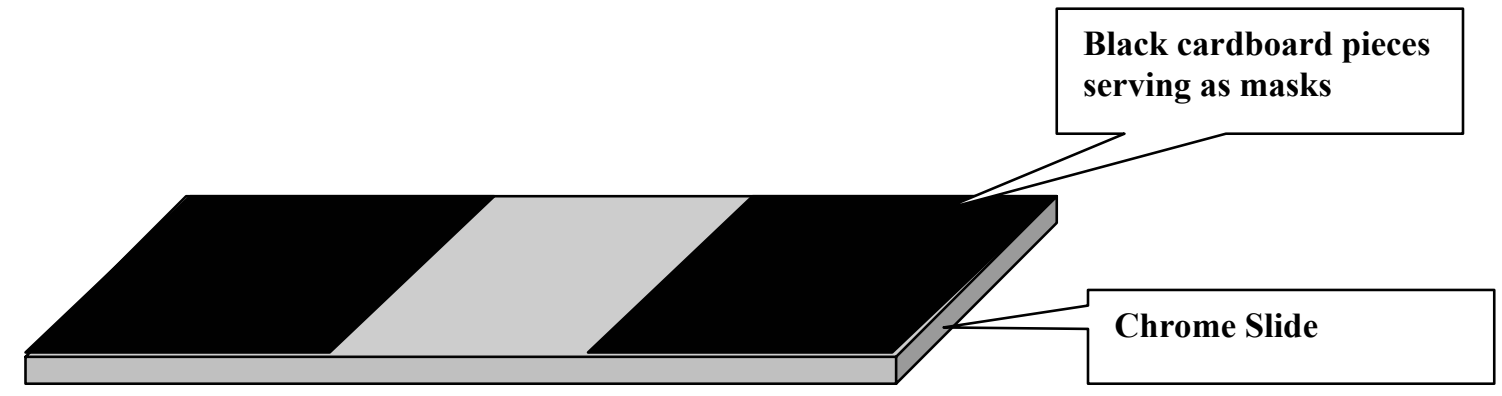

After the exposure and development the slide was subjected to a wet etch of chrome.

B.4. Wet Etching: The slide was immersed in the Chrome Mask etchant for etching of the exposed chrome. The chrome etched in approximately 4 minutes causing a channel that looks like the figure below. The chrome etch process is described in the above section under the wet etching heading. The channel width can be varied by varying the gap between the masks. After etching the channel looked like the figure below.

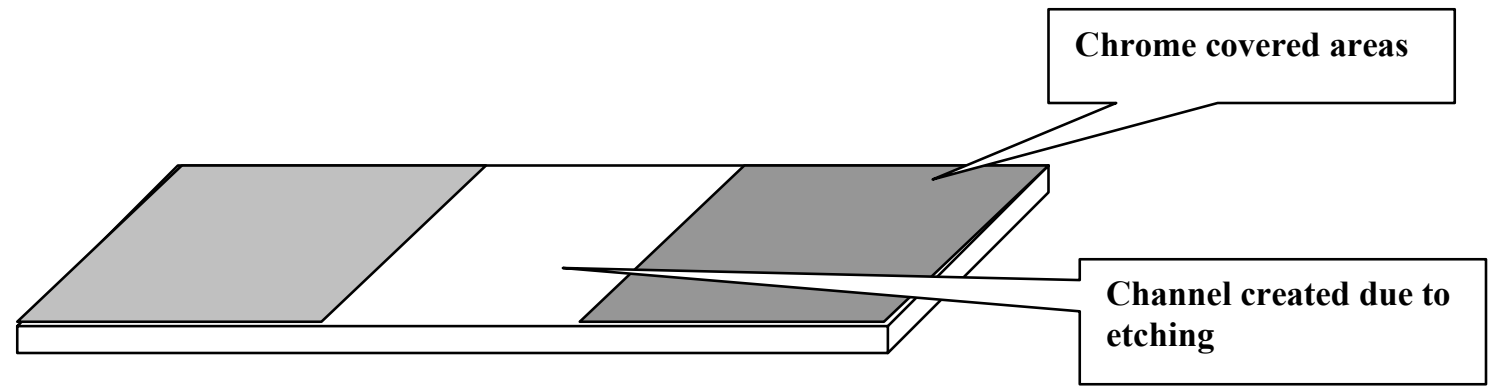

B.5. CVD deposition of $\mathrm{SiO}_{2}$ : An insulating layer of $\mathrm{SiO}_{2}$ was deposited over the chrome slide. The deposition was carried out in a PECVD machine, the details of which are given in the section above. The $\mathrm{SiO}_{2}$ was deposited for 10 minutes causing a thickness of about $0.7-1 \mu \mathrm{m}$. Care was taken to see that some part of the chrome was 
uncovered for the electrical connections. The cross-section of the deposited would look like the figure shown below.

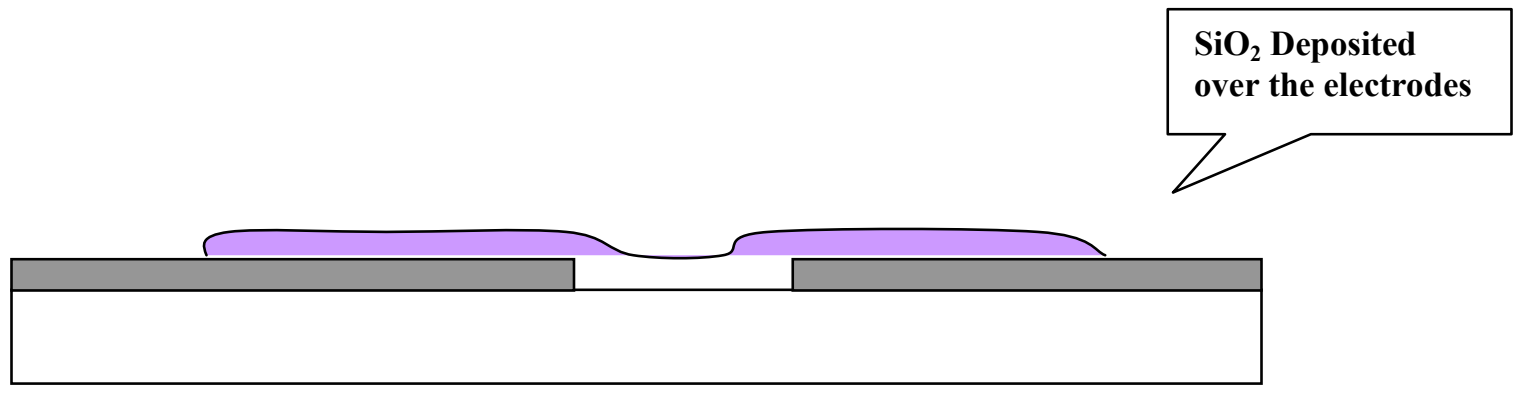

B.6. Bonding of wires to the Chrome slides: Electrical wires were stuck onto the chrome slides using insulating tape. This was to allow the wire to be moving freely and also for simplicity purposes. The chrome slide with the electrical wires looks like the figure below.

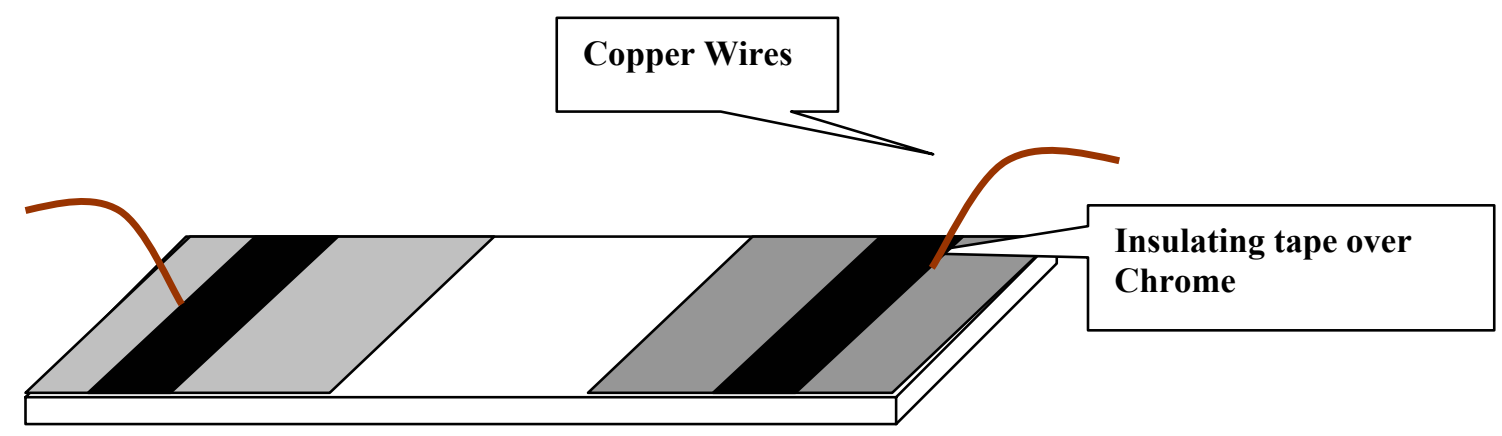

B.7. Wet Etching of Metals: The metal slide after the development process is subjected to wet etching to remove metals. It is an isotropic process. The gold and chrome unprotected by the photoresist is removed during this process. The gold is etched away first by immersing the slide into the gold etch solution. The gold etchant that was used in this process was Transene GE-8111. The slide is placed in a slide holder and then immersed into the etchant for around $2 \frac{1}{2}$ minutes. After the gold has been removed the 
slide is rinsed in DI water to dissolve the residual etchant on the slide. After the gold etching the slide is subjected to a chrome etchant. The slide is while in the slide holder is immersed in the chrome etchant. The chrome etchant used is Transene-Chrome Mask Etchant. The etch time is around 8 minutes.

The etch time of the metal depends on the etch rate of the etchant. The details of it are mentioned in the table. After removing the slide from the etchant it is rinsed in 5\% Sulfuric acid $\left(\mathrm{H}_{2} \mathrm{SO}_{4}\right)$ for 5 minutes and then rinsed in DI water for 5 minutes. The rinsing in $\mathrm{H}_{2} \mathrm{SO}_{4}$ is to prevent frosting of the slide after chrome etch.

After rinsing in DI water, the slide is rinsed in Acetone and Methanol for removal of photoresist from the slide. The rinse time is 5 minutes. The cross-section of the slide looks like the figure below.

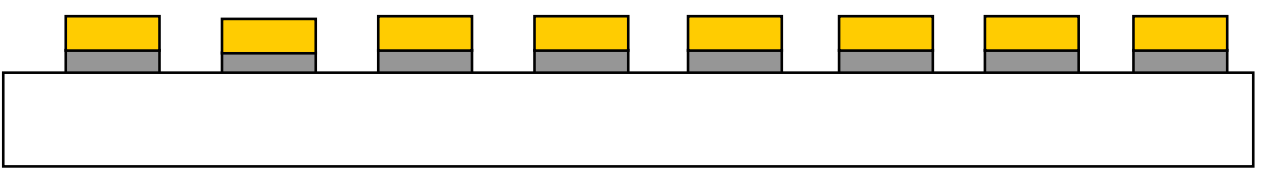

B.8. CVD deposition of $\mathrm{SiO}_{2}$ : Insulating layer of $\mathrm{SiO}_{2}$ was deposited over the etched slide. This process was carried out in a Plasma Enhanced Chemical Vapor Deposition (PECVD) machine. The thickness of $\mathrm{SiO}_{2}$ deposited is approximately $1 \mu \mathrm{m}$. A recipe for deposition of the insulating layer is followed. The PECVD is a computer operated machine and the recipes are stored in the PECVD machine software. After the deposition

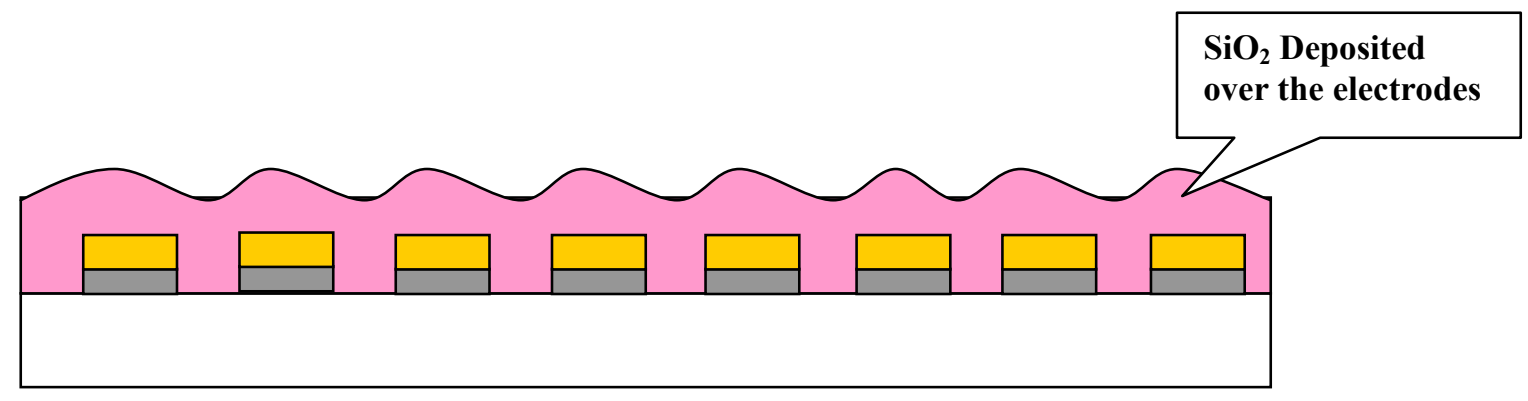


the cross-section looks like the figure below. Care was taken to see that the contact pads remained covered and that there was no deposition of $\mathrm{SiO}_{2}$ on them.

B.9. Bonding of wires: The electrical contacts to the microelectrode slides were done with silver epoxy paste. The silver epoxy paste is a conductive paste. The copper wires used for the wiring were dipped into silver epoxy paste, and then stuck onto the contact pads of the slide. The silver epoxy was bought from Transene; it was of the type A. After sticking the wires onto the slide the slide was cured in an oven at a temperature of $150^{\circ} \mathrm{C}$ for $1 / 2$ hour and at $200^{\circ} \mathrm{C}$ for 1 hour. Alternatively the curing could be carried out at a temperature of $150^{\circ} \mathrm{C}$ for $2 \mathrm{hrs}$. The curing causes the epoxy bond to solidify.

After curing the wires are taped to the end of the slide to prevent the bond from coming loose because of the strain developed in the wires when handling it. The tape used is an insulation tape. 


\section{Appendix C}

Programs for the generation of the 2-D and 3-D plots for Brownian motion BROWNIAN_3D.M SIMULATES A THREE-DIMENSIONAL BROWNIAN MOTION clc,close all,clear all;

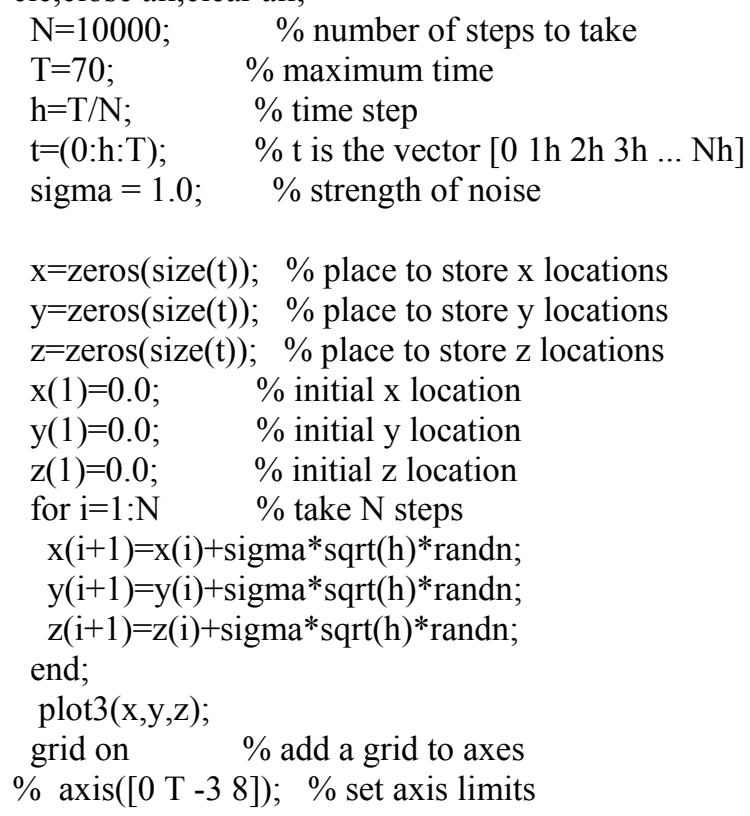

\section{BROWNIAN_2D.M SIMULATES A TWO-DIMENSIONAL BROWNIAN MOTION}

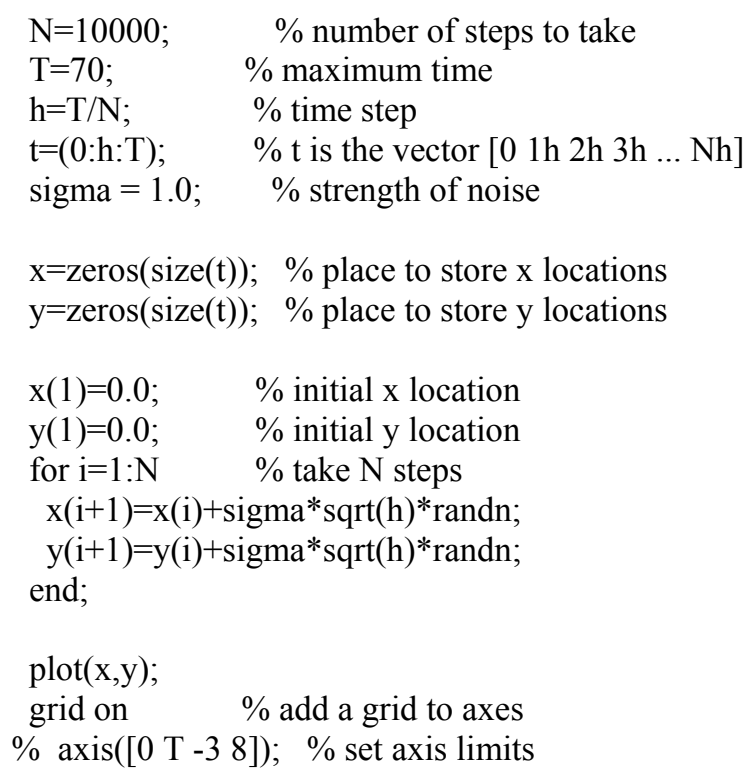

